\title{
MILLIMETER WAVE RADAR OBJECT DETECTION THROUGH FREQUENCY SELECTIVE SURFACES
}

\author{
A Thesis \\ presented to \\ the Faculty of California Polytechnic State University, \\ San Luis Obispo
}

\author{
In Partial Fulfillment \\ of the Requirements for the Degree \\ Master of Science in Electrical Engineering
}

by

Jacob Trevithick

September 2019 
(c) 2019

Jacob Trevithick

ALL RIGHTS RESERVED 


\section{COMMITTEE MEMBERSHIP}

TITLE: Millimeter Wave Radar Object Detection

Through Frequency Selective Surfaces

AUTHOR: Jacob Trevithick

DATE SUBMITTED: September 2019

COMmitTeE CHAIR: Dennis Derickson, Ph.D.

Professor of Electrical Engineering

COMMITTEE MEMBER: Dean Arakaki, Ph.D.

Associate Professor of Electrical Engineering

COMMITTEE MEMBER: Ali Dehghan Banadaki, Ph.D.

Lecturer of Electrical Engineering 


\begin{abstract}
Millimeter Wave Radar Object Detection Through Frequency Selective Surfaces
\end{abstract} Jacob Trevithick

Millimeter wave (mmWave) radar systems are a leading technology in autonomous vehicle object sensing. The radar's ability to detect surrounding objects is critical to its performance. One method of increasing object detection performance is to enhance object visibility. Frequency selective reflectors can increase object visibility. This work examines the performance of a mmWave $77 \mathrm{GHz}$ radar system developed by Texas Instruments in conjunction with frequency selective surfaces. Two bandpass frequency selective surfaces are designed and fabricated using a loaded cross aperture configuration to analyze their application to object detection. The chosen design frequencies are $8 \mathrm{GHz}$ and $79 \mathrm{GHz}$. The frequency selective surfaces are designed and simulated in 3-D electromagnetic simulation software, High Frequency Structure Simulator (HFSS). The frequency selective surfaces are fabricated on $127 \mu m$ thick FR4 dielectric. The $8 \mathrm{GHz}$ frequency selective surface demonstrates bandpass center frequency at $8.12 \mathrm{GHz}$. The $8 \mathrm{GHz}$ and $79 \mathrm{GHz}$ frequency selective surface reflection characteristics are compared to a copper sheets with the same physical cross section as each respective design. Although different testing methodology is used for each design, both frequency selective surfaces demonstrate bandpass characteristics at their respective design frequencies. 


\section{ACKNOWLEDGMENTS}

Thanks to:

- I would like thank my advisor Dr. Derickson for the constant guidance and feedback throughout this thesis project. His insight helped me greatly throughout this process. I'd also like to thank my committee members Dr. Arakaki and Dr. Banadaki. I'd like to thank my friends and family for their encouragement. Finally, I would like to thank my parents for their constant support and love. 


\section{TABLE OF CONTENTS}

LIST OF TABLES . . . . . . . . . . . . . . . . . . . . . . . . . . . . . . . . . . . . . . . . $\ldots$ iii
LIST OF FIGURES
CHAPTER

1 Introduction . . . . . . . . . . . . . . . . . . 1

1.1 Autonomous Vehicles . . . . . . . . . . . . . . . . . 1

1.2 Frequency Selective Surfaces . . . . . . . . . . . . . 2

1.2.1 Frequency Selective Surface Applications . . . . . . . . . . 3

1.3 Development Procedure . . . . . . . . . . . . . . 4

2 Texas Instruments $77 \mathrm{GHz}$ Radar Solution . . . . . . . . . . . . . . 5

2.1 Selection and Evaluation of $77 \mathrm{GHz}$ Testing Platform . . . . . . . 5

2.2 FMCW Radar . . . . . . . . . . . . . . . . . . . 7

2.2.1 Range Measurement ... . . . . . . . . . 8

2.2.2 Velocity Measurement . . . . . . . . . . . . . . . . 10

2.2.3 Arrival Angle . . . . . . . . . . . . . . . . . 12

2.3 Data Capture Device . . . . . . . . . . . . . . . 13

2.4 Characterization . . . . . . . . . . . . . . 15

3 Frequency Selective Surfaces . . . . . . . . . . . . . . . . 17

3.1 Basic Operating Principles . . . . . . . . . . . . . . 17

3.2 FSS Element Types . . . . . . . . . . . . . . . . . . . . 19

3.3 Resonant Frequency . . . . . . . . . . . . . . . . . . . . . . 19

3.4 Dielectric Substrate Effects . . . . . . . . . . . . . . . . 20

3.5 Loaded vs. Unloaded . . . . . . . . . . . . . . . . . . 21

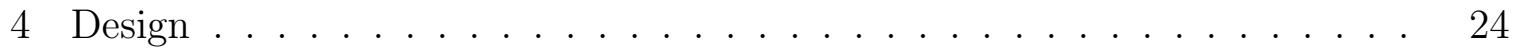

4.1 Loaded Cross Aperture Configuration . . . . . . . . . . . . . . . 24

4.2 HFSS Simulations . . . . . . . . . . . . . . . 25

$4.379 \mathrm{GHz}$ design . . . . . . . . . . . . . . . . . 26

4.3.1 Cross Dipole and Cross Aperture . . . . . . . . . . . . 27

4.3.2 Dielectric Substrate Effects . . . . . . . . . . . . . . 29 
4.3.3 Inter-element Spacing Analysis . . . . . . . . . . . . . . . 30

4.3.4 Tolerance Analysis . . . . . . . . . . . . . . . . . . . . . 31

$4.48 \mathrm{GHz}$ design . . . . . . . . . . . . . . . . . . . . 32

4.5 Fabrication ......................... 34

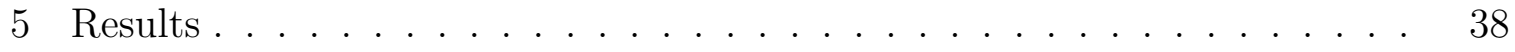

$5.18 \mathrm{GHz} \mathrm{FSS} \ldots \ldots \ldots \ldots$

5.1.1 8 GHz FSS Testing Methodology . . . . . . . . . . 38

5.1.2 Reflection Characteristics . . . . . . . . . . . . . 39

5.1.3 Transmission Characteristics . . . . . . . . . . . . . . . 43

$5.279 \mathrm{GHz}$ FSS . . . . . . . . . . . . . . . . 46

5.2.1 mmWave Methodology . . . . . . . . . . . 46

5.2.2 Reflection Characteristics . . . . . . . . . . . 46

6 Conclusions . . . . . . . . . . . . . . . . . . . . . . . . . . . 49

7 Future Work . . . . . . . . . . . . . . . . . . . . 51

BIBLIOGRAPHY . . . . . . . . . . . . . . . 52 


\section{LIST OF TABLES}

Table

Page

2.1 IWR1642 Radar Field of View Specifications and Measured Results

4.1 Simulated first resonance strength indicator, $8 \mathrm{GHz}$ FSS and $79 \mathrm{GHz}$ FSS ............................ 34

5.1 1D FFT amplitude peaks for various surfaces at $58.59 \mathrm{~cm}$ from the radar . . . . . . . . . . . . . . . . . . . . . . 48

6.1 Simulated and measured resonance frequency at various angles, $8 \mathrm{GHz}$ FSS . . . . . . . . . . . . . . . . . . . 49 


\section{LIST OF FIGURES}

Figure

1.1 Basic bandpass FSS operation diagram at resonant frequency (a), ideal bandpass FSS transmission (b) and reflection curves (c). Unit cells resonant at Stimulus wave frequency, minimal reflections. . . .

2.1 AWR/IWR1642 Evaluation Module (EVM). [4] . . . . . . . . . 6

2.2 Functional Block Diagram AWR12xx, AWR14xx(green), AWR16xx(blue) chipsets. Colored labels indicate included functionality [3]. . . . . . 6

2.3 Chirp signal frequency vs. time, slope $\mathrm{S}=100 \mathrm{MHz} / \mu s$, bandwidth B, and Chirp Period $T_{c}[3] . \ldots \ldots \ldots$. . . . . . . . 7

2.4 Basic FMCW radar block diagram [3]. . . . . . . . . . . . . . 7

2.5 Mixer operation graphical representation, TX chirp (blue), RX chirp (red), and resulting IF signal (green). [3] . . . . . . . . . . . . . 9

2.6 Example chirp frame, $\mathrm{N}$ equally spaced chirp signals. . . . . . . . 11

$2.7 \Delta d$ between two RX antennas from one object $[3] \ldots \ldots$. . . . . . 12

2.8 DCA1000EVM raw ADC data capture card. . . . . . . . . . . 14

2.9 IWR1642 and DCA1000 connection. Device mounted to anechoic chamber test fixture. . . . . . . . . . . . . . . . . . 14

2.10 IWR1642 (red) and DCA1000 (green) setup in the anechoic chamber. Boards mounted to anechoic chamber test fixture. . . . . . . . . . . 15

2.11 Optical Corner Reflector (a), $6 \mathrm{~cm}$ x $6 \mathrm{~cm}$ Copper Sheet (b), Standard Bicycle Reflector(c), and No Testing Object(d) Reflection Measurements. . . . . . . . . . . . . . . . . .

3.1 Typical aperture a) and patch c) FSS, and their typical transmission characteristics b) and d), respectively. Interelement Spacing, $D_{x}$ and $D_{y}[14] \ldots \ldots \ldots \ldots$. . . . . . . . . . . . . . . . . . . . . .

3.2 Typical FSS element type groups (taken from [8], chapter 2, pg. 28) 20

3.3 FSS immersed in infinite dielectric, FSS with no dielectric substrate, FSS with finite thickness dielectric substrate on both sides, and FSS with finite dielectric substrate on one side example transmission/re-

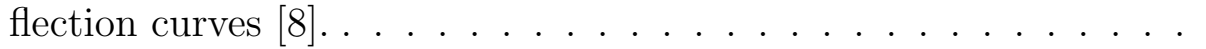


3.4 Unloaded and Loaded square aperture FSS, their equivalent circuits, and their respective transmission curves. Unloaded FSS shows lesser magnitude resonance compared to loaded FSS [8]. . . . . . . . . .

4.1 Cross dipole (a), cross aperture (b), and loaded cross aperture (c) FSS unit cell configurations. Conducting copper surface (orange), dielectric substrate (green). . . . . . . . . . . . . . .

4.2 HFSS Model, FSS Floquet port simulation (a) and Master/Slave boundary $(\mathrm{b}) \ldots \ldots \ldots \ldots$. . . . . . . . . . . . . 26

4.3 $79 \mathrm{GHz}$ FSS unit cell dimensions, $127 \mu m$ (5 mil) thick FR4 (green), 1 oz. copper(orange). . . . . . . . . . . . . . . .

4.4 $79 \mathrm{GHz}$ FSS Reflection (red) and Transmission (purple) HFSS simulations, incident plane wave propagation direction perpendicular to FSS, $127 \mu m$ (5 mil) thick FR4.

4.5 $79 \mathrm{GHz}$ FSS simulated resonance versus frequency at various incident angles, $127 \mu m$ (5 mil) thick FR4. . . . . . . . . . . . . . . 28

4.6 $79 \mathrm{GHz}$ FSS cross dipole HFSS simulation, Figure 4.1a configuration, $S_{21}$ (purple), $S_{11}$ (red), $127 \mu m$ (5 mil) thick FR4. . . . . . . . . 28

4.7 $79 \mathrm{GHz}$ FSS cross aperture HFSS simulation, Figure 4.1b configuration, $S_{21}$ (purple), $S_{11}$ (red), $127 \mu m$ (5 mil) thick FR4. . . . . . . 29

4.8 $79 \mathrm{GHz}$ FSS loaded cross aperture configuration, dielectric substrate removed, $S_{21}$ (purple), $S_{11}$ (red). . . . . . . . . . . . . . . .

4.9 $79 \mathrm{GHz}$ FSS loaded cross aperture configuration $S_{11}$ reflection curves, dielectric substrate thickness, H_diel, swept logarithmically $1.27 \mu \mathrm{m}$ - $1270 \mu m \ldots \ldots \ldots \ldots \ldots . \ldots \ldots . \ldots \ldots$

4.10 $79 \mathrm{GHz}$ FSS loaded cross aperture configuration, Duroid substrate $\left(\epsilon_{r}=2.2\right), S_{21}$ (purple), $S_{11}$ (red) . . . . . . . . . . .

4.11 $79 \mathrm{GHz}$ FSS loaded cross aperture configuration $S_{11}$ reflection curves,

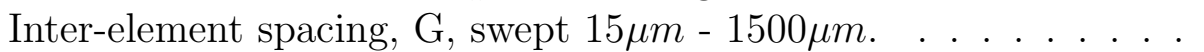

4.12 $79 \mathrm{GHz}$ FSS loaded cross aperture configuration dimensions, inner dipole and aperture width, length, and gap size increased by $10 \%$. .

4.13 $79 \mathrm{GHz}$ FSS loaded cross aperture configuration HFSS simulation, inner dipole and aperture width, length, and gap size increased by $10 \%, S_{21}$ (purple), $S_{11}$ (red). . . . . . . . . . . .

4.14 $79 \mathrm{GHz}$ FSS loaded cross aperture configuration, inner dipole and aperture width, length, and gap size decreased by $10 \%, S_{21}$ (purple), $S_{11}$ (red). . . . . . . . . . . . . . . .

$4.158 \mathrm{GHz}$ FSS unit cell dimensions. . . . . . . . . . . . . . . . 33 
4.16 8 GHz FSS HFSS simulated $S_{11}$ reflection (red) and $S_{21}$ transmission (purple). . . . . . . . . . . . . . . . . . . 34

4.17 $8 \mathrm{GHz}$ FSS simulated $S_{11}$ reflection curves, incident angle sweep, \$theta_scan, $0^{\circ}-45^{\circ}, 15^{\circ}$ steps. . . . . . . . . . . . 35

4.18 Gerber File layouts . . . . . . . . . . . . . . . . 36

4.19 Fabricated FSS designs. . . . . . . . . . . . . . . . 37

5.1 $8 \mathrm{GHz}$ FSS experimental setup diagram, Chamber approximately $2 \mathrm{~m} \times 3 \mathrm{~m}$ (not to scale). . . . . . . . . . . . . 38

5.2 $8 \mathrm{GHz}$ FSS experimental setup, straight on view (a), side view (b). $\quad 39$

5.3 Measured $S_{11}$ vs. Frequency, Copper Sheet (blue), $8 \mathrm{GHz}$ FSS (green). 40

$5.48 \mathrm{GHz}$ FSS experimental Setup Diagram depicting reflection and transmission coefficients. . . . . . . . . . . . . . 40

5.5 High level time domain filtering block diagram. . . . . . . . . . 41

5.6 Measured $S_{11}$ reflection measurement vs. distance, Copper Sheet with NO TDR gate filter (orange), Copper Sheet with TDR gate function (blue) . . . . . . . . . . . . . . . . . . 41

5.7 Measured $S_{11}$ response vs. frequency, copper sheet (blue), $8 \mathrm{GHz}$ FSS (green), TDR gating distance $0.4809 \mathrm{~m}-0.6581 \mathrm{~m}$. . . . . . . 42

5.8 Measured $S_{11}$ response vs. frequency, Theta $=0^{\circ}-45^{\circ}$, no TDR

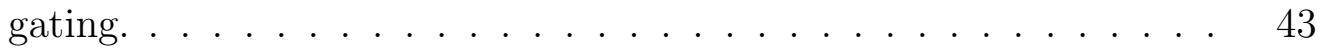

5.9 Measured $S_{11}$ response vs. frequency, Theta $=0^{\circ}-45^{\circ}$, TDR gating used: $45^{\circ}=0.252 m-0.5928 m, 30^{\circ}=0.252 m-0.6015 m$, $15^{\circ}=0.5497 m-0.6279 m, 0^{\circ}=0.4809 m-0.6581 m . \ldots$.

$5.1010 \mathrm{~cm} \times 10 \mathrm{~cm}$ Copper sheet, and No Sheet measured $S_{21}$ vs. frequency. . . . . . . . . . . . . . . . . . . 44 44

$5.118 \mathrm{GHz}$ FSS, and No Sheet measured $S_{21}$ vs. frequency. . . . . . 44

5.12 Calculated $S_{21}$ passband, $8 \mathrm{GHz}$ FSS, $f=7.60-9 \mathrm{GHz} \ldots \ldots 45$

$5.138 \mathrm{GHz}$ FSS measured $S_{21}$ vs. frequency at various theta angles. . . 45

5.14 IWR1642 and DCA1000 experimental setup. . . . . . . . . 46

5.15 Measured Reflection diagram, $79 \mathrm{GHz}$ FSS. . . . . . . . . 47

5.16 Measured Reflection diagram, $6 \mathrm{~cm} \mathrm{x} 6 \mathrm{~cm}$ copper sheet. . . . . . 47 
Chapter 1

\section{INTRODUCTION}

\subsection{Autonomous Vehicles}

Several automotive industry leaders are implementing autonomous features into their vehicle products. These features include autopilot modes such as, cruise control, lane assist, and automatic braking. Fully autonomous modes without driver interaction are currently being tested in standard vehicles.

Automotive vehicle autonomy is defined at six levels [7]:

- Level 0: No autonomy; the driver has full control of all vehicle tasks.

- Level 1: Some driver assistance features are included, such as, cruise control.

- Level 2: Some automated functions, such as acceleration and steering. Driver must remain ready to perform any task at all times.

- Level 3: Vehicle may be operated without driver interaction. However, driver must remain ready to take over at all times.

- Level 4: Vehicle is fully autonomous under certain circumstances and does not require driver interaction. Driver may control the vehicle at any point.

- Level 5: Vehicle is fully autonomous under all conditions. Driver interaction is not necessary, but the driver may still take control if desired.

Object sensing is critical to autonomous vehicle systems. Accurate object detection and classification allows autonomous systems to accurately detect nearby vehicles, pedestrians, and other obstacles. The two leading technologies in object sensing 
are Light detection and ranging (LIDAR) and $77 \mathrm{GHz}$ radio detection and ranging (RADAR). LIDAR sends laser light to illuminate objects in the field of view and measures the reflected light. The objects position is determined through return time and wavelength. Radar is based on the same principles except it uses electromagnetic (EM) waves. These two systems are driving research and development within automotive companies. However, $77 \mathrm{GHz}$ radar is lower cost, less computationally intensive, and more resilience to adverse weather conditions. These factors have caused it prevalence in the automotive sensing market.

There are two methods for improving object detection accuracy: increasing the sensor effectiveness, and increasing object visibility. This work examines the later in application to $77 \mathrm{GHz}$ radar. Increasing the object's reflection properties in the sensor's frequency range will increase the reflected EM power from the object. One possible solution is to utilize a device that is highly reflective and design a surface that modifies the bandpass bandwidth to the sensor frequency range. A frequency selective surface is a structure that demonstrates filtering properties and is examined in this application.

\section{$1.2 \quad$ Frequency Selective Surfaces}

Frequency selective surfaces (FSS) are periodic structures that demonstrate filtering properties on incident electromagnetic waves. These surfaces include repeating unit cells with resonant properties at a particular design frequency. They can be designed as high pass, low pass, band pass, or band stop filters. FSSs are active or passive filters; with or without external excitation. Figure 1.1 below shows a bandpass FSS's diagram with its typical transmission and reflection curves. 


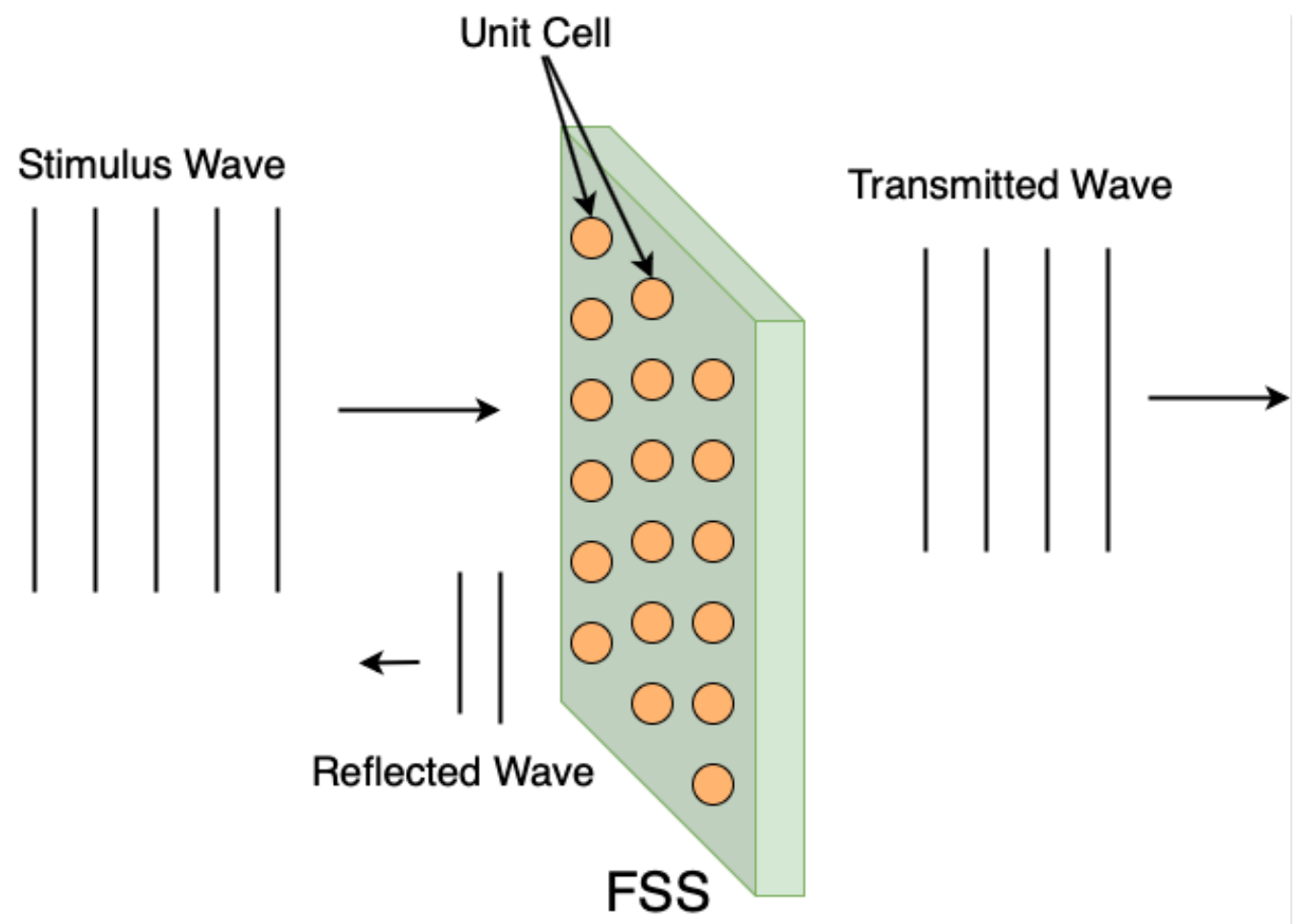

(a)

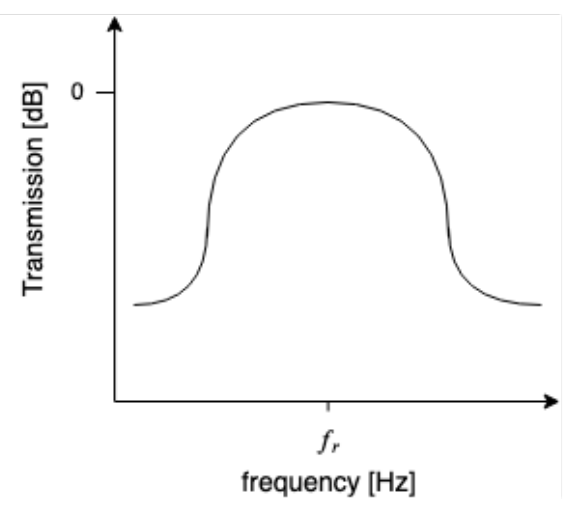

(b)

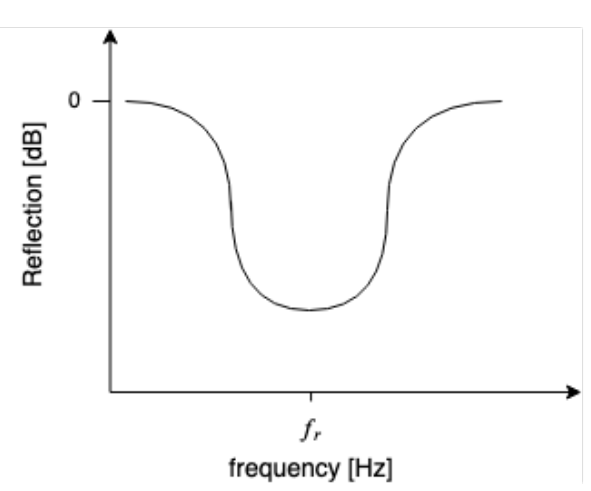

(c)

Figure 1.1: Basic bandpass FSS operation diagram at resonant frequency (a), ideal bandpass FSS transmission (b) and reflection curves (c). Unit cells resonant at Stimulus wave frequency, minimal reflections.

\subsubsection{Frequency Selective Surface Applications}

Numerous FSS commercial and military applications exist. Many commercial microwave ovens utilize frequency selective surfaces to reflect $2.45 \mathrm{GHz}$ microwaves and pass visible light [14]. Microwave oven door windows include an FSS screen of periodic 
metallic holes. FSS is implemented in textiles to create frequency selective fabrics for applications in clothing based electronic systems and sensors [12]. These sensors FSS microwave absorbers have been investigated using resistive patches and $\frac{\lambda}{4}$ absorbing dielectrics [5]. This is also applied to mmWave frequency ranges 75-110 GHz [10]. Military vehicle radar cross section is reduced using active FSSs [2]. Multi-layer FSS that are typically used to reduce radar cross section [8]. FSS is used in high performance microstrip antennas [9]. Dual band FSS (24 GHz and $77 \mathrm{GHz})$ is applied to radar antennas [11].

\subsection{Development Procedure}

A functional $77 \mathrm{GHz}$ radar system is utilized to analyze FSS performance. The $77 \mathrm{GHz}$ radar system operation is characterized and compared with manufacturer specifications. An FSS design is chosen and the reflection and transmission properties are simulated in High Frequency Structure Simulator (HFSS). FSS resonant frequencies $79 \mathrm{GHz}$ and $8 \mathrm{GHz}$ are implemented. The $8 \mathrm{GHz}$ FSS is developed to allow transmission characteristic analysis with a Vector Network Analyzer that has a frequency range capable of testing the FSS. A prototype design is developed and fabricated at both resonant frequencies using external PCB fabricator, Bay Area Circuits. The $79 \mathrm{GHz}$ design frequency is chosen as the center $77 \mathrm{GHz}$ radar's chirp center frequency. This creates maximal transmission through the FSS at the radar's operation frequency. The $77 \mathrm{GHz}$ radar system is used to measure the $79 \mathrm{GHz}$ FSS reflection characteristics. The $8 \mathrm{GHz}$ FSS transmission and reflection characteristics are measured using an Anritsu MS4624B Vector Network Analyzer. 
Chapter 2

TEXAS INSTRUMENTS 77GHZ RADAR SOLUTION

\subsection{Selection and Evaluation of $77 \mathrm{GHz}$ Testing Platform}

Texas Instruments (TI) has developed a suite of millimeter wave (mmWave) radar systems. It includes the AWR12xx/AWR14xx/AWR16xx and IWR12xx/IWR14xx/IWR16xx radar systems. The AWR and IWR chipsets are functionally identical; the AWR is qualified for automotive applications. The TI IWR1642BOOST Evaluation Module (EV) $77 \mathrm{GHz}$ mmWave radar is the testing device used in this Thesis. Figures 2.1a and $2.1 \mathrm{~b}$ show the front and rear board views, respectively. Figure 2.2 shows the AWR1x system functional high level block diagram. Further system descriptions are found in [3].

The device is a mmWave frequency-modulated continuous wave (FMCW) radar system. The complete radar system contains two transmit (TX) and four receive (RX) antennas, a complete radio frequency (RF) analog front end, digital components such as analog-to-digital converters, a fully integrated digital signal processor (DSP), and a microcontroller (MCU) [6]. Fully integrating these components onto a single chip decreases power consumption and overall system cost. The IWR1642 radar device is designed using complementary metal-oxide semiconductor (CMOS). The radar device utilizes FMCW to measure position, velocity, and arrival angle, discussed further in later sections. 


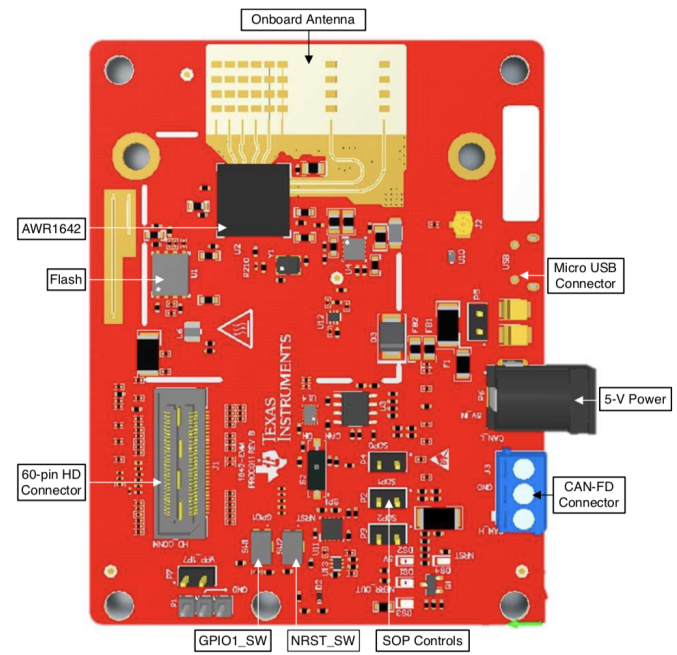

(a) Front

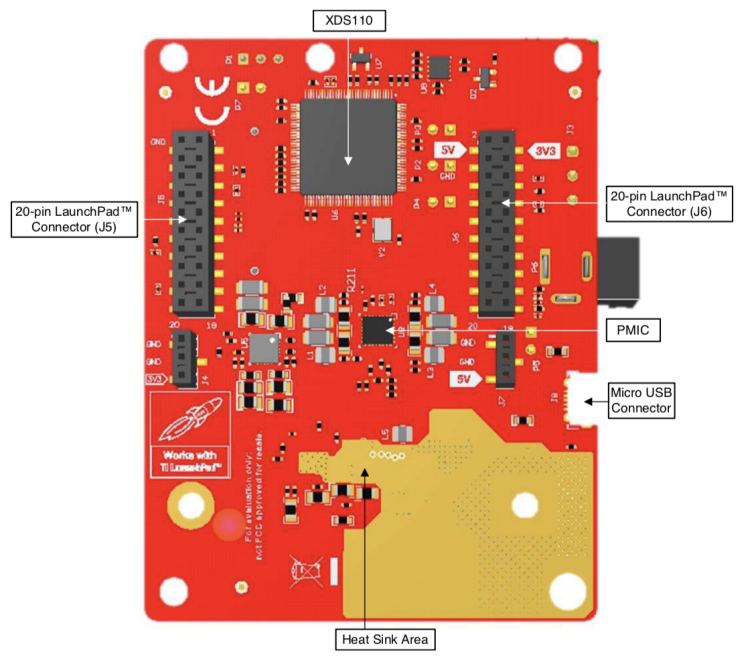

(b) Rear

Figure 2.1: AWR/IWR1642 Evaluation Module (EVM). [4].

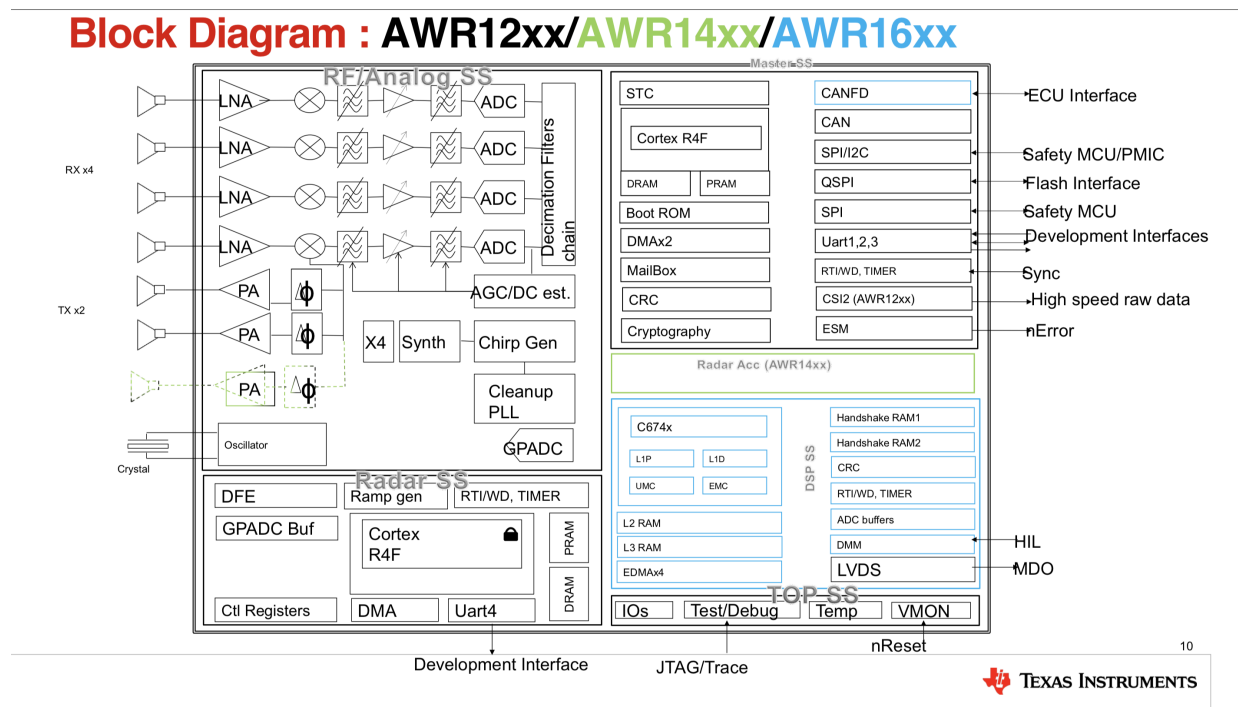

Figure 2.2: Functional Block Diagram AWR12xx, AWR14xx(green), $\operatorname{AWR} 16 \times x($ blue $)$ chipsets. Colored labels indicate included functionality [3]. 


\subsection{FMCW Radar}

Radar systems transmit electromagnetic signals which are reflected by objects. An FMCW radars signal frequency increases linearly with time; a chirp signal. Figure 2.3 below shows the IWR1642 chirp signal with frequency as a function of time.

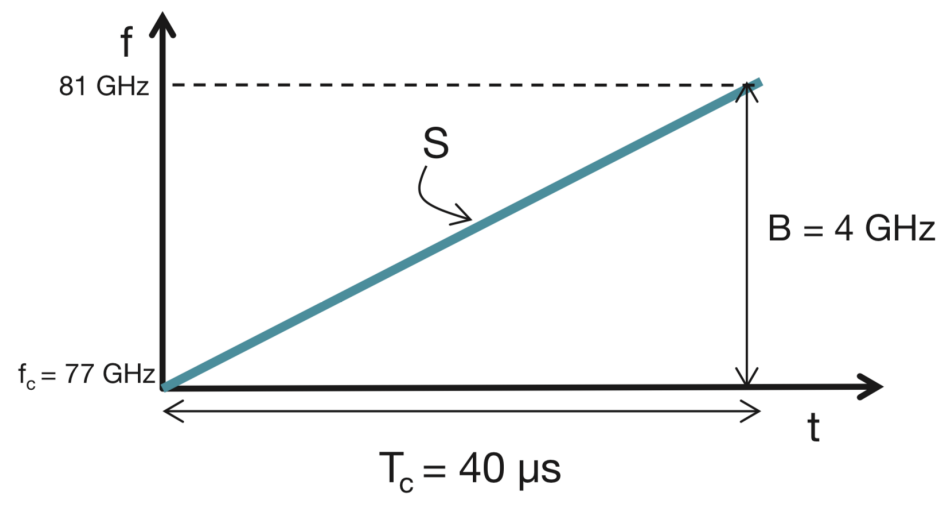

Figure 2.3: Chirp signal frequency vs. time, slope $\mathrm{S}=100 \mathrm{MHz} / \mu \mathrm{s}$, bandwidth B, and Chirp Period $T_{c}[3]$.

The transmitted chirp signal is reflected from objects, detected by radar receive antennas, then mixed with the original chirp signal. The resultant intermediate frequency (IF) signal is converted to the objects range. Figure 2.4 below depicts the basic FMCW block diagram.

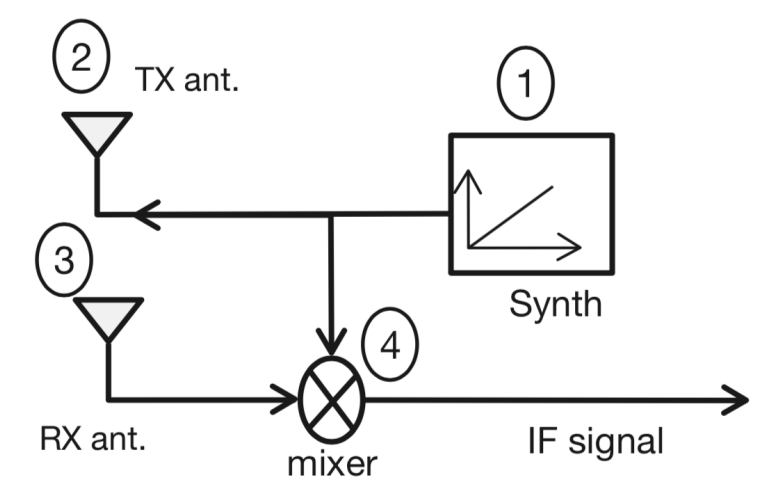

Figure 2.4: Basic FMCW radar block diagram [3]. 


\subsubsection{Range Measurement}

The IF signal is the difference between the TX and RX chirp signals. The input signals to the mixer are defined as $c_{1}$ and $c_{2}$ below.

$$
\begin{aligned}
& c_{1}=\sin \left(\omega_{1} t+\phi_{1}\right) \\
& c_{2}=\sin \left(\omega_{2} t+\phi_{2}\right)
\end{aligned}
$$

After initial analog filtering, the mixer output signal is,

$$
c_{I F}=\sin \left[\left(\omega_{1}-\omega_{2}\right) t+\left(\phi_{1}-\phi_{2}\right)\right]
$$

Figure 2.5 graphically depicts a TX chirp and RX chirp and the resulting IF signal. $\tau$ represents the time delay between the TX and RX signals, and is defined as,

$$
\tau=\frac{2 d}{c}
$$

where $d$ is the distance to the object and $c$ is the speed of light in a vacuum. $T_{c}$ is the observation window for the TX chirp. The RX chirp must be detected within the observation window to detect the object. The IF frequency is the difference between TX and RX chirp frequencies and remains constant. 


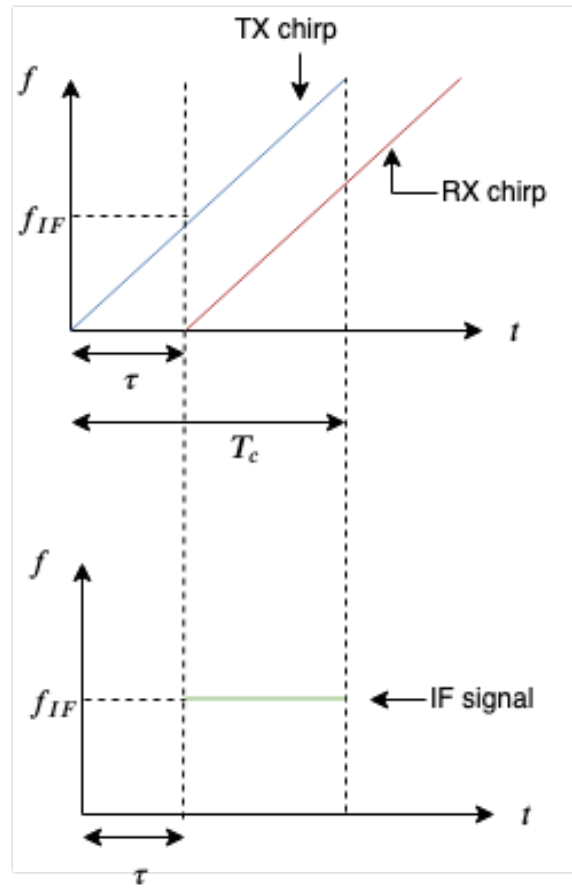

Figure 2.5: Mixer operation graphical representation, TX chirp (blue), RX chirp (red), and resulting IF signal (green). [3]

The IF signal phase is the phase difference between the TX and RX chirps at the IF signal start time.

$$
\phi_{0}=2 \pi f_{c} \tau=\frac{4 \pi d}{\lambda}
$$

The IF signal is therefore defined as,

$$
c_{I F}=A \sin \left(2 \pi f_{0} t+\phi_{0}\right)
$$

where $f_{0}=S \tau$. Multiple object detections leads to a mixer output with multiple frequency components corresponding to each object. To differentiate between objects, a Fourier transform creates frequency peaks corresponding to each object. Frequency differences are converted to object ranges.

Individual object identification, radar resolution, is determined from IF signal duration and chirp signal bandwidth. Two IF signal tones can be resolved in the 
frequency domain if the difference between their respective frequencies satisfies,

$$
\Delta f=\frac{S 2 \Delta d}{c}>\frac{1}{T_{c}}
$$

which is expressed as object separation distance

$$
\Delta d>\frac{c}{2 S T_{c}}=\frac{c}{2 B}
$$

where $B=S T_{c}$ is the chirp bandwidth. Therefore, the radar's range resolution is only dependent on the chirp signal bandwidth.

$$
d_{\text {res }}=\frac{c}{2 B}
$$

The IWR1642 has $\mathrm{B}=4 \mathrm{GHz}$ and therefore, a range resolution, $d_{\text {res }}=3.75 \mathrm{~cm}$.

\subsubsection{Velocity Measurement}

Detected object velocity is determined using two chirps transmitted a time $T_{c}$ apart. After a Fast-Fourier Transform (FFT), the reflected chirps from one object will produce a single difference frequency, but with a phase difference. The measured phase difference between the two frequency peaks corresponds to the object's motion. The phase difference between the two signals is defined as,

$$
\Delta \phi=\frac{4 \pi v T_{c}}{\lambda}
$$

corresponding to,

$$
v=\frac{\lambda \Delta \phi}{4 \pi T_{c}}
$$


However, the velocity will only remain unambiguous if $|\Delta \phi|<\pi$. Therefore, the velocity is restricted to,

$$
v<\frac{\lambda}{4 T_{c}}
$$

The maximum resolvable object velocity is determined by the duration between chirp signals $T_{c}$, and is defined as,

$$
v_{\max }=\frac{\lambda}{4 T_{c}}
$$

However, this two-chirp velocity measurement system cannot measure velocity accurately if multiple objects are detected at two different velocities at the same distance from the radar. The objects will generate reflected chirp signals that produce identical IF signal frequencies, leading to a single frequency peak after the FFT. Therefore, the radar must transmit $\mathrm{N}(\mathrm{N}>2)$ chirps all spaced by $T_{c}$. This chirp set is a chirp frame. Figure 2.6 below illustrates a chirp frame with frame period $T_{f}=N T_{c}$

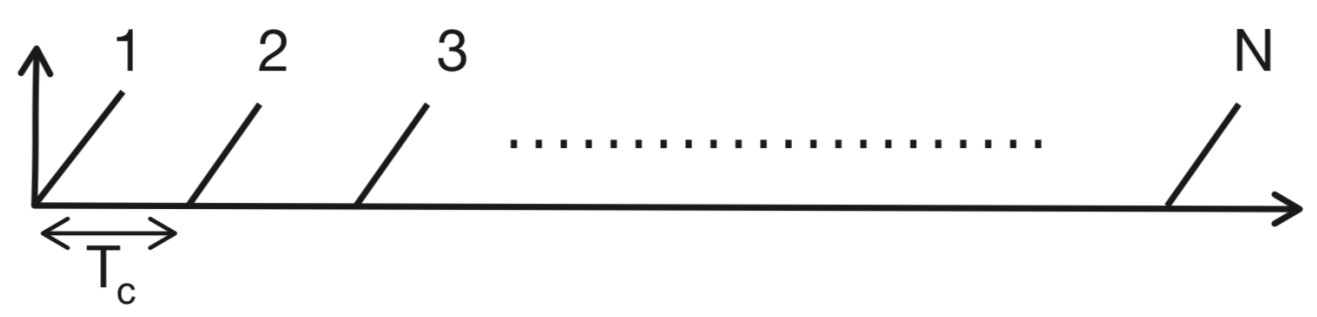

Figure 2.6: Example chirp frame, $\mathrm{N}$ equally spaced chirp signals.

A second FFT is computed to resolve the two objects' velocities. The first and second transforms are Range-FFT and Doppler-FFT. The Range-FFT processes the reflected chirps from the two objects. This corresponds to $\mathrm{N}$ identically located peaks in the frequency domain. However, each object produces a peak with distinct phase values. The Doppler-FFT is performed on the $\mathrm{N}$ phasors to resolve the two objects [3]. This results in two peaks, $\omega_{1}$ and $\omega_{2}$, that correspond to the phase difference 


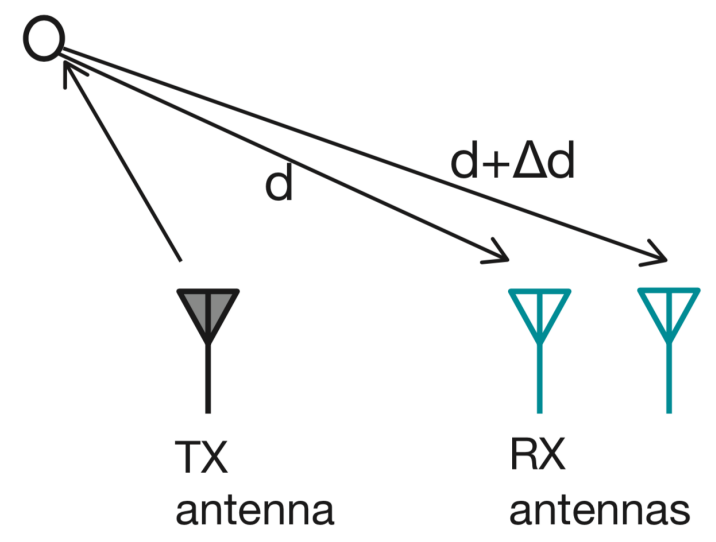

Figure 2.7: $\Delta d$ between two RX antennas from one object [3].

between consecutive chirps for each respective object. The velocity is then calculated as,

$$
v_{1,2}=\frac{\lambda \omega_{1,2}}{4 \pi T_{c}}
$$

Two discrete frequencies are resolvable if $\Delta \omega=\omega_{2}-\omega_{2}>2 \pi / N[\mathrm{rad} /$ sample $]$. Adjusting equation 2.10 to include the chirp frame period results in velocity resolution,

$$
v>v_{r e s}=\frac{\lambda}{2 T_{f}}
$$

Therefore, velocity resolution is inversely proportional to chirp frame period, $T_{f}[3]$.

\subsubsection{Arrival Angle}

The an object's angle with respect to the FMCW radar is the arrival angle. The arrival angle is determined from distance difference $\Delta d$ between two RX antennas. The $\Delta d$ is measured from the resulting phase change between the two peaks in the FFT. Figure 2.7 below illustrates the difference in distance the RX signal travels to arrive at the RX antennas. 
The $\Delta d$ corresponds to the resulting $\Delta \phi$ via,

$$
\Delta \phi=\frac{2 \pi \Delta d}{\lambda}
$$

Assuming the received signal is a plane wave, $\Delta d$ is geometrically approximated,

$$
\Delta d=l \sin (\theta)
$$

where $l$ is the distance between two antennas. Therefore, the arrival angle is computed as,

$$
\theta=\sin ^{-1}\left(\frac{\lambda \Delta \phi}{2 \pi l}\right)
$$

This measurement utilizes the sine function small angle approximation, $\sin (\theta) \approx$ $\theta$. Therefore, the arrival angle estimation is more accurate the closer $\theta$ is to 0 . The maximum angular field of view is defined by the radar's maximum arrival angle measurement [3]. An unambiguous angle measurement requires $|\Delta \phi|<180^{\circ}$. This leads to the maximum field of view defined by the spacing between two antennas is,

$$
\theta_{\max }=\sin ^{-1}\left(\frac{\lambda}{2 l}\right)
$$

Antennas spaced $l=\lambda / 2$ corresponds to a maximum angular field of view $\pm 90^{\circ}$.

\subsection{Data Capture Device}

The DCA1000EVM board is used in conjunction with the IWR1642 to capture realtime radar measurements. The DCA1000 device captures raw ADC data from the radar before FFT processing occurs. The DCA1000 connects to the IWR1642 via a Samtec coaxial micro ribbon cable. The DCA1000 connects via Ethernet cable to the computer. It allows the user to process the data external to the IWR1642 DSP. 
Figure 2.8 below shows the DCA1000EVM capture card board. Figure 2.9 shows the IWR1642 and DCA1000 board connections in the anechoic chamber test fixture.

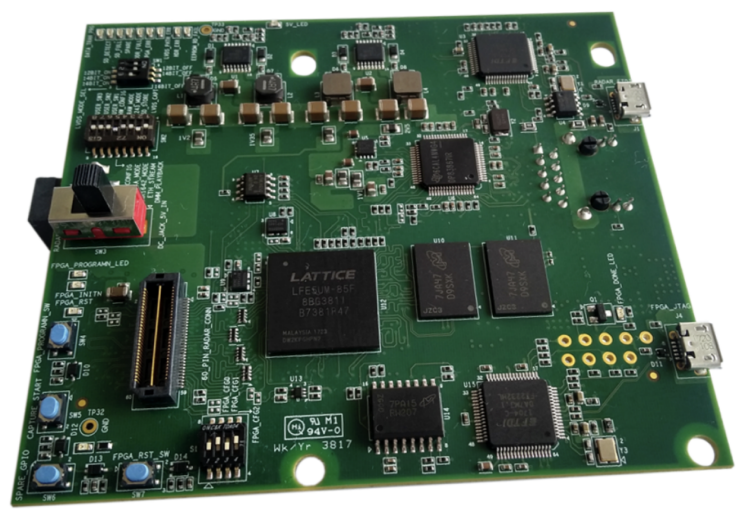

Figure 2.8: DCA1000EVM raw ADC data capture card.

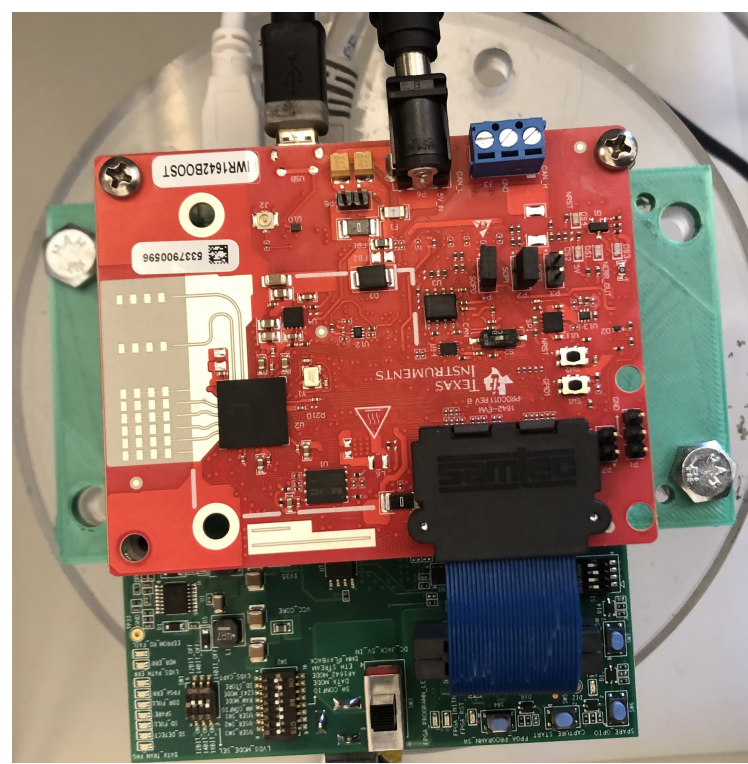

(a)

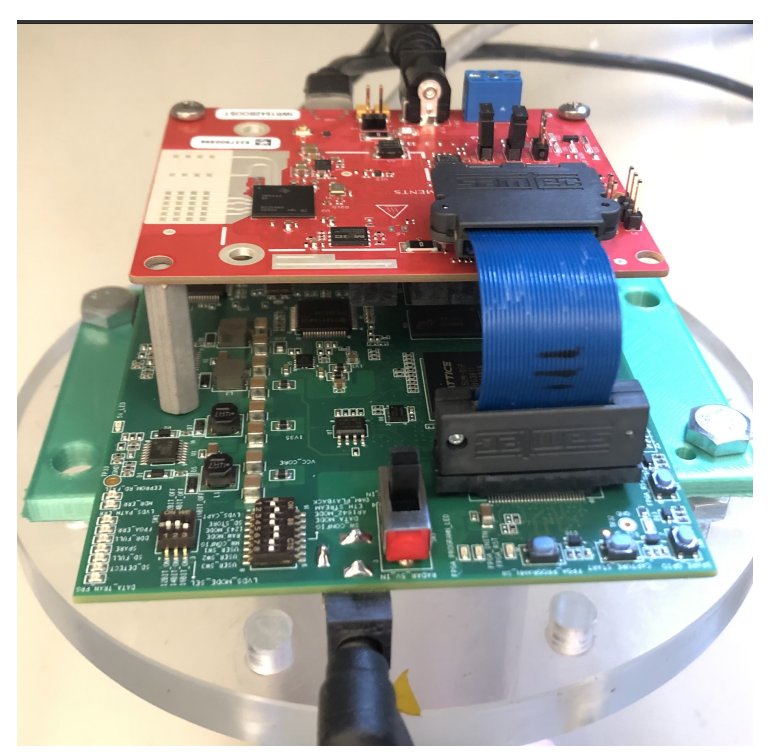

(b)

Figure 2.9: IWR1642 and DCA1000 connection. Device mounted to anechoic chamber test fixture. 


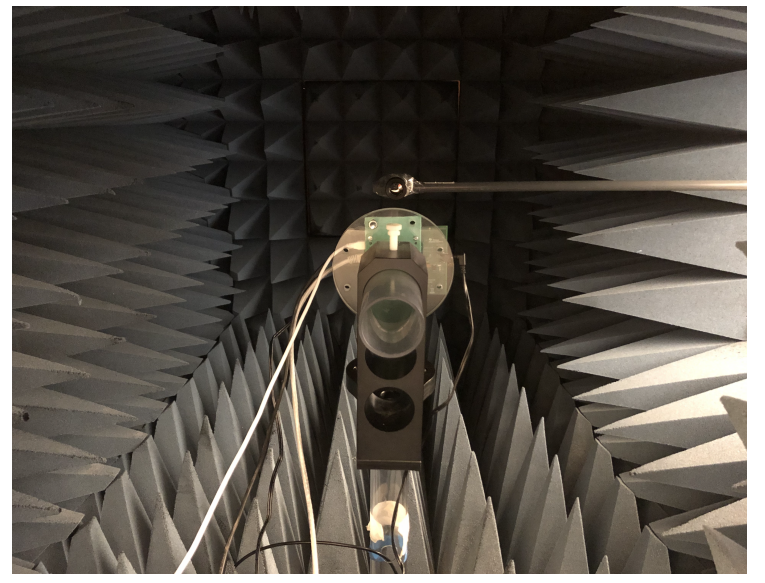

(a)

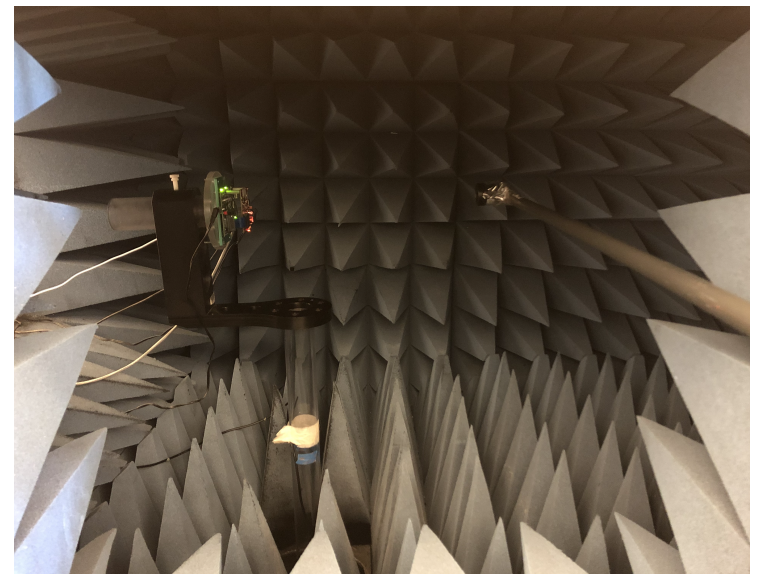

(b)

Figure 2.10: IWR1642 (red) and DCA1000 (green) setup in the anechoic chamber. Boards mounted to anechoic chamber test fixture.

\subsection{Characterization}

The IWR1642 and DCA1000 are tested in the anechoic chamber to measure various objects' reflected power, and the field of view capabilities. Figure 2.10 below shows the experimental setup to determine reflected power and field of view measurements.

IWR1642 board characterization includes reflection measurements on an optical corner cube reflector, a $6 \mathrm{~cm} \mathrm{x} 6 \mathrm{~cm}$ copper sheet and a standard bicycle reflector. Figure 2.11 below shows object amplitude profiles. The vertical axis shows the measured reflection amplitude in decibels with respect to full scale (dBFS).

The IWR1642 field of view specifications and measurements are shown in Table 2.1 below. The measured azimuthal range greater than the given specifications. This is likely due to anechoic testing where noise sources are significantly reduced.

Table 2.1: IWR1642 Radar Field of View Specifications and Measured Results

\begin{tabular}{|c|c|c|}
\hline Specification & Datasheet Value & Measured Value \\
\hline Azimuthal Range & $\approx 120^{\circ}$ & $167.5^{\circ}$ \\
\hline Elevation Range & $\approx 40^{\circ}$ & $37.7^{\circ}$ \\
\hline
\end{tabular}




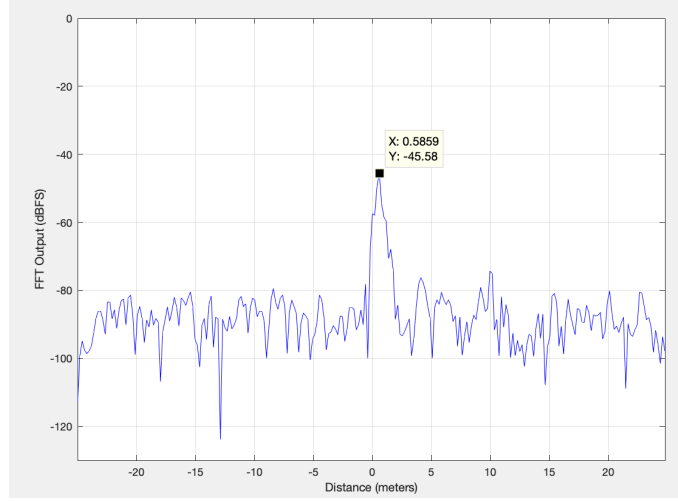

(a) Optical Corner Reflector

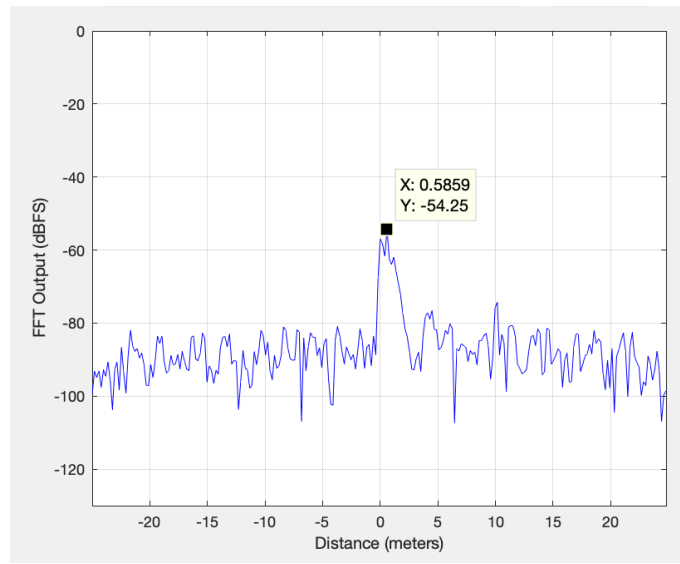

(c) Standard Bicycle Reflector

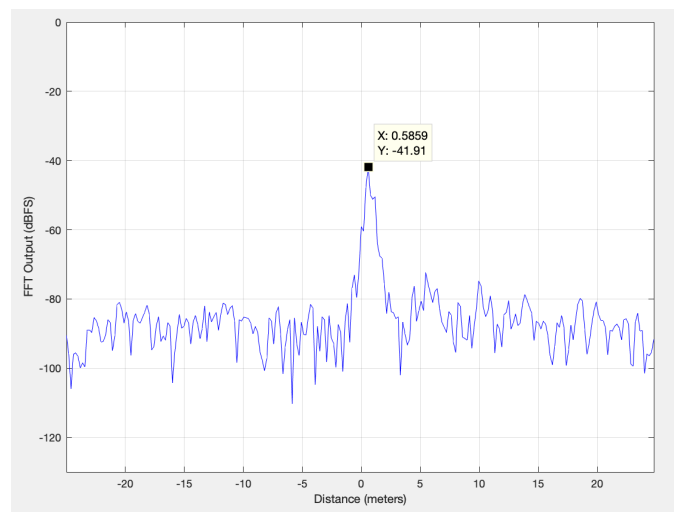

(b) $6 \mathrm{~cm} \times 6 \mathrm{~cm}$ Copper Sheet

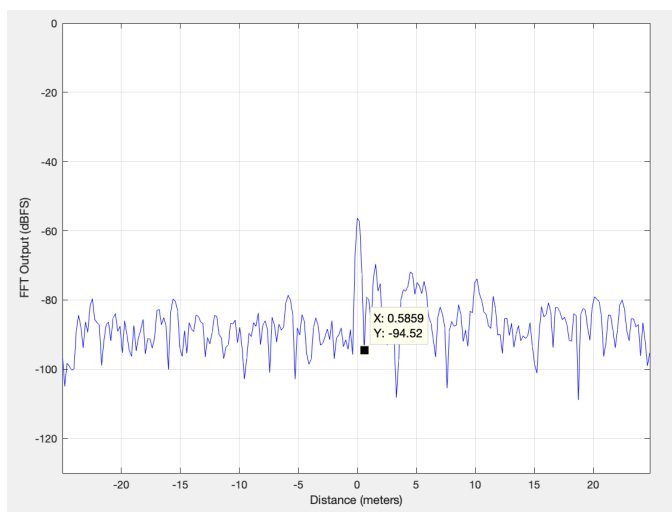

(d) No Testing Object

Figure 2.11: Optical Corner Reflector (a), $6 \mathrm{~cm} \times 6 \mathrm{~cm}$ Copper Sheet (b), Standard Bicycle Reflector(c), and No Testing Object(d) Reflection Measurements. 


\section{Chapter 3}

\section{FREQUENCY SELECTIVE SURFACES}

\subsection{Basic Operating Principles}

A frequency selective surface (FSS) is typically a two-dimensional array of periodic elements [14]. A perfectly conducting aperture FSS resonates and functions as a bandpass filter at the design frequency. The FSS reflects electromagnetic wave frequencies outside the passband. A perfectly conducting patch FSS acts a bandstop filter. The FSS reflects incident electromagnetic waves at the patch resonant frequency. The transmission coefficient, $\tau$, is defined as,

$$
\tau=\frac{E_{t}}{E_{i}}
$$

where $E_{t}$ is the transmitted electromagnetic wave signal amplitude and $E_{i}$ is the incident wave signal amplitude. The reflection coefficient, $\Gamma$, is defined as,

$$
\Gamma=\frac{E_{r}}{E_{i}}
$$

where $E_{r}$ is the reflected wave signal amplitude. Figure 3.1 below depicts the typical patch and aperture structure and their respective transmission curves.

Complimentary arrays, like those shown in Figure 3.1, exhibit reflection curves equal to the transmission curves of one another. However, this is only true in the ideal case where the material is onsidered infinitely thin and perfectly conducting with no dielectric substrate [8]. An electric current is excited in the patch case, and a magnetic current density caused by a voltage distribution across the aperture is excited in the aperture case. 

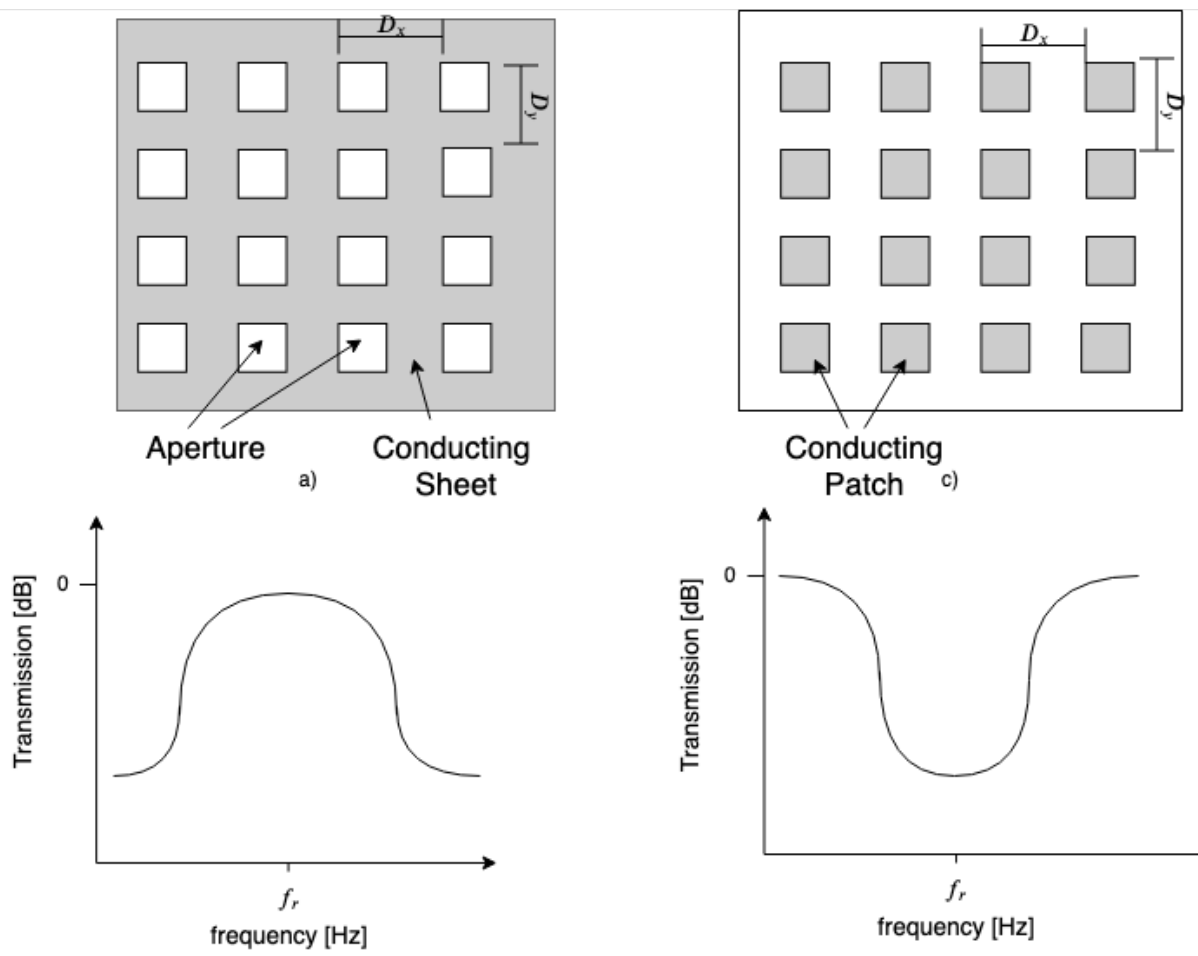

b)

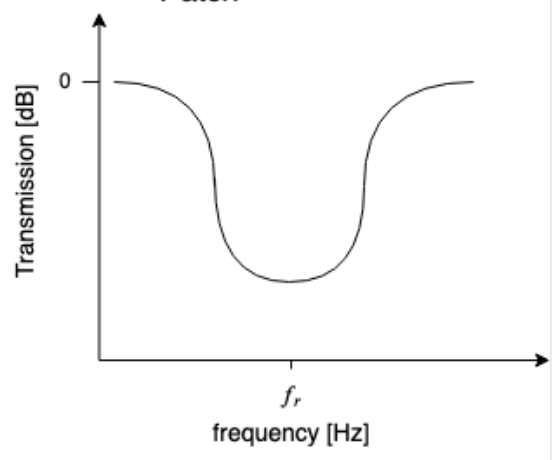

d)

Figure 3.1: Typical aperture a) and patch c) FSS, and their typical transmission characteristics b) and d), respectively. Interelement Spacing, $D_{x}$ and $D_{y}[14]$.

The patch FSS case is considered a capacitive mesh with impedance,

$$
Z_{c}=\frac{1}{j \omega C}
$$

where $\mathrm{C}$ is the effective mesh capacitance and $\omega$ is the incident electromagnetic wave angular frequency. An EM wave with frequency near the element's resonant frequency causes near uniform oscillating current densities in each conducting element. The mesh approximates a continuous conducting sheet and reflects the wave.

The aperture FSS is considered an inductive mesh with impedance,

$$
Z_{L}=j \omega L,
$$


where $\mathrm{L}$ is the effective mesh inductance and $\omega$ is the incident electromagnetic wave angular frequency. EM wave frequencies within the passband conduct through the apertures and the EM wave transmits through the surface. At frequencies outside resonance, surface currents conduct in the inductive mesh reflecting the EM wave. The mesh approximates a continuous conducting sheet.

\subsection{FSS Element Types}

FSS element types include center connected, loop, solid/plate, and combinations [8], see figure 3.2 below. The loop type family demonstrates favorable performance metrics compared to the other types. Element size can be reduced using loading (discussed in section 3.5). Reducing element size also enables decreased interelement spacing. Closer elements decreases the resonant frequency variance with respect to EM wave's incident angle upon the FSS [8].

\subsection{Resonant Frequency}

The FSS resonant frequency is determined from element size and shape, dielectric material and thickness, and interelement spacing. The element circumference is typically a multiple of the resonant wavelength. However, the circumference usually matches the resonant wavelength. This reduces the nulls in the scattering pattern [14]. In the

dipole case, the element is typically $\lambda_{r} / 2$ in length. In the square and circular loop case, depicted in Figure 3.2, each half loop acts as a $\lambda_{r} / 2$ dipole element [14].

FSS resonance bandwidth is inversely proportional to interelement spacing. Increasing the distance between elements by $10 \%$ in one dimension decreases the bandwidth by approximately 10\%. Increasing the distance between elements by $10 \%$ in two dimensions decreases the bandwidth by approximately $20 \%$ [8]. 


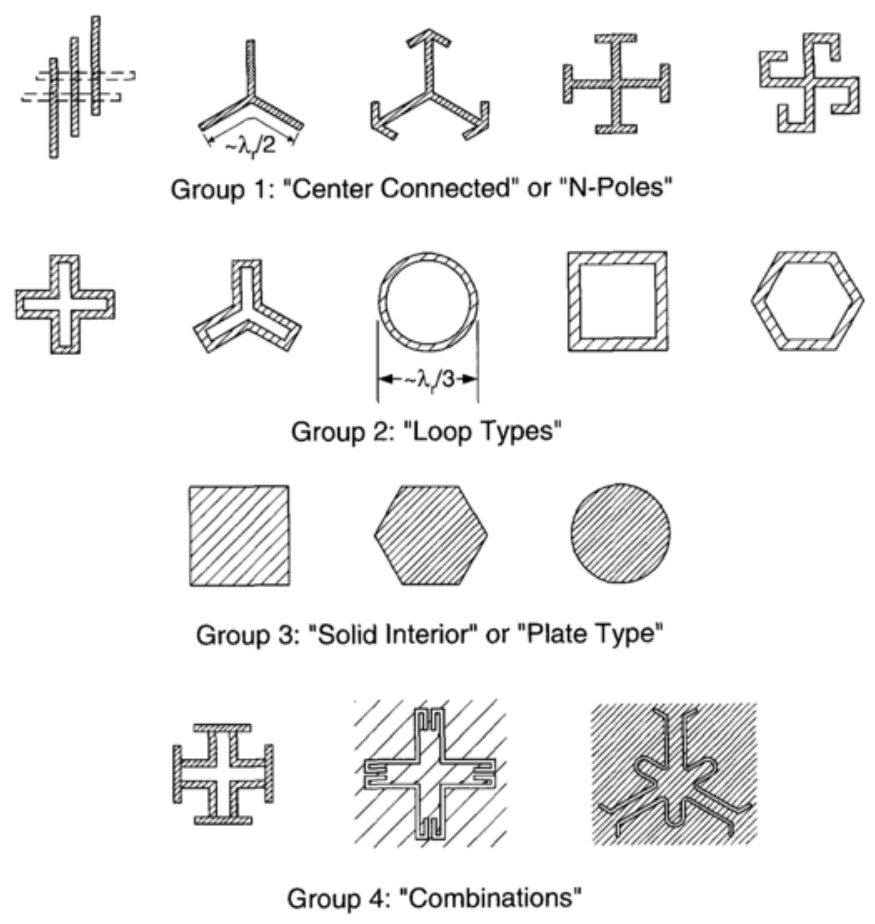

Figure 3.2: Typical FSS element type groups (taken from [8], chapter 2, pg. 28)

\subsection{Dielectric Substrate Effects}

Dielectric substrates provide mechanical strength and control the FSSs resonance magnitude and frequency through the relative dielectric constant $\epsilon_{r}$ and substrate thickness. An FSS with infinitely thick dielectric slabs on both sides resonate at $f_{r} / \sqrt{\epsilon_{r}}[8]$, where $f_{r}$ is the free space resonant frequency. However, reducing the dielectric slabs' thickness to a typical value $\approx 0.05 \lambda_{r}$, the resonant frequency lies between $f_{r} / \sqrt{\epsilon_{r}}$ and $f_{r}$. An FSS with a dielectric slab only on one side will have a minimum resonate frequency at $f_{r} / \sqrt{\left(\epsilon_{r}+1\right) / 2}$ [8]. Figure 3.3 below illustrates the three cases.

Dielectric material loss tangent decreases resonance magnitude. Therefore, loss tangent should be minimized in FSS bandpass filters. However, higher performance materials are typically more costly. 

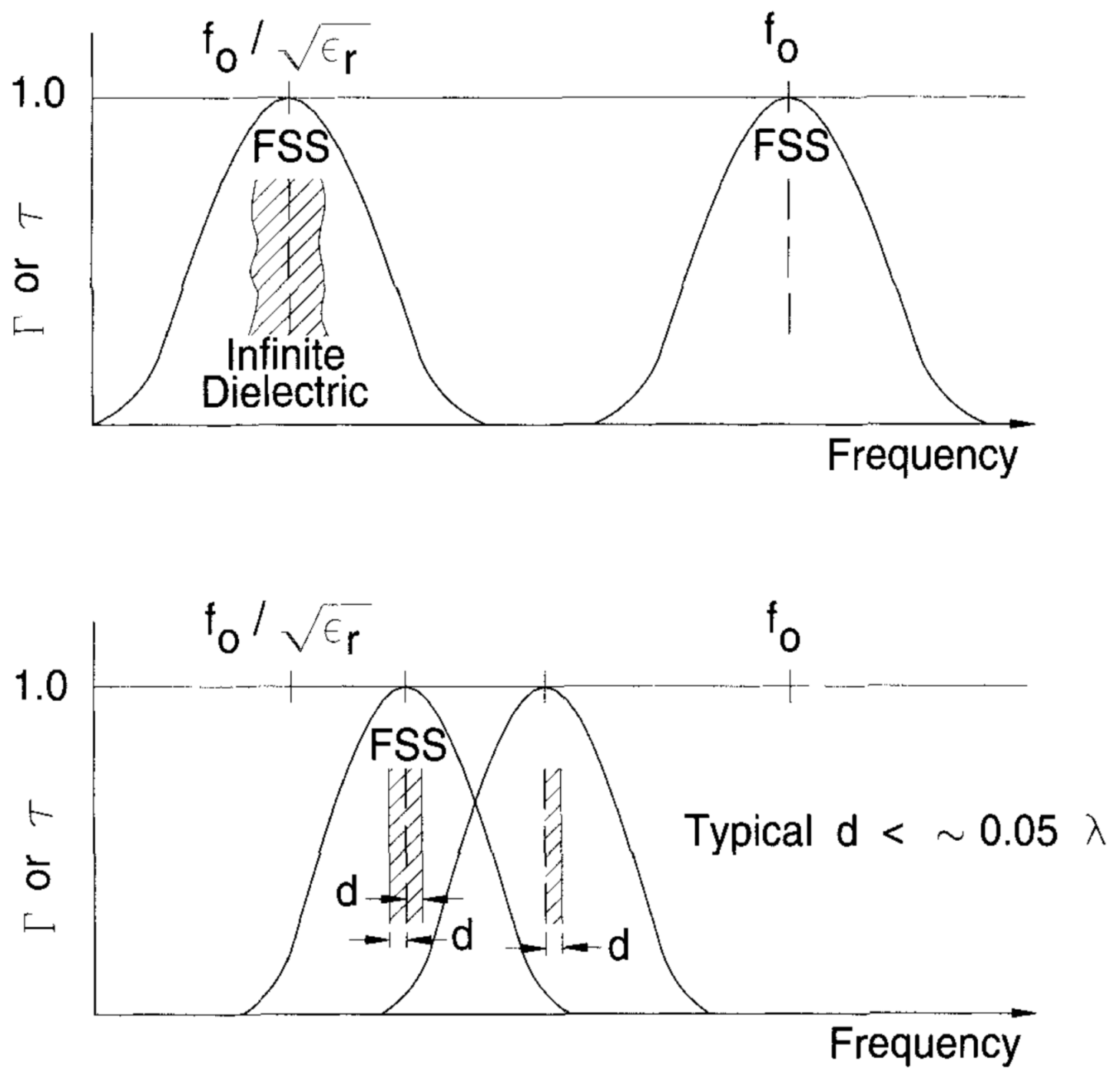

Figure 3.3: FSS immersed in infinite dielectric, FSS with no dielectric substrate, FSS with finite thickness dielectric substrate on both sides, and FSS with finite dielectric substrate on one side example transmission/reflection curves [8].

\subsection{Loaded vs. Unloaded}

Aperture type structures produce a bandpass response. However, this configuration demonstrates non-optimal resonance magnitudes. This is attributed to small capacitance in the equivalent circuit. An LC parallel resonator performance is typically characterized by its $\mathrm{Q}$ value. $\mathrm{Q}$ is the measure of resonator bandwidth compared to 
the center frequency. Generally, Q is defined as,

$$
Q=\frac{f_{r}}{B W}
$$

Furthermore, for a LC parallel resonator Q is defined as,

$$
Q=\omega C R
$$

where $\omega$ is the angular resonant frequency, $\mathrm{C}$ is the equivalent capacitance, and $\mathrm{R}$ is the equivalent circuit resistance. Inserting a loading element into the aperture increases the equivalent capacitance. Therefore, the loading effect increases the $\mathrm{Q}$ of the equivalent circuit resonator. Figure 3.4 demonstrates this phenomena in the square aperture bandpass filter case. 

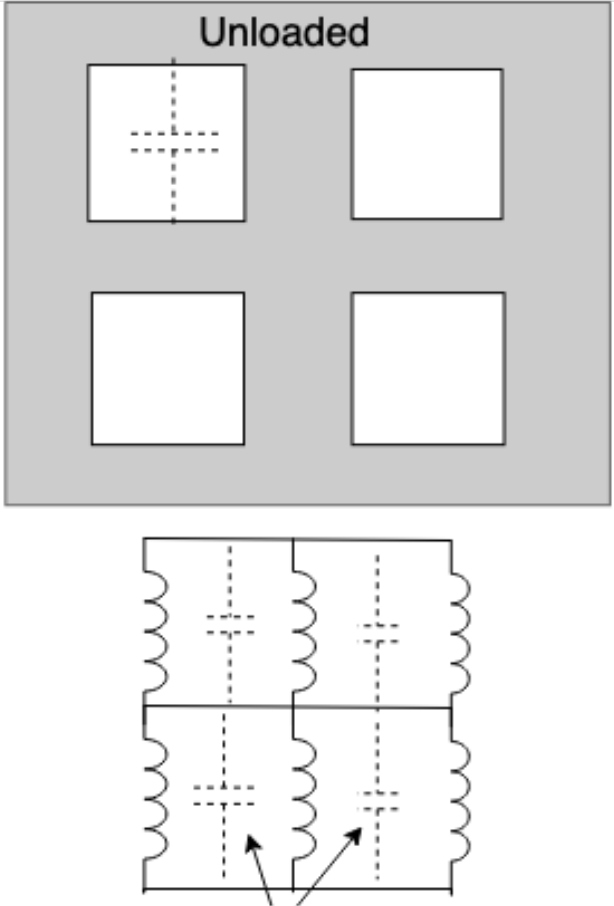

Small Capacitance

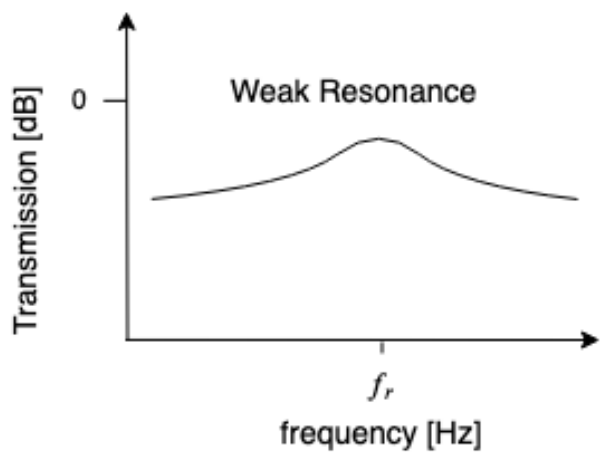

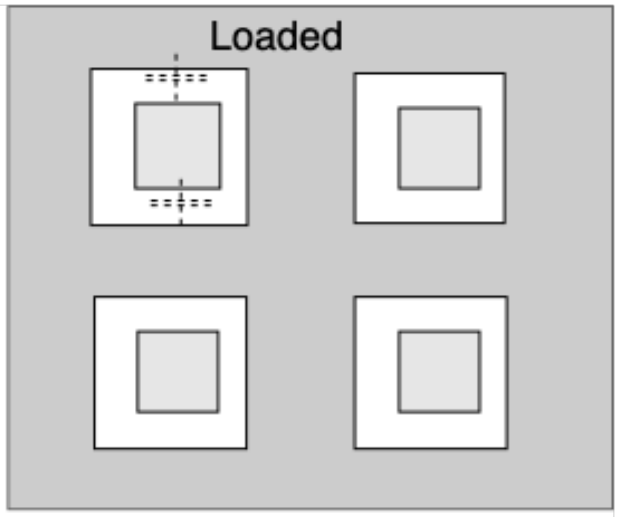

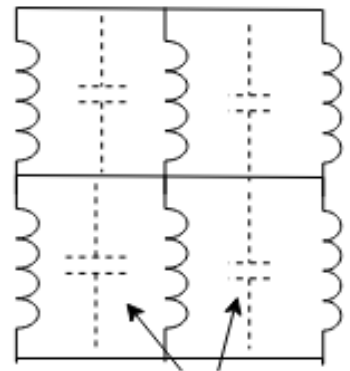

Large Capacitance

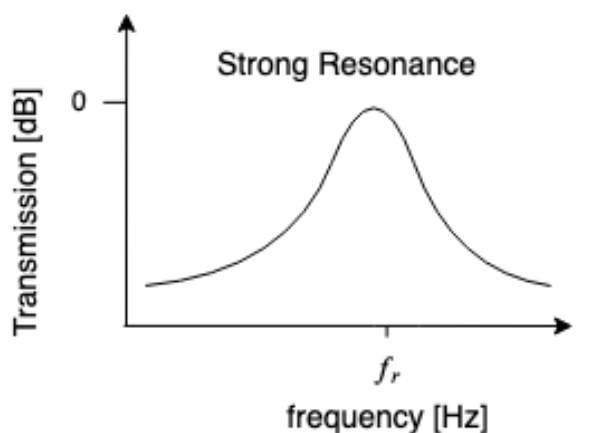

frequency $[\mathrm{Hz}]$

Figure 3.4: Unloaded and Loaded square aperture FSS, their equivalent circuits, and their respective transmission curves. Unloaded FSS shows lesser magnitude resonance compared to loaded FSS [8]. 
Chapter 4

DESIGN

\subsection{Loaded Cross Aperture Configuration}

The loaded cross aperture configuration is chosen as the unit cell in this work. The configuration is adapted from [11] and modified to follow fabrication constraints. This configuration is a cross dipole unit cell placed within a cross shaped aperture. The cross aperture and cross dipole configurations are examined in section 4.3.1. Figure 4.1 below shows cross dipole, cross aperture, and loaded cross aperture FSS unit cell configurations.
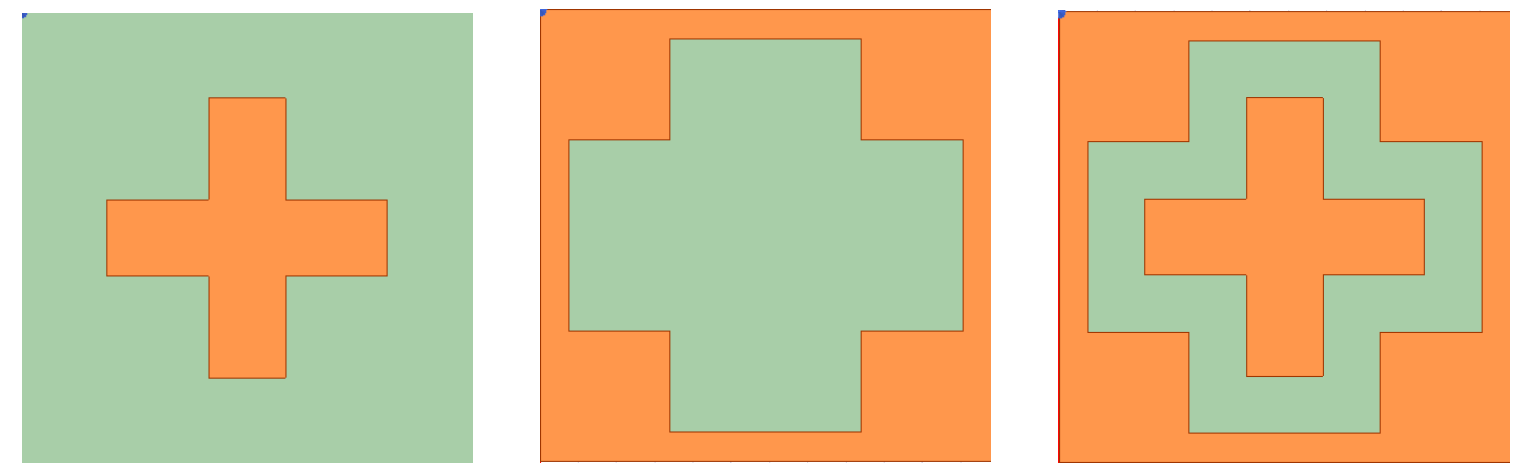

Figure 4.1: Cross dipole (a), cross aperture (b), and loaded cross aperture (c) FSS unit cell configurations. Conducting copper surface (orange), dielectric substrate (green).

The loaded cross aperture configuration provides resonance frequency stability vs. EM wave incident angle. This configuration also enhances design frequency resonance. The stronger resonance enables minimum unit cell dimensions. Reduced unit cell size allows smaller interelement distances, $D_{x}$ and $D_{y}$, which increases FSS resonance frequency stability relative to incident angle. 
The configuration in Figure 4.1c is designed at $f_{r}=79 \mathrm{GHz}$ and $f_{r}=8 \mathrm{GHz}$. A $79 \mathrm{GHz}$ resonance frequency is chosen to place the peak transmission resonance in the IWR1642 chirp signal's center $(77 \mathrm{GHz}-81 \mathrm{GHz})$. The FSS passes frequencies in the chirp signal bandwidth. However, Vector Network Analyzers to record $S_{11}$ reflection and $S_{21}$ transmission measurements are not available. Therefore, an $8 \mathrm{GHz}$ FSS is designed and fabricated to verify operation. Unit cell dimensions (loaded cross aperture) are modified to resonate at $8 \mathrm{GHz}$.

\subsection{HFSS Simulations}

The FSS designs are simulated in HFSS (High Frequency Structure Simulator). The unit cell is defined in figure 4.1c. HFSS simulates FSS using Floquet port excitations. Floquet ports extend a single FSS unit cell to an infinitely repeating structure in the unit cell plane. Floquet ports simulate mode sets that represent plane waves whose propagation direction is set by the frequency, phasing, and periodic structure geometry [1]. These ports simulate transverse electric (TE) and transverse magnetic (TM) modes which define reflection and transmission characteristics for different EM wave polarizations. Master and Slave boundaries define the unit cell boundaries. The Master boundaries are assigned a Slave boundary on the unit cell's opposite side. Each Master/Slave boundary pair forces identical boundary conditions [1]. This is necessary to simulate the unit cell as an infinitely repeating structure. Figure 4.2 below shows Floquet port and Master/Slave pair assignments.

The dielectric substrate used is $127 \mu m$ ( 5 mil) thick FR4 with $\epsilon_{r}=4.4$, and dielectric loss tangent, $\tan \delta=0.02$. The copper thickness is $35 \mu m(1 \mathrm{oz}$. copper $)$. FR4 is selected to minimize cost compared to high frequency dielectric materials, such as Duroid. The manufacturer, Bay Area Circuit's minimum substrate thickness is 5 

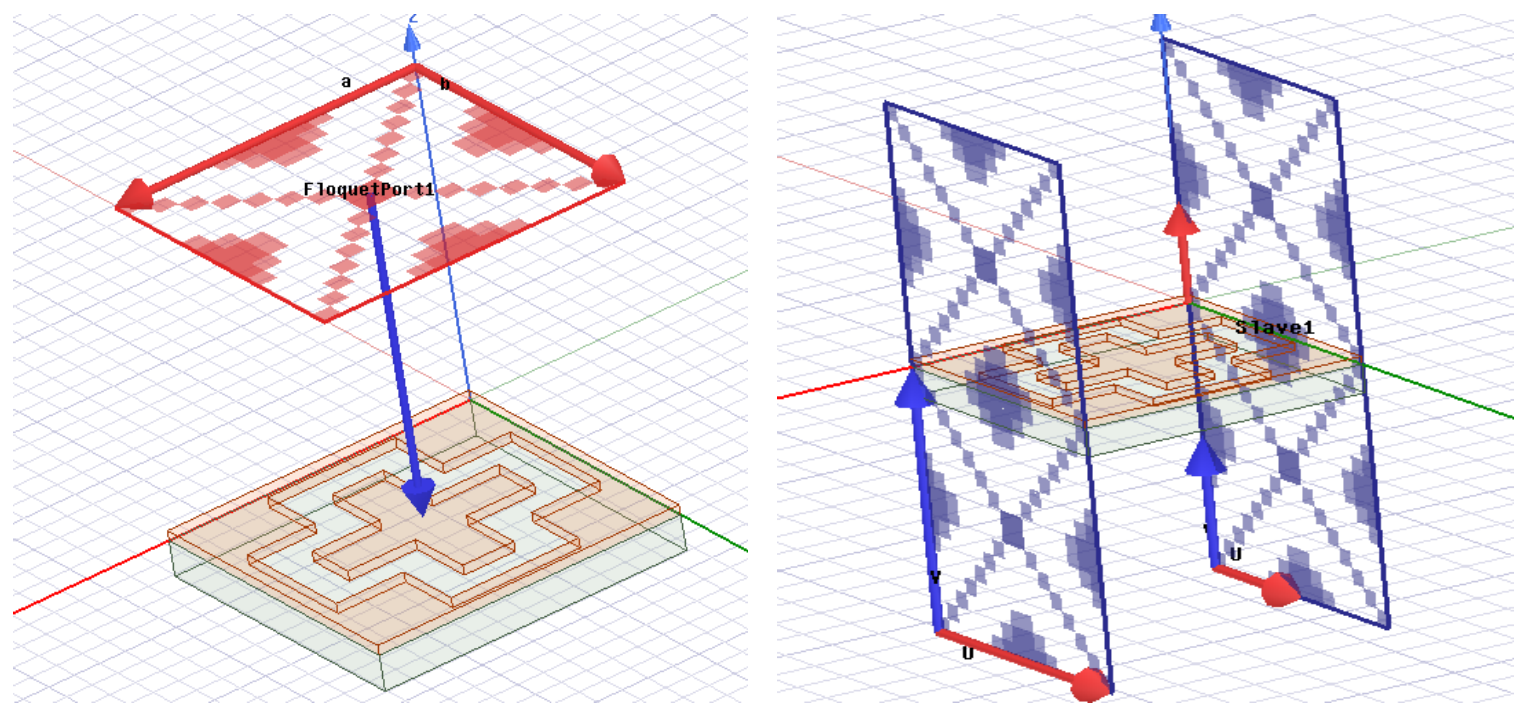

Figure 4.2: HFSS Model, FSS Floquet port simulation (a) and Master/Slave boundary (b).

mils. Section 4.3.2 discusses the dielectric thickness and material simulated effects on the resonant frequency.

\section{3 $\quad 79 \mathrm{GHz}$ design}

Figure 4.3 below shows the $79 \mathrm{GHz}$ FSS unit cell dimensions. The first resonance frequency occurs when the interior element circumference is approximately $\lambda_{r}$.

Figure 4.4 below shows the $79 \mathrm{GHz}$ FSS simulated transmission and reflection curves. The peak resonance occurs at $f_{r}=79.5 \mathrm{GHz}$. The transmission's $-1 \mathrm{~dB}$ bandwidth is approximately $17.4 \mathrm{GHz}$.

Figure 4.5 below shows the $79 \mathrm{GHz}$ FSS simulated $S_{11}$ reflection response at various incident plane wave angles. This simulation indicates that an increase in incident angle has minimal effect on the FSS's resonant frequency. However, the resonance bandwidth decreases as incident angle increases. 


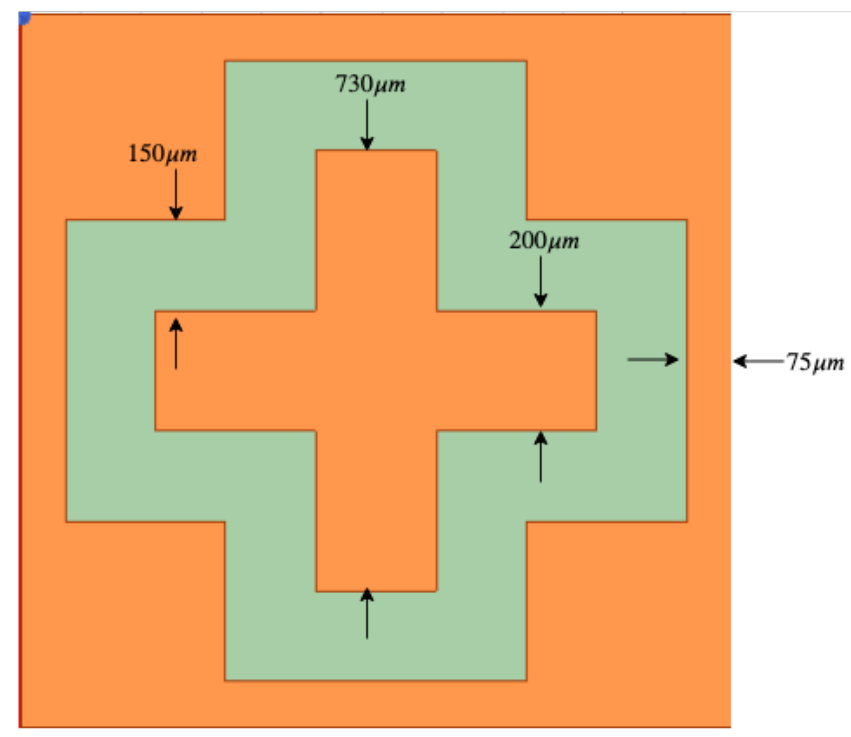

Figure 4.3: $79 \mathrm{GHz}$ FSS unit cell dimensions, $127 \mu m$ (5 mil) thick FR4 (green), 1 oz. copper(orange).

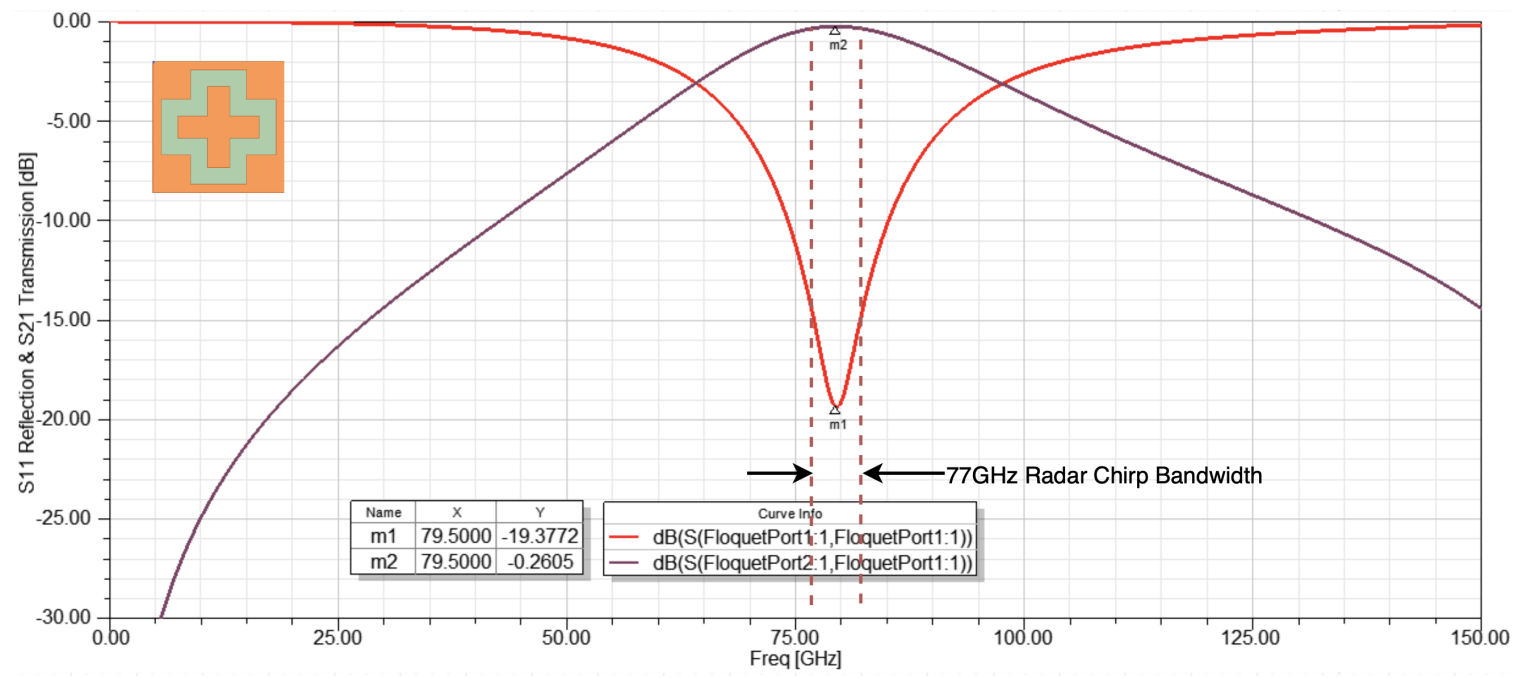

Figure 4.4: $79 \mathrm{GHz}$ FSS Reflection (red) and Transmission (purple) HFSS simulations, incident plane wave propagation direction perpendicular to FSS, $127 \mu m$ (5 mil) thick FR4.

\subsubsection{Cross Dipole and Cross Aperture}

Figures 4.6 and 4.7 show $79 \mathrm{GHz}$ FSS simulations with the cross dipole and cross aperture configurations, respectively. The unit cell dimensions in Figure 4.6 are the 


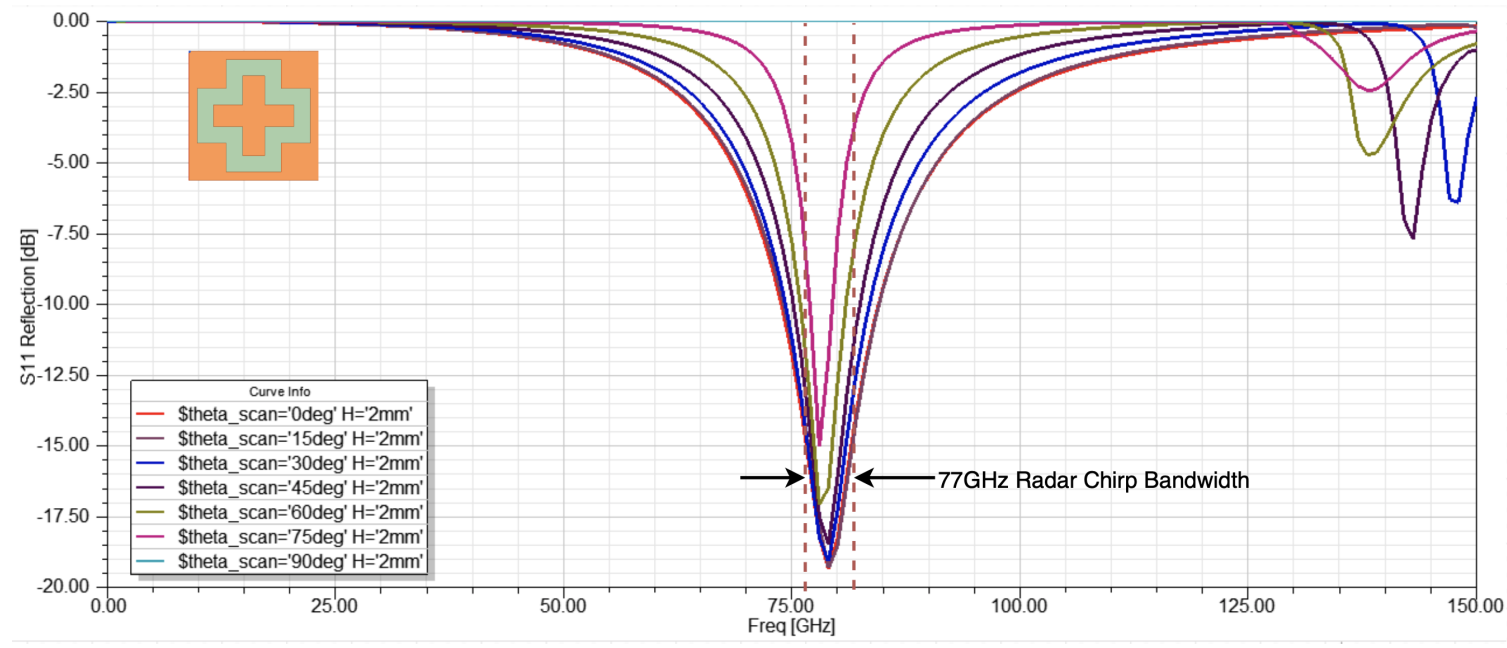

Figure 4.5: $79 \mathrm{GHz}$ FSS simulated resonance versus frequency at various incident angles, $127 \mu \mathrm{m}$ (5 mil) thick FR4.

same as the inner cross dipole in Figure 4.3. The unit cell dimensions in Figure 4.7 are the same as the outer aperture dimensions in Figure 4.3.

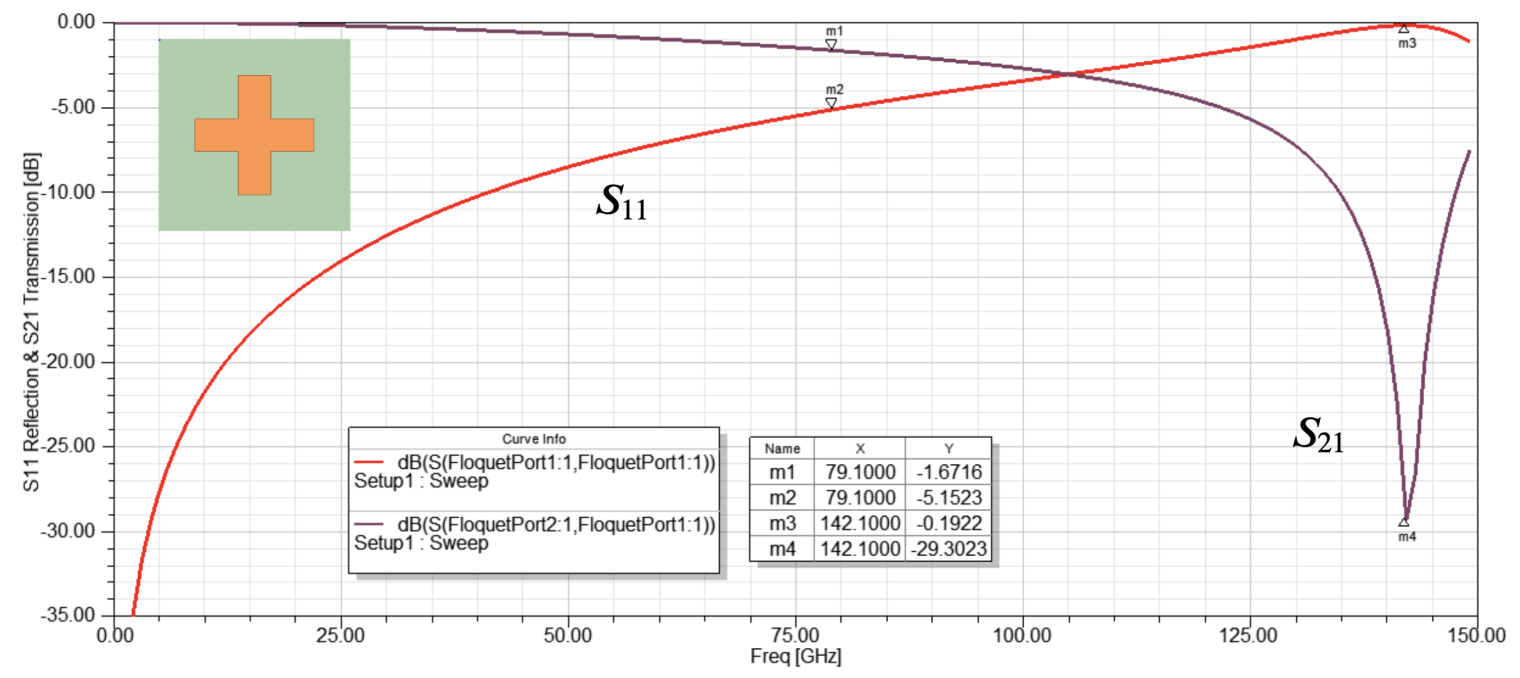

Figure 4.6: $79 \mathrm{GHz}$ FSS cross dipole HFSS simulation, Figure 4.1a configuration, $S_{21}$ (purple), $S_{11}$ (red), $127 \mu m$ (5 mil) thick FR4.

Figure 4.6 confirms that patch like FSS elements perform as bandstop filters. The $S_{21}$ transmission curve null and $S_{11}$ reflection peak at $142.1 \mathrm{GHz}$ indicates band stop filter performance. 


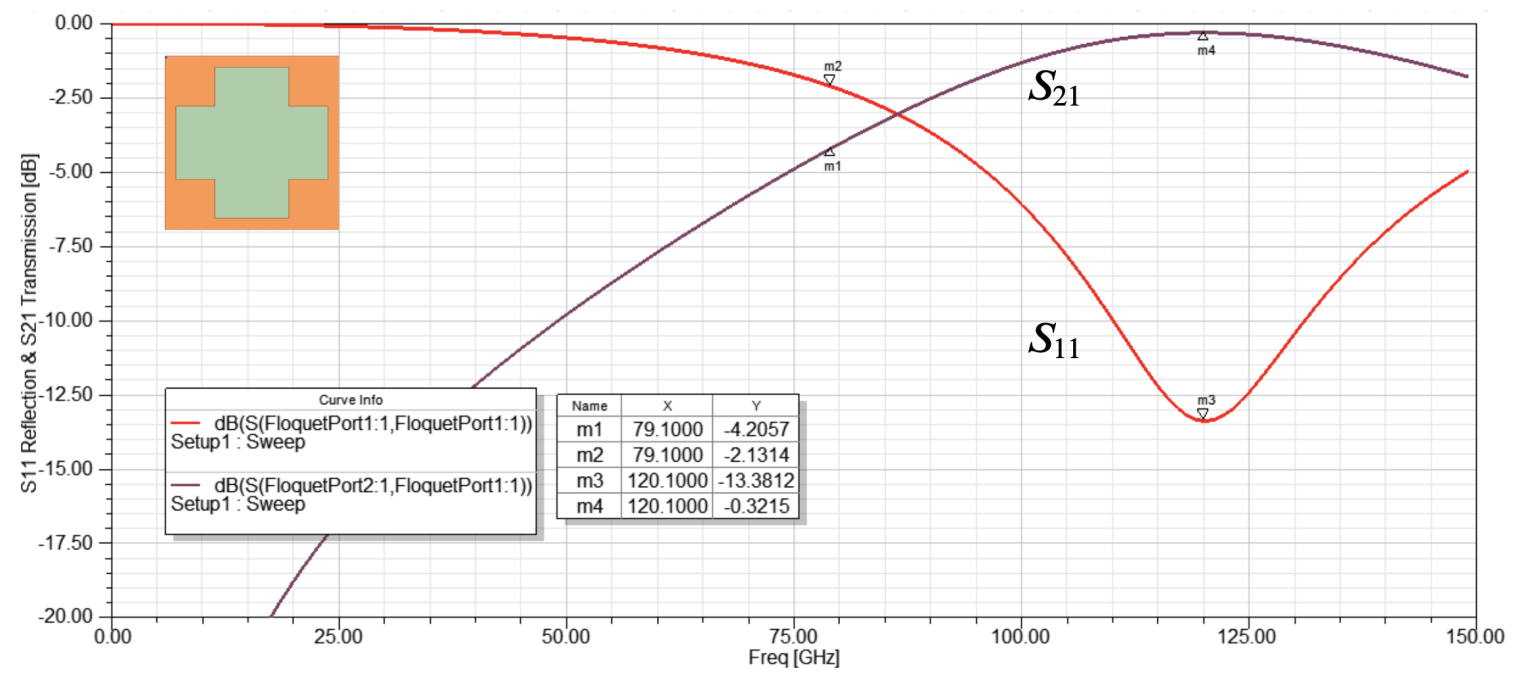

Figure 4.7: $79 \mathrm{GHz}$ FSS cross aperture HFSS simulation, Figure 4.1b configuration, $S_{21}$ (purple), $S_{11}$ (red), $127 \mu m$ (5 mil) thick FR4.

Figure 4.7 shows resonance at $f=120.1 \mathrm{GHz}$. The cross aperture simulation shows a much greater bandwidth than the loaded cross aperture simulation in figure 4.4. This indicates loading the aperture unit cell with the cross dipole creates a stronger resonance.

\subsubsection{Dielectric Substrate Effects}

Figure 4.8 below shows the loaded cross aperture configuration with no dielectric material. This simulation demonstrates the FSS's resonant frequency with no dielectric. Peak resonance at $f_{r}=122.5 G H z$ confirms resonant frequency shift by one-sided dielectric discussed in section 3.4. Figure 4.9 shows the loaded cross aperture $\left|S_{11}\right|$ reflection curves vs. dielectric thickness, H_diel. The simulation indicates that resonance magnitude decreases and bandwidth increases as dielectric material thickness increases.

Figure 4.10 shows the $79 \mathrm{GHz}$ FSS loaded cross aperture design simulation with Duroid substrate material, dielectric constant $\epsilon_{r}=2.2$. Therefore, resonance frequency increases in agreement with Section 3.4. 


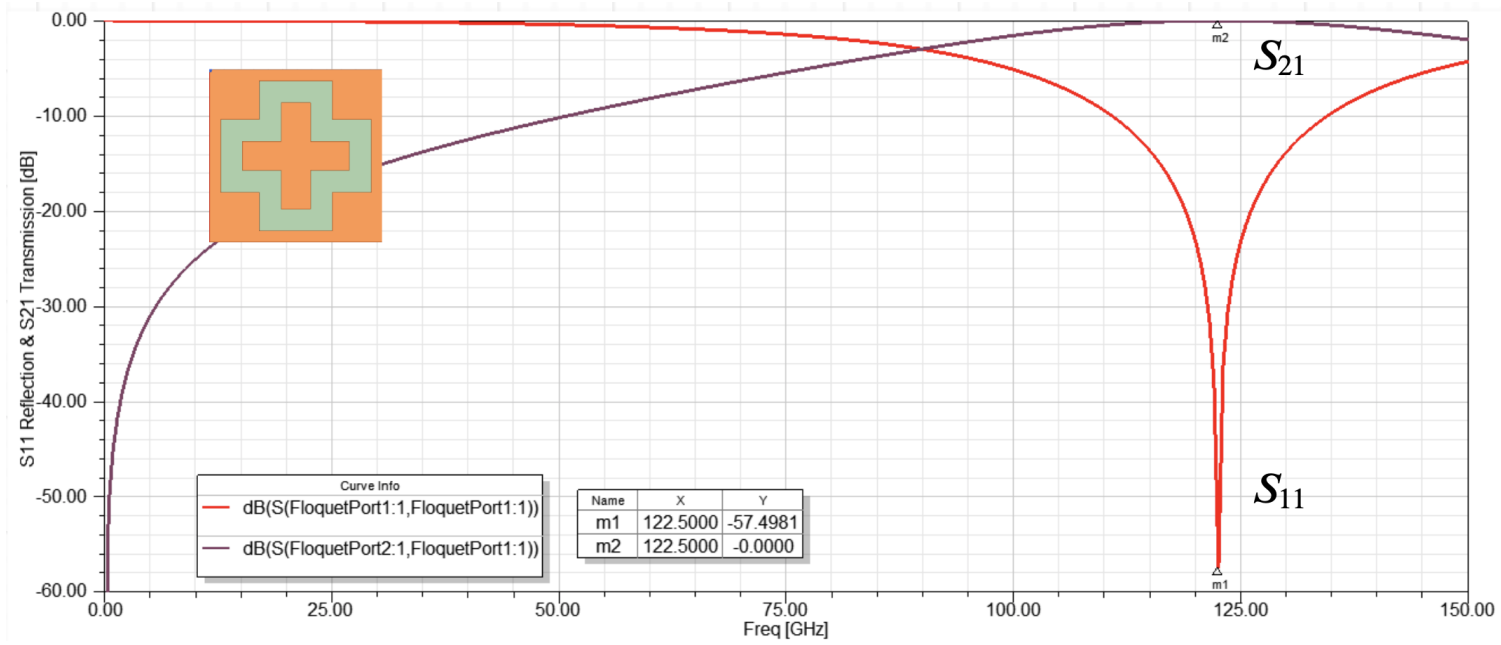

Figure 4.8: $79 \mathrm{GHz}$ FSS loaded cross aperture configuration, dielectric substrate removed, $S_{21}$ (purple), $S_{11}$ (red).

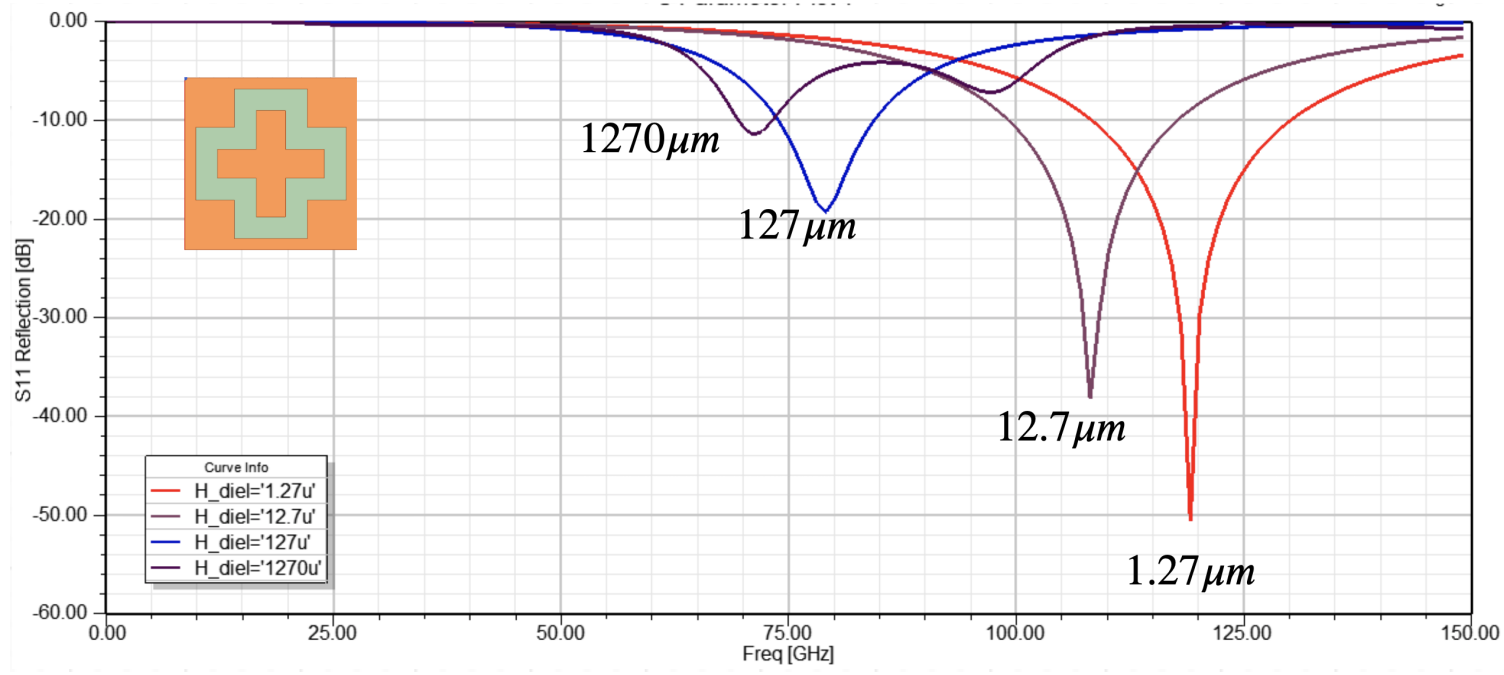

Figure 4.9: $79 \mathrm{GHz}$ FSS loaded cross aperture configuration $S_{11}$ reflection curves, dielectric substrate thickness, H_diel, swept logarithmically $1.27 \mu \mathrm{m}$ - $1270 \mu m$.

\subsubsection{Inter-element Spacing Analysis}

Figure 4.11 below shows $79 \mathrm{GHz}$ loaded cross aperture configuration $S_{11}$ reflection curves vs. inter-element spacing G. The simulation indicates that FSS resonance magnitude and bandwidth decrease with increased spacing. This simulation is consistent with section element spacing effects discussed in section 3.3. 


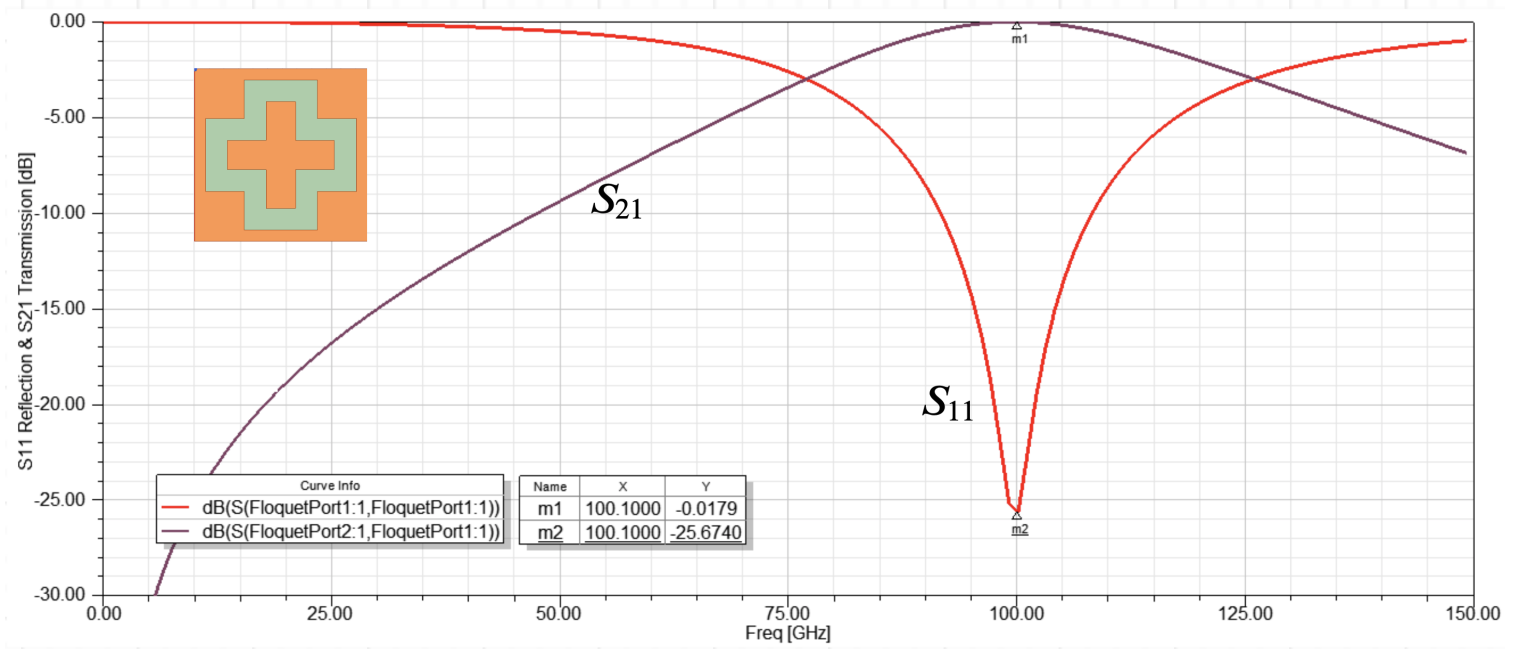

Figure 4.10: $79 \mathrm{GHz}$ FSS loaded cross aperture configuration, Duroid substrate $\left(\epsilon_{r}=2.2\right), S_{21}$ (purple), $S_{11}$ (red).

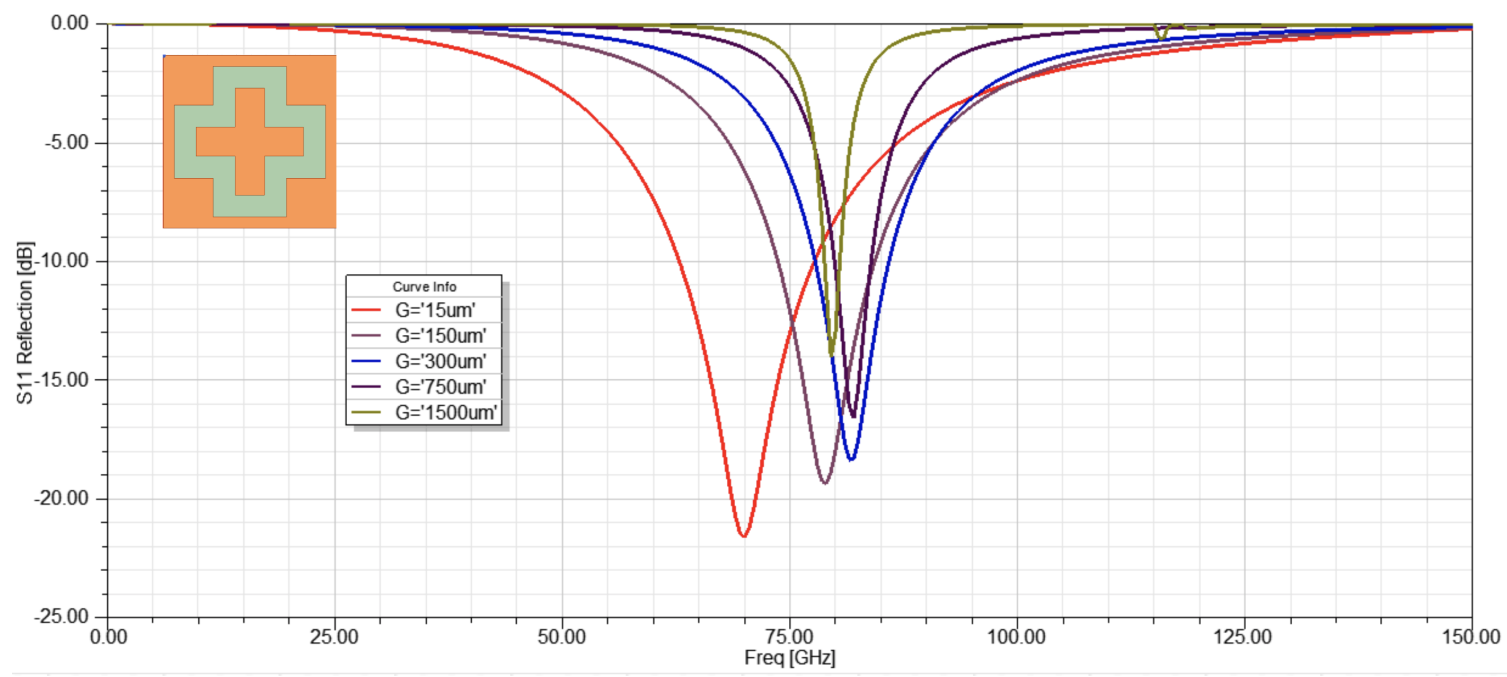

Figure 4.11: $79 \mathrm{GHz}$ FSS loaded cross aperture configuration $S_{11}$ reflection curves, Inter-element spacing, G, swept $15 \mu \mathrm{m}-1500 \mu \mathrm{m}$.

\subsubsection{Tolerance Analysis}

$79 \mathrm{GHz}$ FSS unit cell dimension variations determine performance sensitivity. Figures 4.13 and 4.14 below show simulations of the $79 \mathrm{GHz}$ FSS with each feature size increased and decreased 10\%, respectively. The inner dipole and aperture width, length, and gap size are adjusted. The simulations show resonant frequency shift $\Delta f_{r}=-7 G H z$ and $\Delta f_{r}=+8.5 G H z$, respectively. These simulations demonstrate 
that fabrication accuracy at the micron scale are necessary to correctly resonate FSS in the mmWave wavelength range. Figure 4.12 shows the dimensions used in the simulation shown in figure 4.13

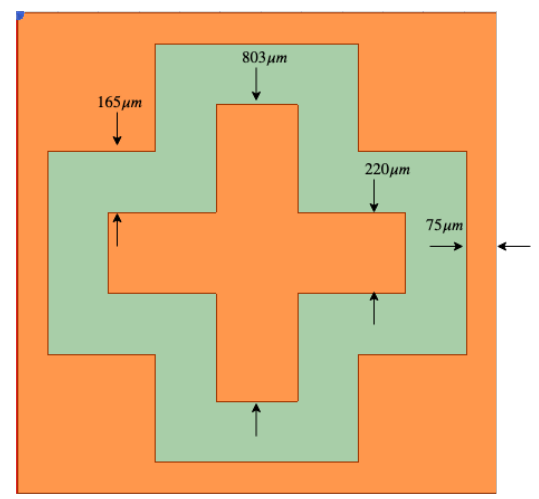

Figure 4.12: $79 \mathrm{GHz}$ FSS loaded cross aperture configuration dimensions, inner dipole and aperture width, length, and gap size increased by $10 \%$.

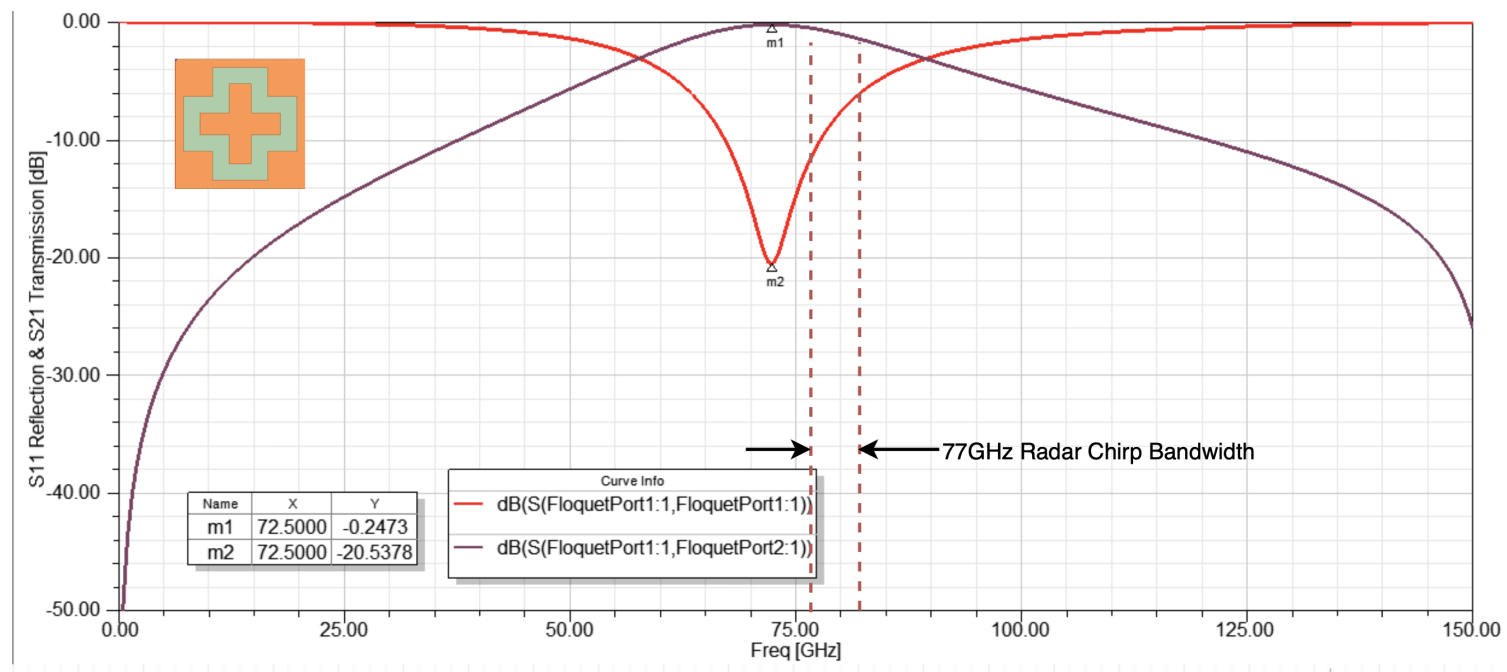

Figure 4.13: $79 \mathrm{GHz}$ FSS loaded cross aperture configuration HFSS simulation, inner dipole and aperture width, length, and gap size increased by $10 \%, S_{21}$ (purple), $S_{11}$ (red).

\section{4 $8 \mathrm{GHz}$ design}

Figure 4.15 shows the $8 \mathrm{GHz}$ FSS unit cell dimensions. This design also utilizes the loaded cross aperture configuration. 


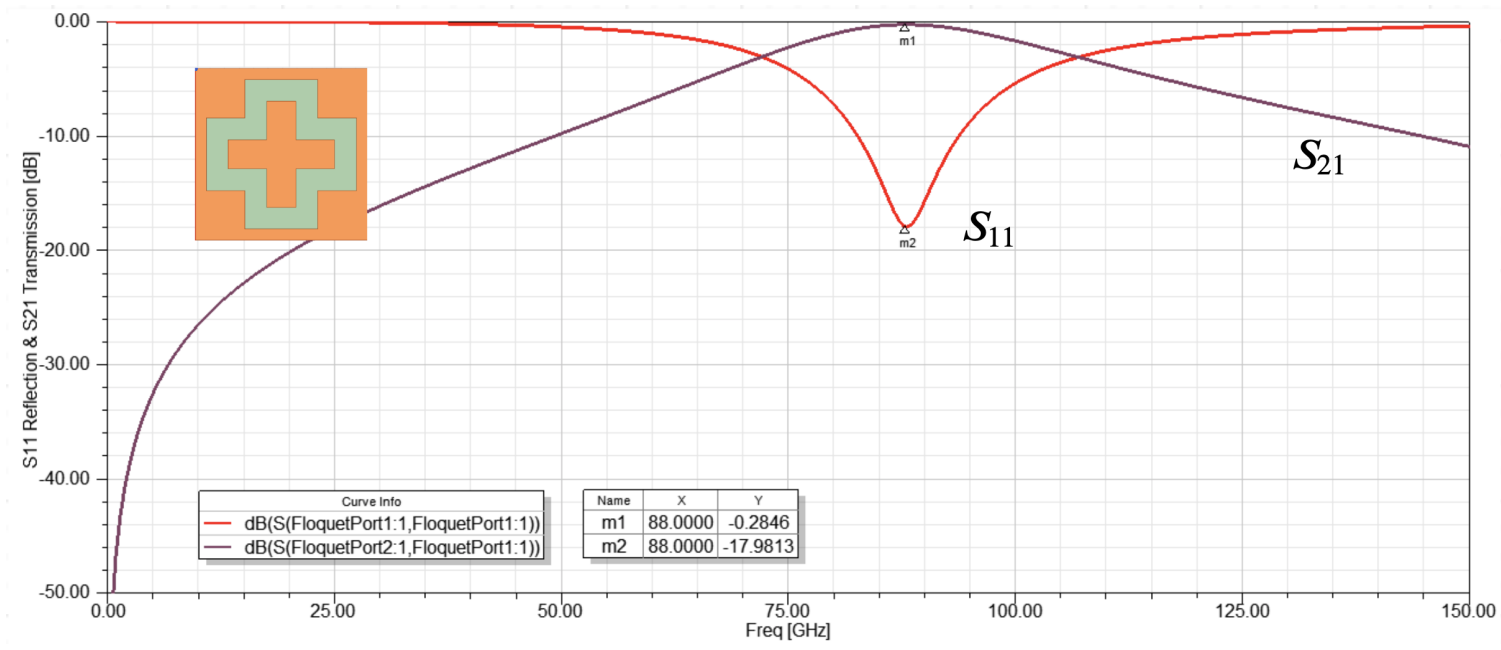

Figure 4.14: $79 \mathrm{GHz}$ FSS loaded cross aperture configuration, inner dipole and aperture width, length, and gap size decreased by $10 \%, S_{21}$ (purple), $S_{11}$ (red).

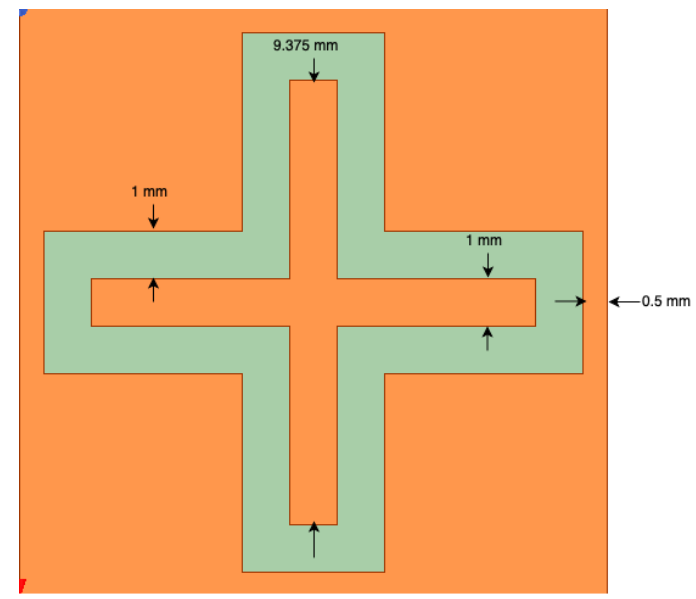

Figure 4.15: $8 \mathrm{GHz}$ FSS unit cell dimensions.

Figure 4.16 shows the $8 \mathrm{GHz}$ FSS simulated $S_{11}$ reflection and $S_{21}$ transmission curves. The first and second peak resonances are $f_{r 1}=8.07 \mathrm{GHz}$ and $f_{r 2}=17.55 \mathrm{GHz}$, respectively. The $8 \mathrm{GHz}$ design demonstrates lower reflection magnitude at first resonance than the $79 \mathrm{GHz}$ FSS. The $8 \mathrm{GHz}$ FSS first resonance $S_{11}$ magnitude compared to the $-10 \mathrm{~dB}$ BW is greater than that of the $79 \mathrm{GHz}$ FSS. Table 4.1 below compares the resonance strength of each design. 


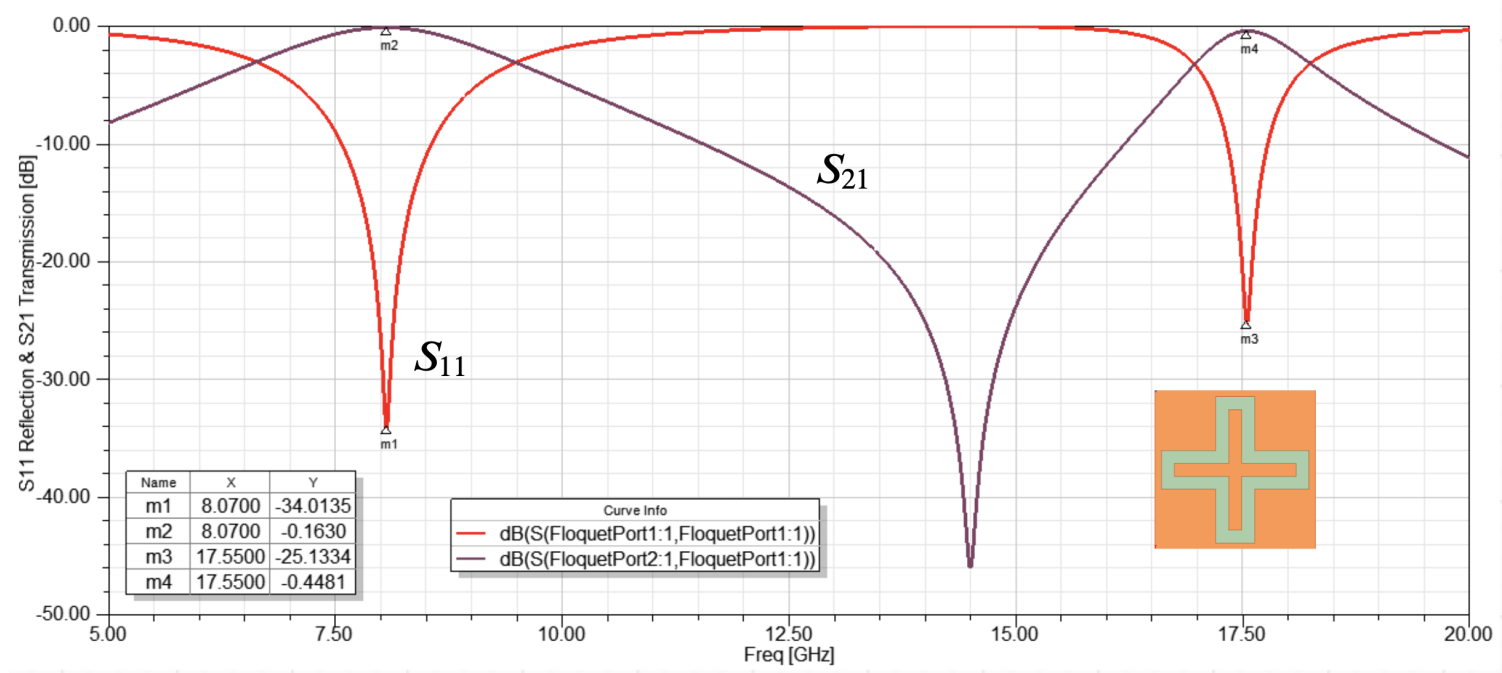

Figure 4.16: $8 \mathrm{GHz}$ FSS HFSS simulated $S_{11}$ reflection (red) and $S_{21}$ transmission (purple).

Table 4.1: Simulated first resonance strength indicator, $8 \mathrm{GHz}$ FSS and $79 \mathrm{GHz}$ FSS

\begin{tabular}{|c|c|c|}
\hline First Resonance Strength Indicator & $8 \mathrm{GHz}$ FSS & $79 \mathrm{GHz}$ FSS \\
\hline$\frac{f_{r}}{B W_{-10 d B, S_{11}}}$ & 8.07 & 7.23 \\
\hline
\end{tabular}

Figure 4.17 below shows the $8 \mathrm{GHz}$ FSS simulated $S_{11}$ reflection curves vs. incident plane wave angles. The variable theta_scan represents EM plane wave's incident angle upon the FSS. The simulation indicates minimal $\left|S_{11}\right|$ minimum (first resonance) deviation vs. incident angle.

\subsection{Fabrication}

The unit cells are expanded to create unit cell arrays. The arrays are exported from HFSS as Gerber files necessary to manufacturing. Figure 4.18 below shows the $8 \mathrm{GHz}$ and $79 \mathrm{GHz}$ array Gerber files used in manufacturing. The $8 \mathrm{GHz}$ FSS is an 8 x 8 unit cell array, while the $79 \mathrm{GHz}$ FSS is a 40 x 40 unit cell array. Figure 4.19 below displays the fabricated $79 \mathrm{GHz}$ and $8 \mathrm{GHz}$ boards. The unit cells are repeated in a 


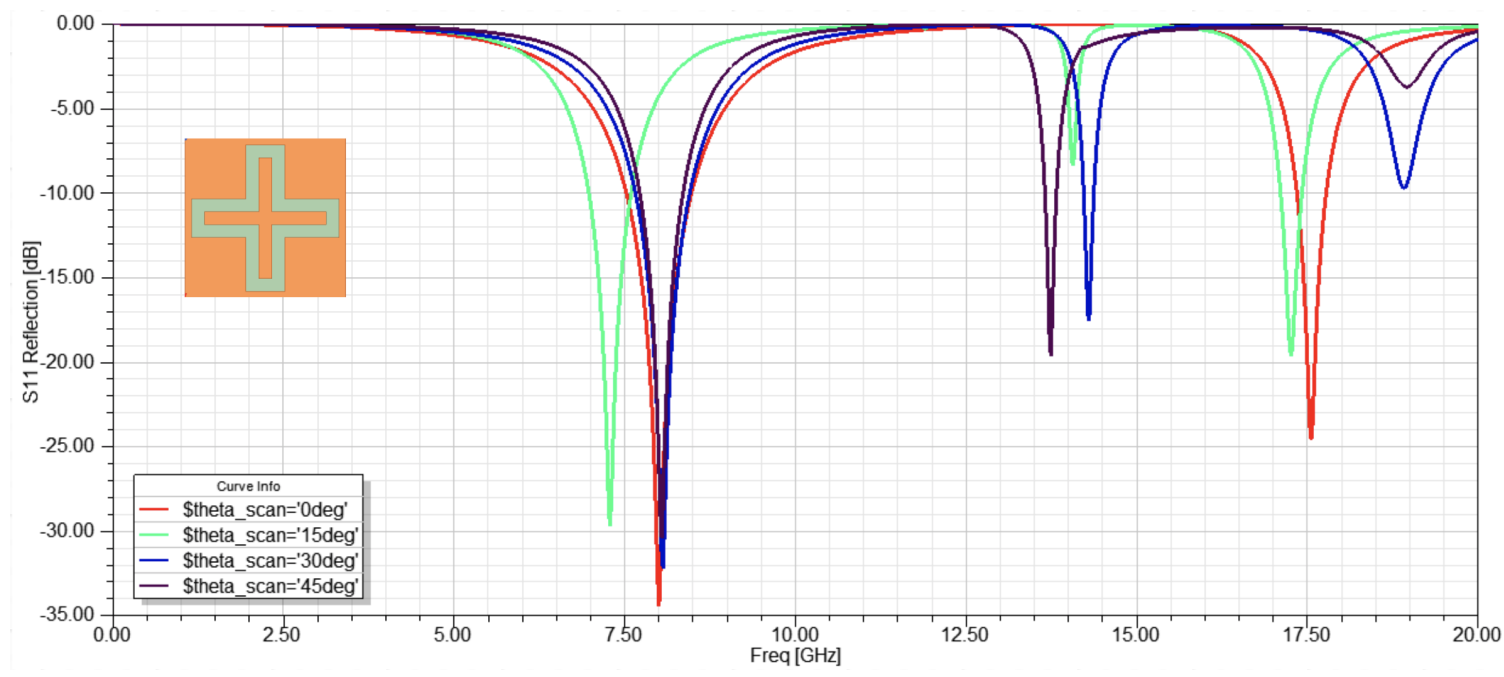

Figure 4.17: $8 \mathrm{GHz}$ FSS simulated $S_{11}$ reflection curves, incident angle sweep, \$theta_scan, $0^{\circ}-45^{\circ}$, $15^{\circ}$ steps.

2D plane to create the FSS. One sided FR4 dielectric with thickness $127 \mu m$, and $1 \mathrm{oz}$ copper are used in this design. Bay Area Circuits, PCB fabricator, created the FSSs. 


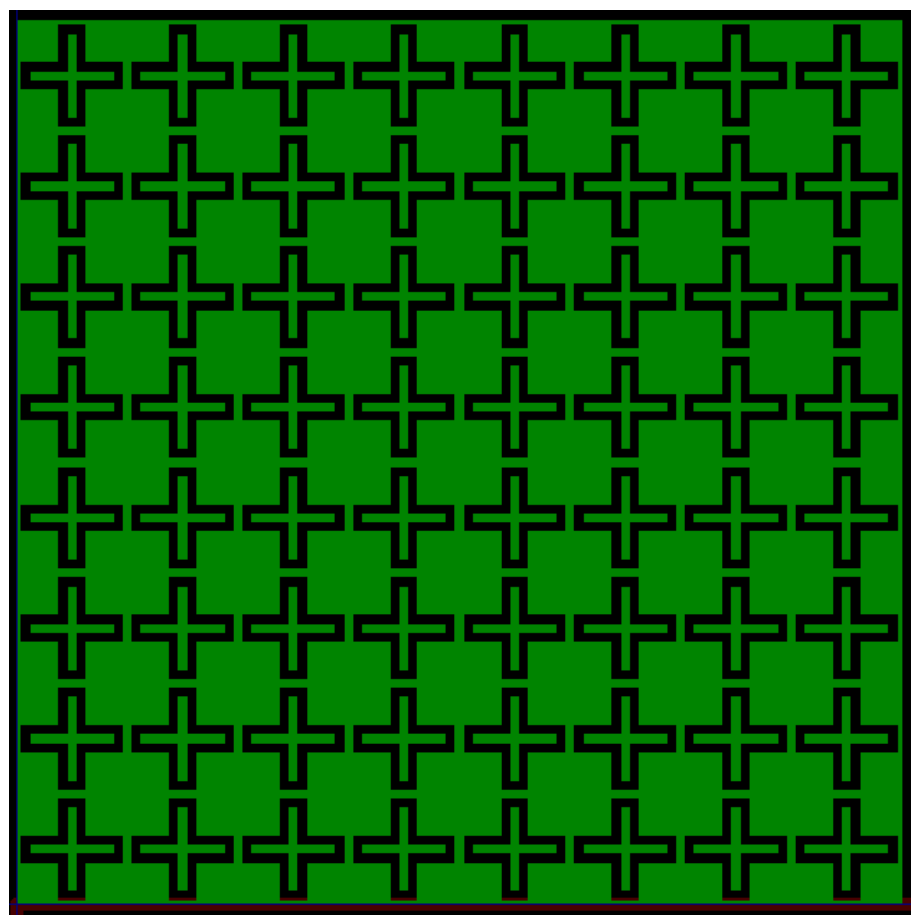

(a) $8 \mathrm{GHz}$ FSS Gerber file layout

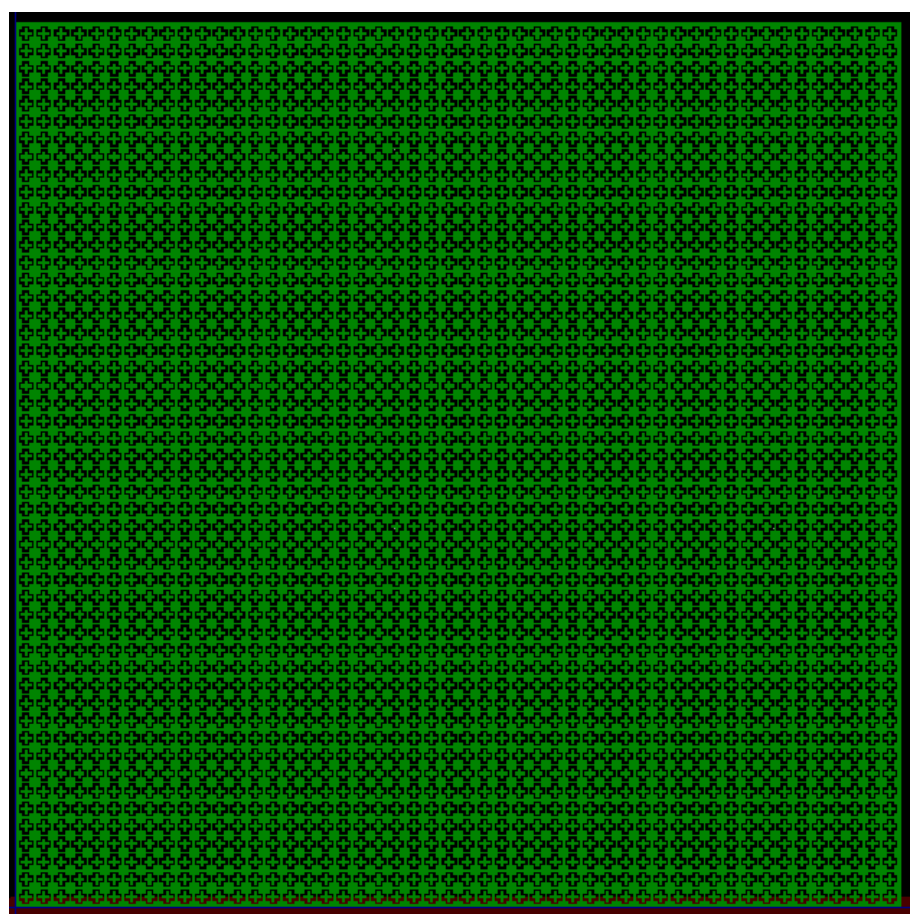

(b) $79 \mathrm{GHz}$ FSS Gerber file layout

Figure 4.18: Gerber File layouts 


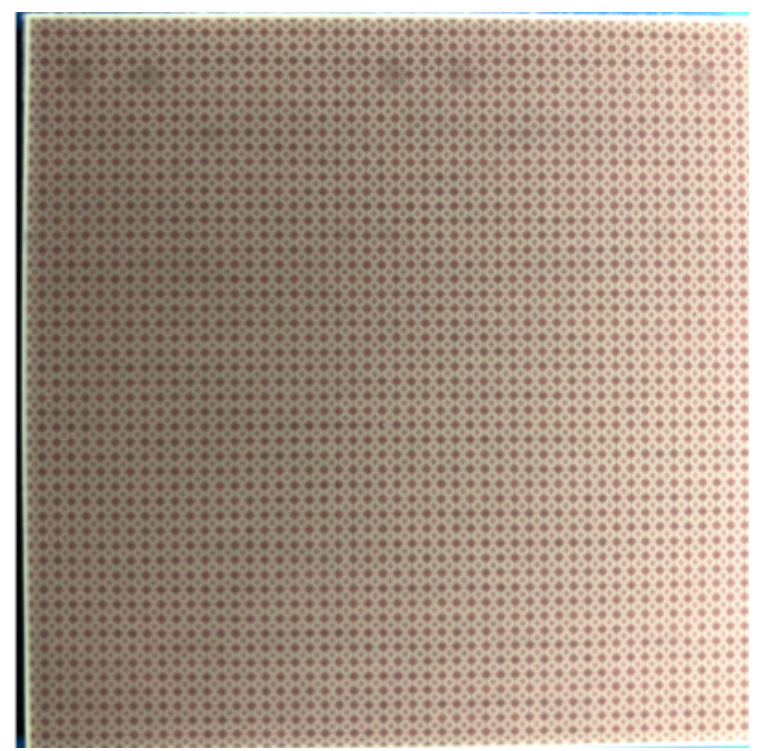

(a) $79 \mathrm{GHz}$ FSS, Total Cross Section = $6 \mathrm{~cm} \times 6 \mathrm{~cm}$

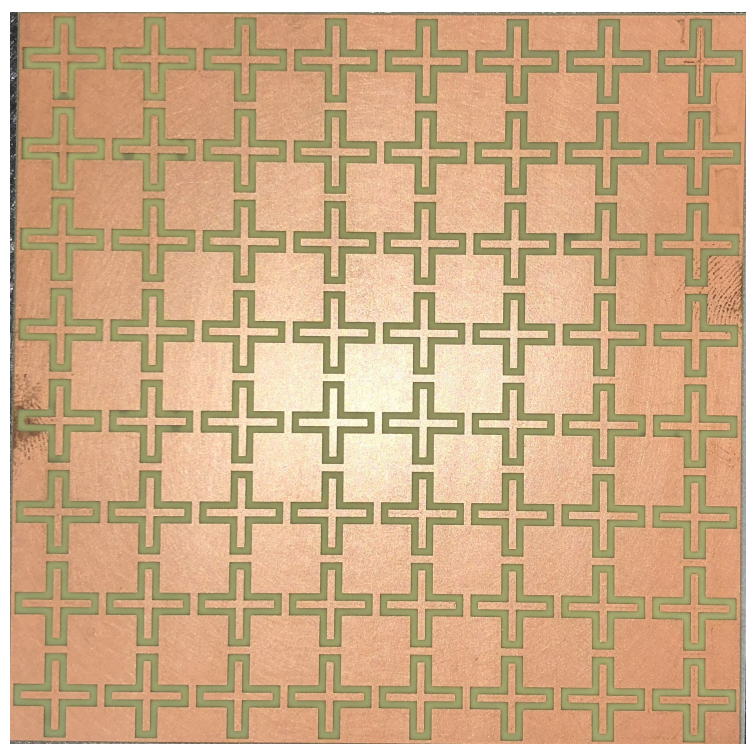

(b) $8 \mathrm{GHz}$ FSS, Total Cross Section = $10 \mathrm{~cm} \times 10 \mathrm{~cm}$

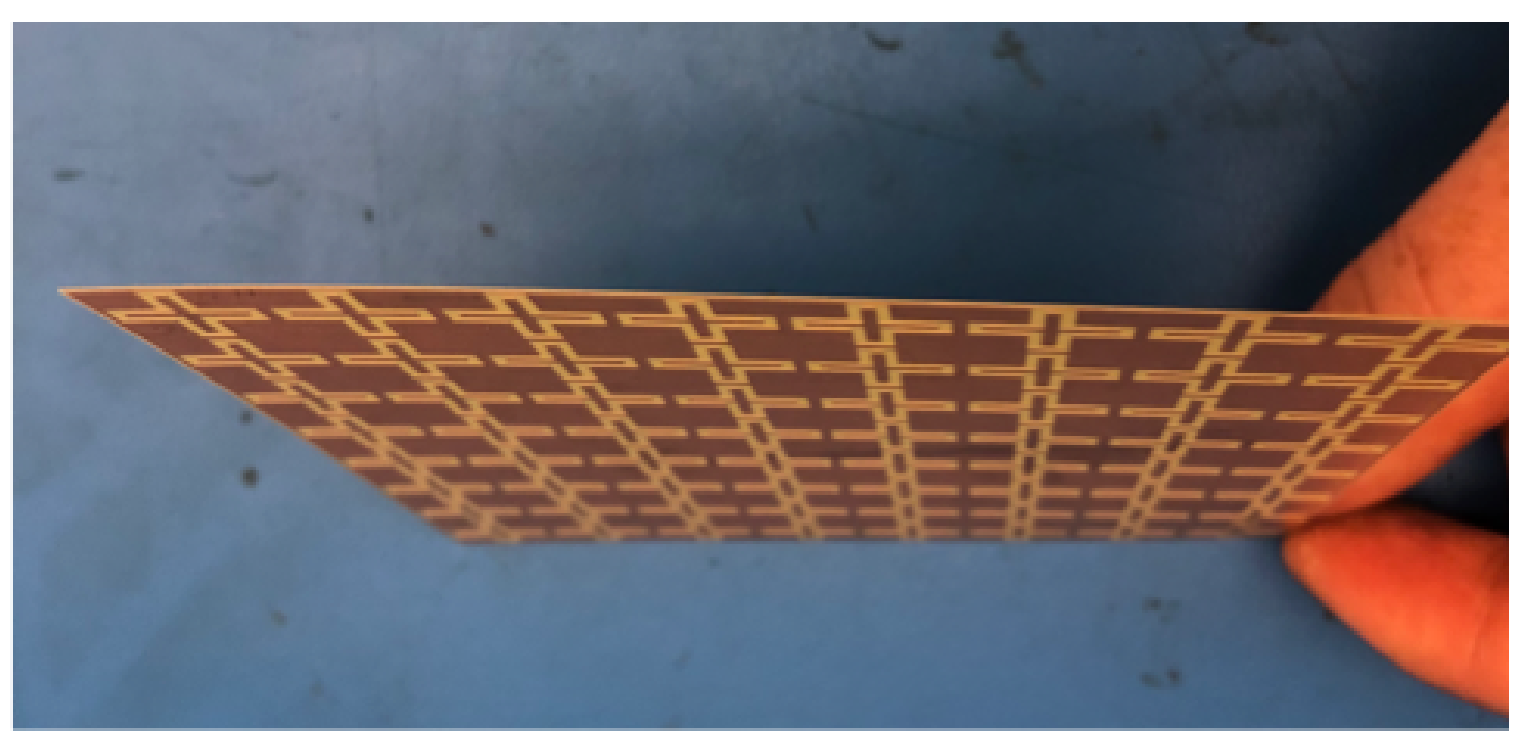

(c) $8 \mathrm{GHz}$ FSS, Side Angle View

Figure 4.19: Fabricated FSS designs. 
Chapter 5

RESULTS

\section{$5.18 \mathrm{GHz}$ FSS}

\subsection{1 $8 \mathrm{GHz}$ FSS Testing Methodology}

The $8 \mathrm{GHz}$ FSS is tested in the anechoic chamber. The FSS is placed directly between two Narda model $6417.05 \mathrm{GHz}$ - $10 \mathrm{GHz}$ standard gain horn antennas. The reflection and transmission characteristics are measured using an Anritsu MS4624B Vector Network Analyzer (VNA). Figures 5.1 and 5.2 show the $8 \mathrm{GHz}$ FSS experimental setup.

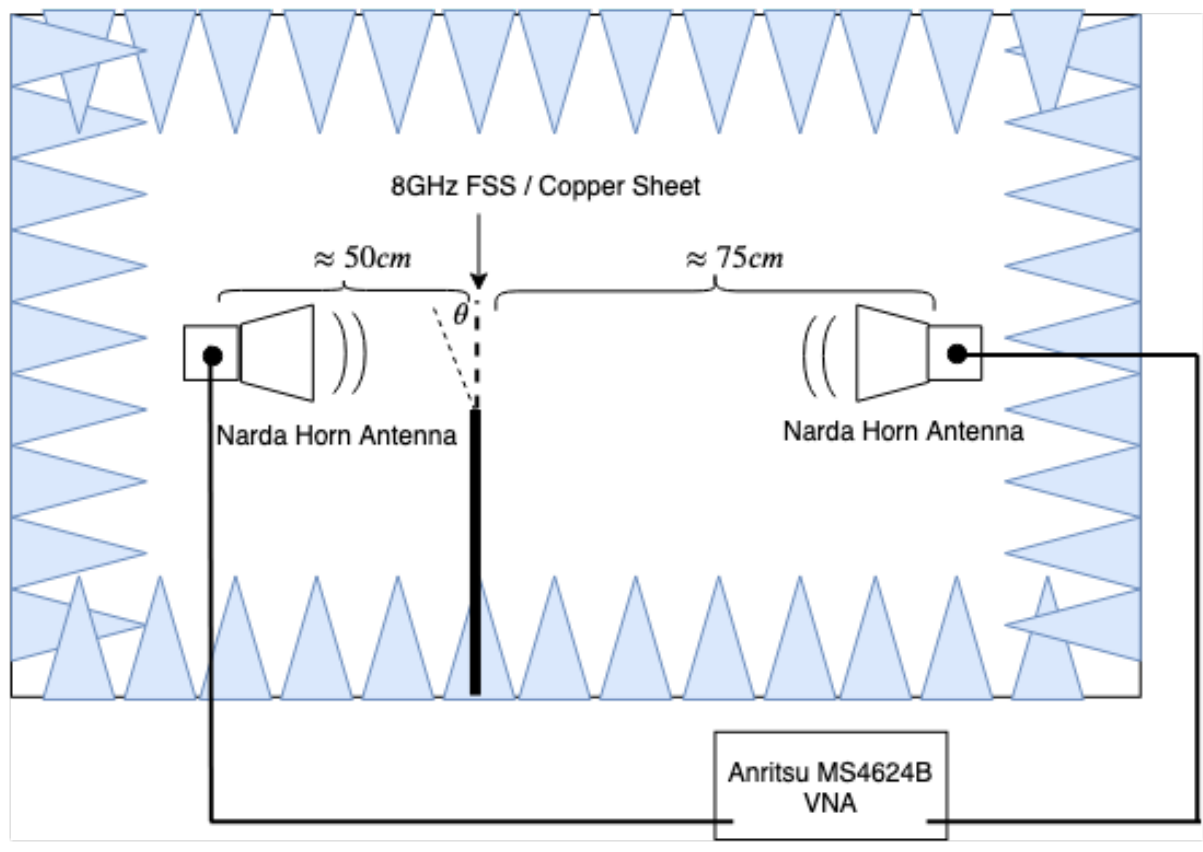

Figure 5.1: $8 \mathrm{GHz}$ FSS experimental setup diagram, Chamber approximately $2 \mathrm{~m}$ x $3 \mathrm{~m}$ (not to scale). 


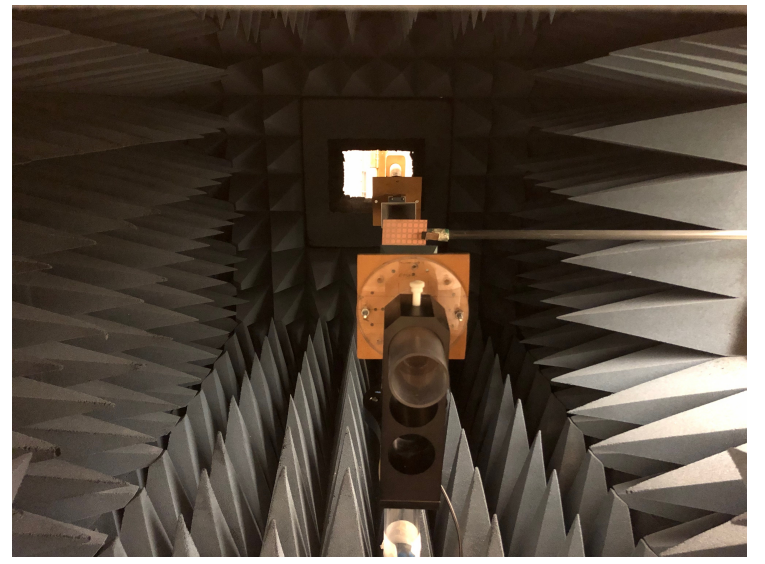

(a)

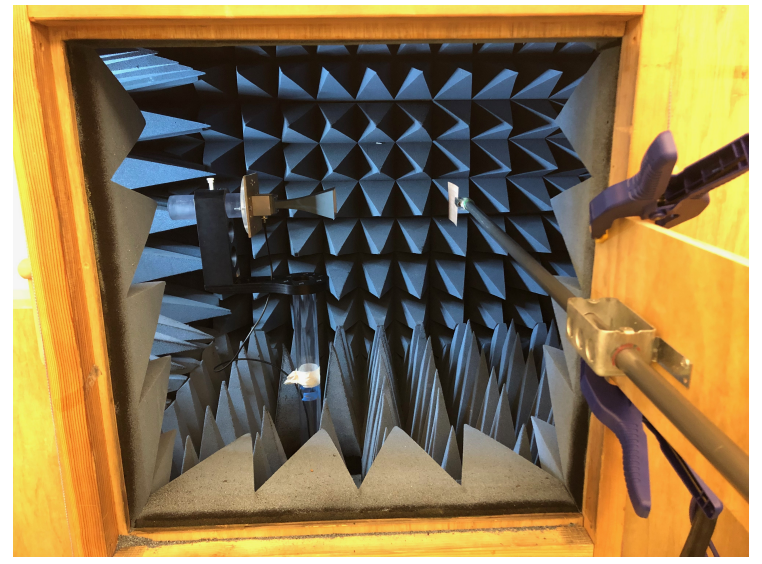

(b)

Figure 5.2: $8 \mathrm{GHz}$ FSS experimental setup, straight on view (a), side view (b).

\subsubsection{Reflection Characteristics}

Reflection measurements were recorded using the $8 \mathrm{GHz}$ FSS and a square $10 \mathrm{~cm}$ x $10 \mathrm{~cm}$ copper sheet cut to the $8 \mathrm{GHz}$ FSS dimensions. Figure 5.3 below shows the copper sheet and $8 \mathrm{GHz}$ FSS measured $S_{11}$ reflection. The reflection measurement comparison shows minimal difference in the response. Therefore, the FSS resonant frequency cannot be determined with this measurement.

To isolate $\Gamma_{2}$ shown in Figure 5.4, time domain measurement techniques are utilized. Time domain reflectometry (TDR) filtering is utilized to more accurately determine the FSS resonant frequency. This technique applies a bandpass filter to the measured $S_{11}$ time domain response. This attenuates the measured response outside the filter's passband. This filtering is referred to as TDR gating. The passband is chosen corresponding to the FSS and copper sheet distance from the horn antenna's SMA connection. Figure 5.5 below shows the general block diagram for the time domain filtering technique. The VNA executes these measurements and calculations to produced the filtered responses. 


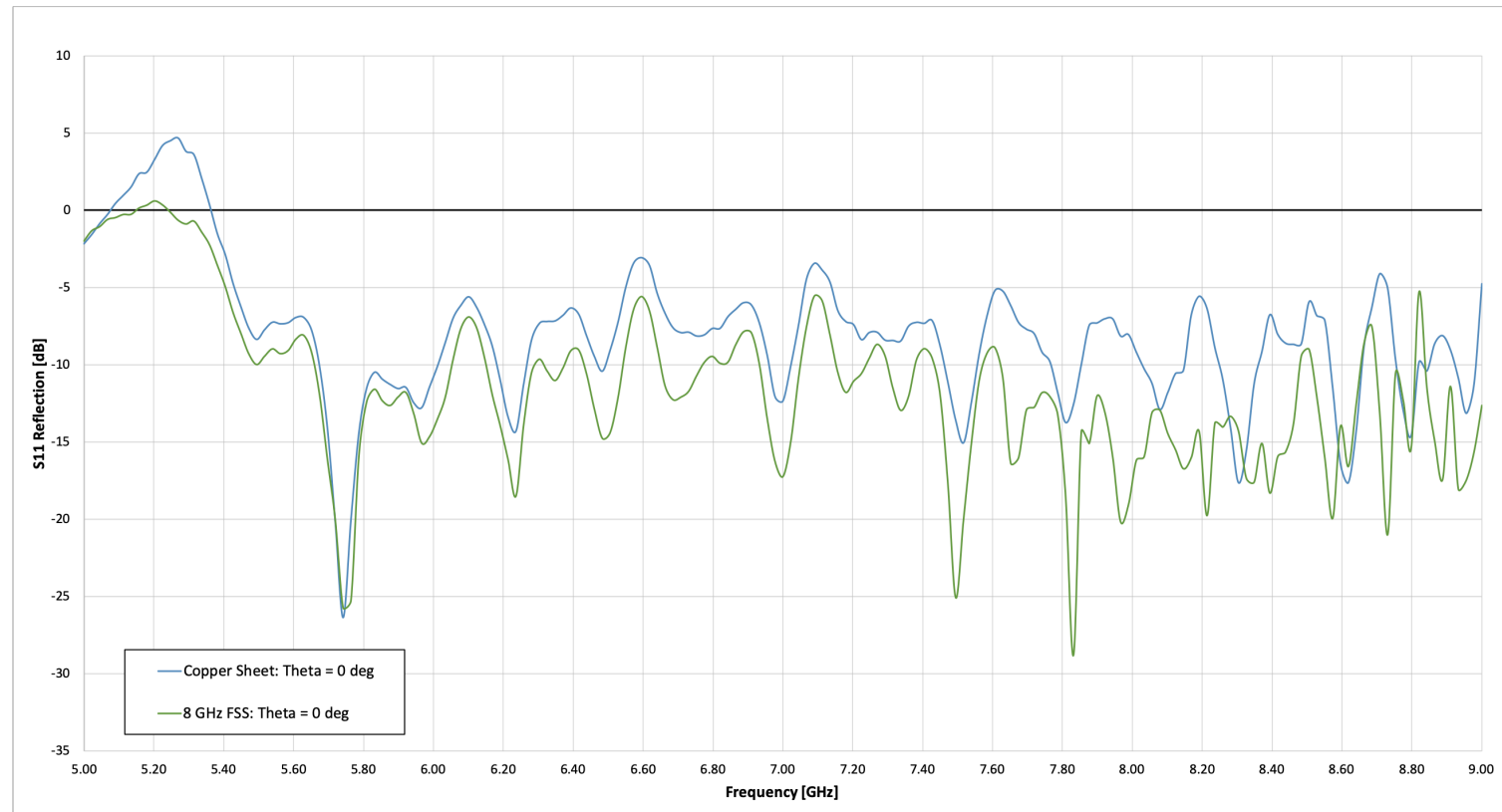

Figure 5.3: Measured $S_{11}$ vs. Frequency, Copper Sheet (blue), 8 GHz FSS (green).

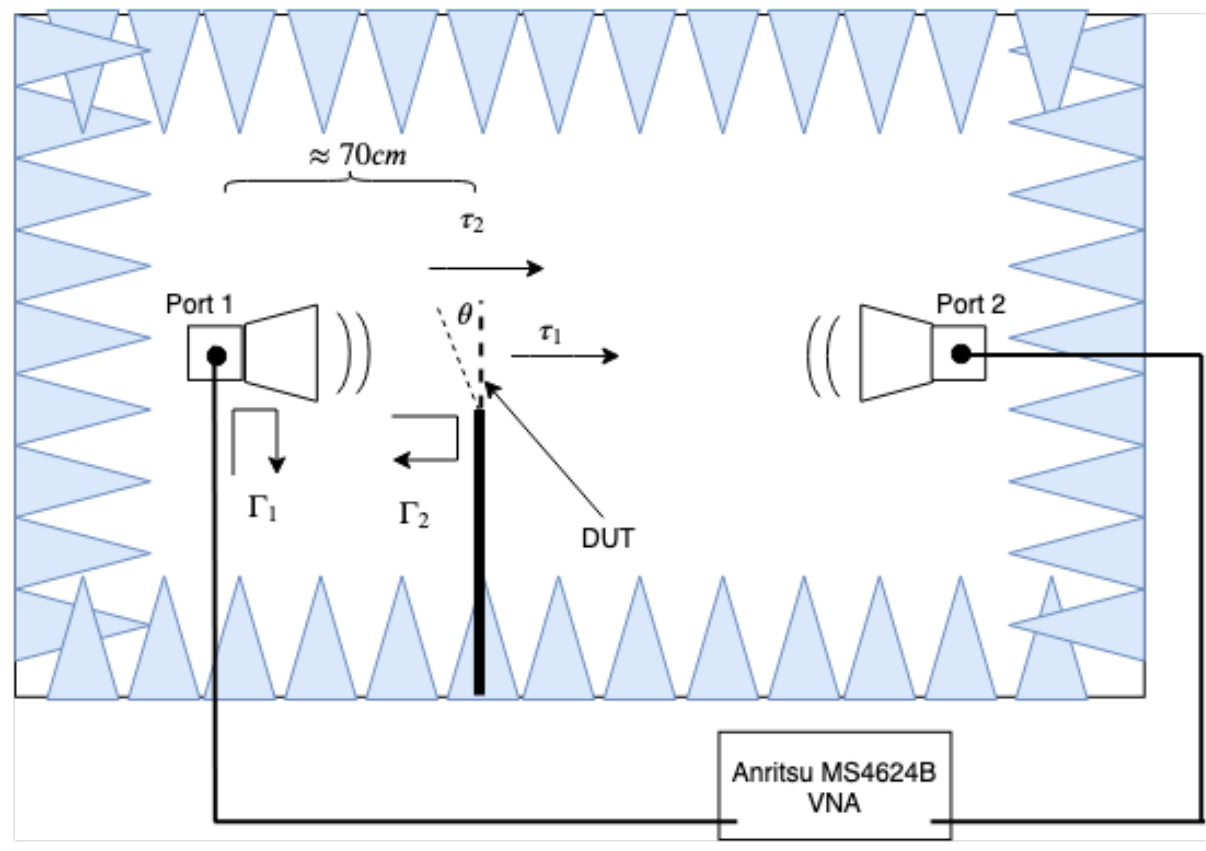

Figure 5.4: $8 \mathrm{GHz}$ FSS experimental Setup Diagram depicting reflection and transmission coefficients.

Figure 5.6 below shows the measured $S_{11}$ response vs. distance from the SMA connection. It shows there are significant reflections that affect the $S_{11}$ response 


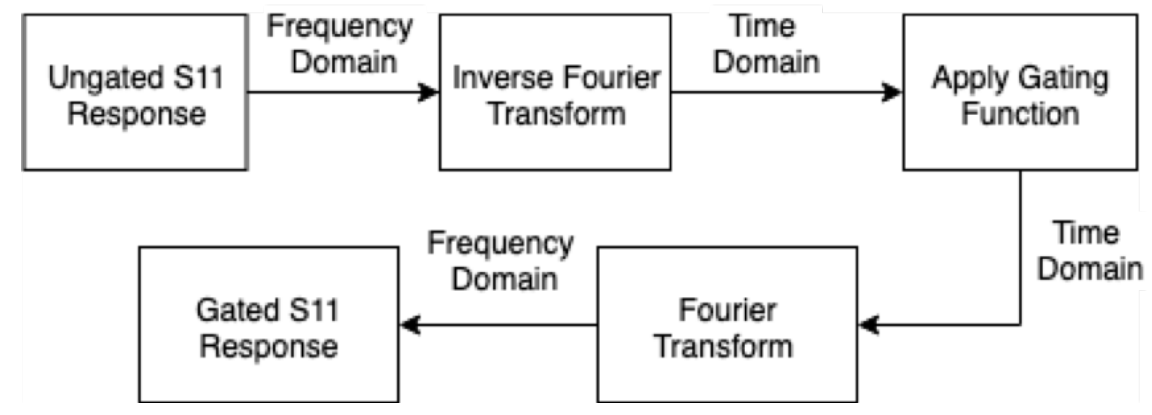

Figure 5.5: High level time domain filtering block diagram.

surrounding $0 \mathrm{~cm}$. This indicates that the SMA junction with the Narda horn antenna is the source of these reflections. Therefore, the TDR gating bandpass filter is applied to the $S_{11}$ response corresponding to the sheet's distance from the SMA connection.

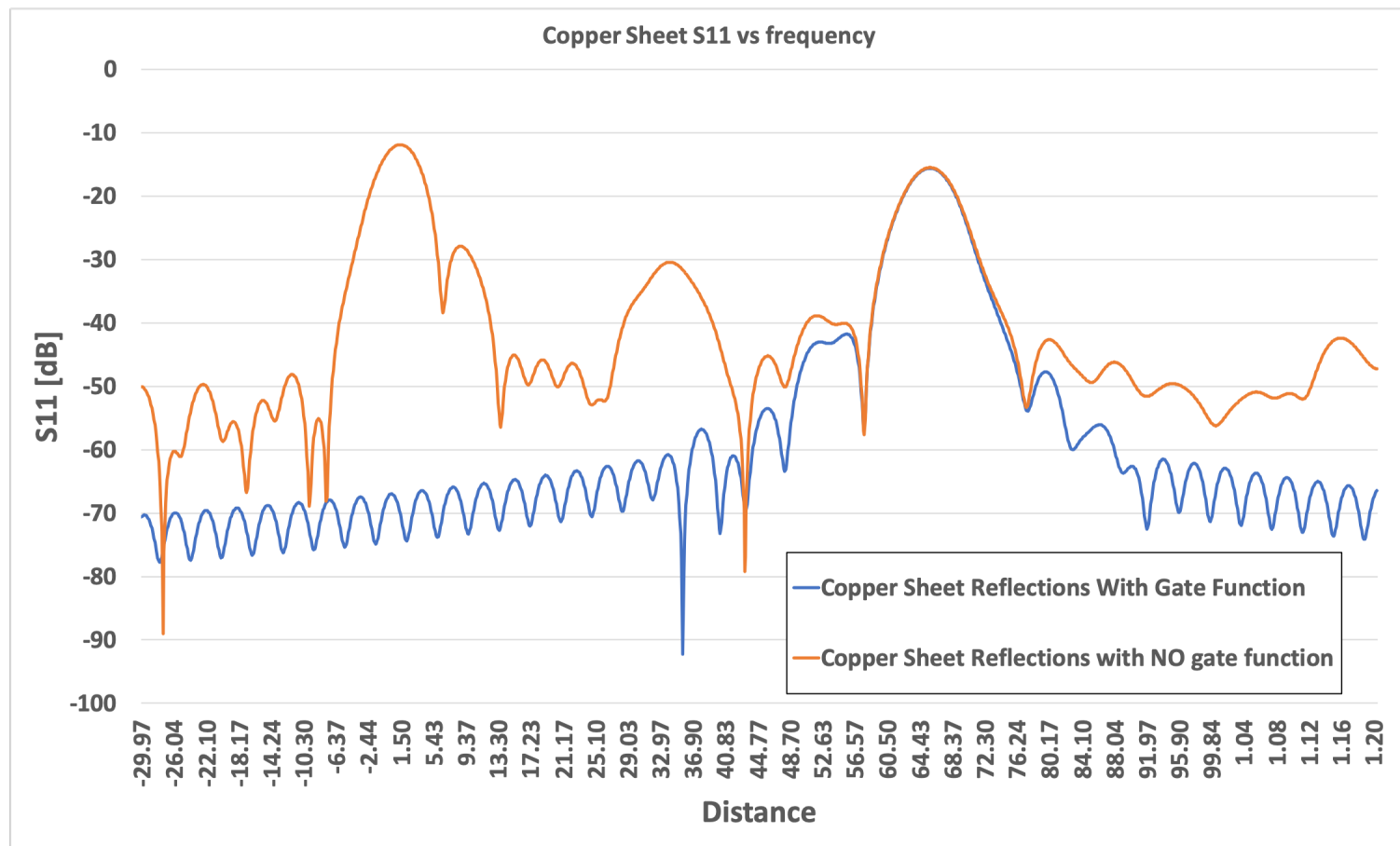

Figure 5.6: Measured $S_{11}$ reflection measurement vs. distance, Copper Sheet with NO TDR gate filter (orange), Copper Sheet with TDR gate function (blue).

Figure 5.7 shows the measured copper sheet and $8 \mathrm{GHz}$ FSS $S_{11}$ response for $f=5-9 \mathrm{GHz}$ using the TDR gating. The $8 \mathrm{GHz}$ FSS response shows $\left|S_{11}\right|=$ 
$-71.936 \mathrm{~dB}$ at $f_{r}=8.12 \mathrm{GHz}$. This is $53.39 \mathrm{~dB}$ less than the copper sheet $S_{11}$ at this frequency.

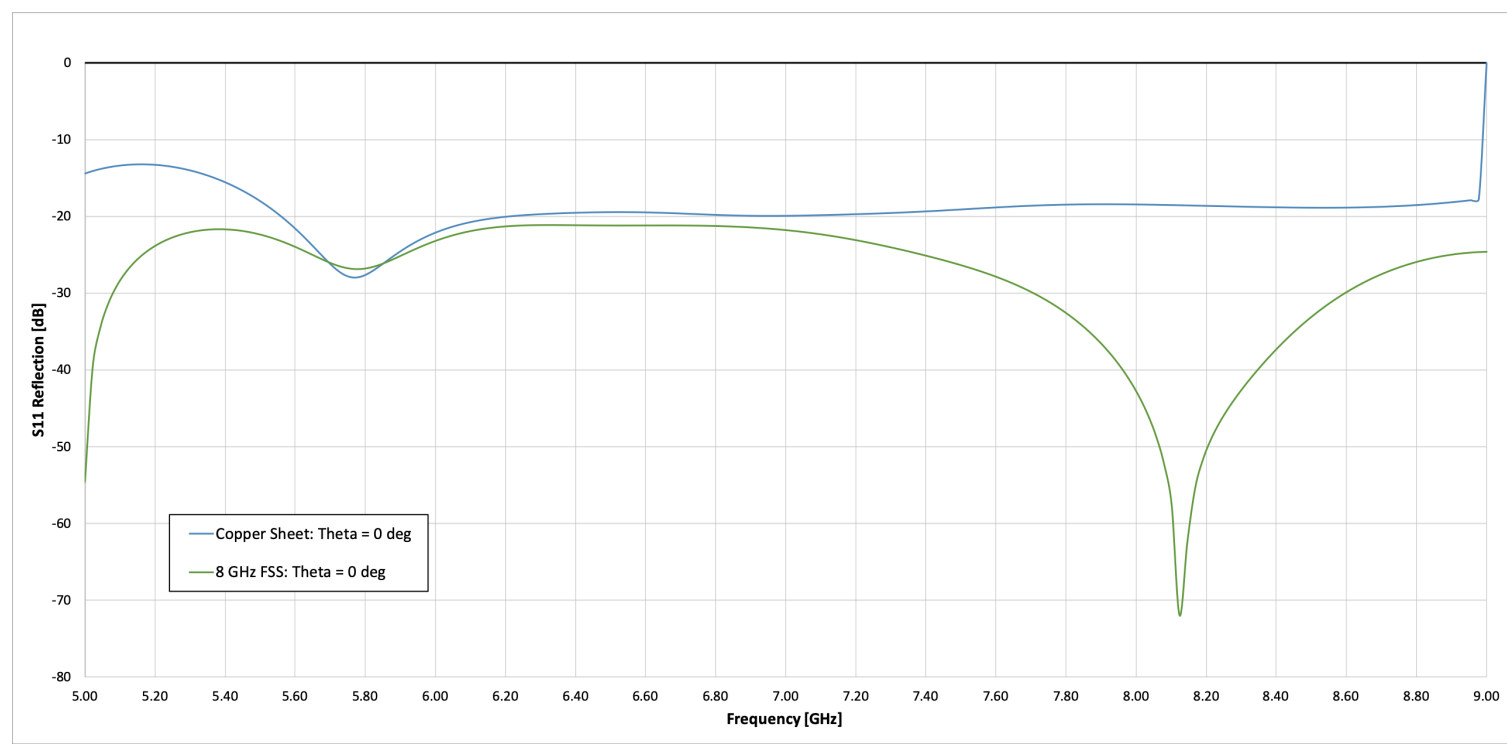

Figure 5.7: Measured $S_{11}$ response vs. frequency, copper sheet (blue), $8 \mathrm{GHz}$ FSS (green), TDR gating distance $0.4809 \mathrm{~m}-0.6581 \mathrm{~m}$.

Figure 5.8 below shows the $8 \mathrm{GHz}$ FSS reflection measurements for incident angles $0^{\circ}-45^{\circ}$ in $15^{\circ}$ steps with no TDR gating. Significant differences are not discernible. Therefore, the same TDR gating technique is used. Figure 5.9 shows the $8 \mathrm{GHz}$ FSS $S_{11}$ reflection measurements for incident angles $0^{\circ}-45^{\circ}$ in $15^{\circ}$ steps with TDR gating. The TDR gating passband is slightly modified for each measurement due to changes in physical distance between the angled FSS and the horn antenna.

Figure 5.9 shows resonant frequency shifts are minimal with incident angle variation. Theta $=15^{\circ}$ shows greatest resonant frequency shift to $f_{r}=7.90 \mathrm{GHz}$ with $\Delta f=-0.22 \mathrm{GHz}$. However, simulation in Figure 4.17 shows a similar frequency deviation at theta $=15^{\circ}$. 


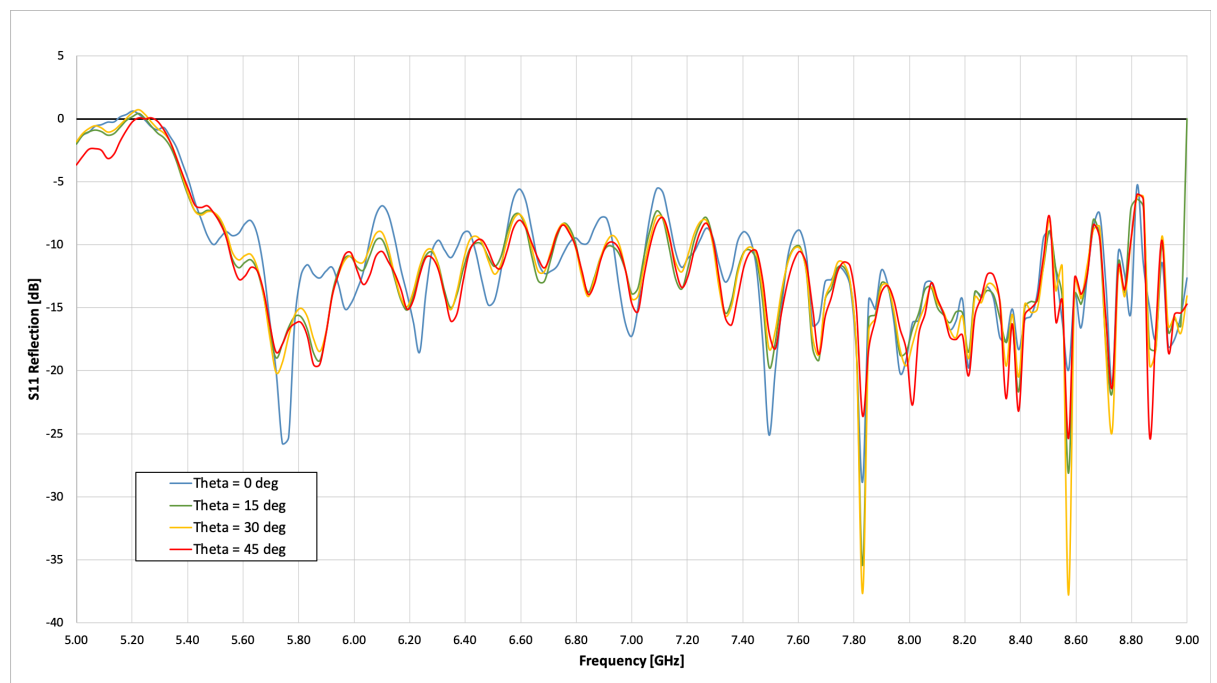

Figure 5.8: Measured $S_{11}$ response vs. frequency, Theta $=0^{\circ}-45^{\circ}$, no TDR gating.

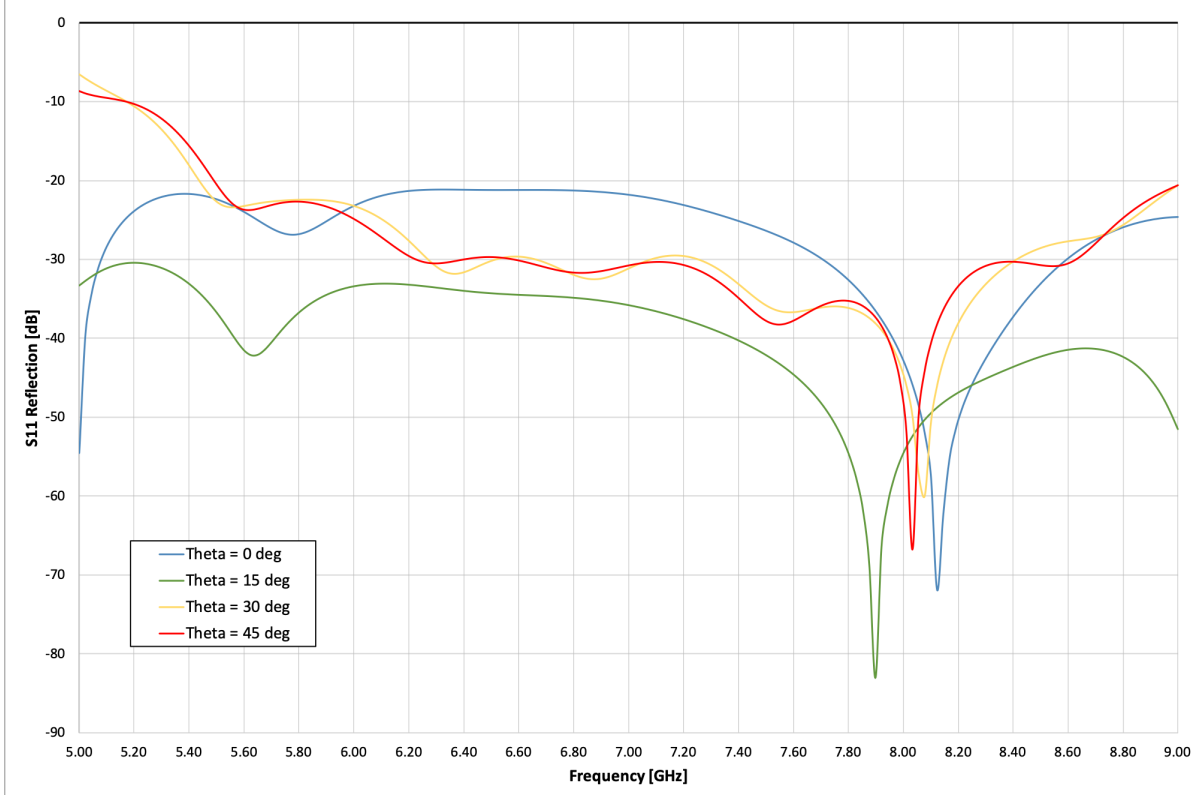

Figure 5.9: Measured $S_{11}$ response vs. frequency, Theta $=0^{\circ}-45^{\circ}$, TDR gating used: $45^{\circ}=0.252 m-0.5928 m, 30^{\circ}=0.252 m-0.6015 m, 15^{\circ}=$ $0.5497 m-0.6279 m, 0^{\circ}=0.4809 m-0.6581 m$.

\subsubsection{Transmission Characteristics}

Figures 5.10 and 5.11 below shows the $10 \mathrm{~cm} \times 10 \mathrm{~cm}$ copper sheet compared to no sheet, and $8 \mathrm{GHz}$ FSS compared to no sheet measured $S_{21}$ response, respectively. The 
$8 \mathrm{GHz}$ FSS $S_{21}$ shows significant attenuation for $f=5-7 G H z$ range. The FSS response also more closely matches the no sheet response for $f=7.8-9 G H z$ range. The FSS attenuates transmissions outside the resonant frequency range $f<7.8 \mathrm{GHz}$.

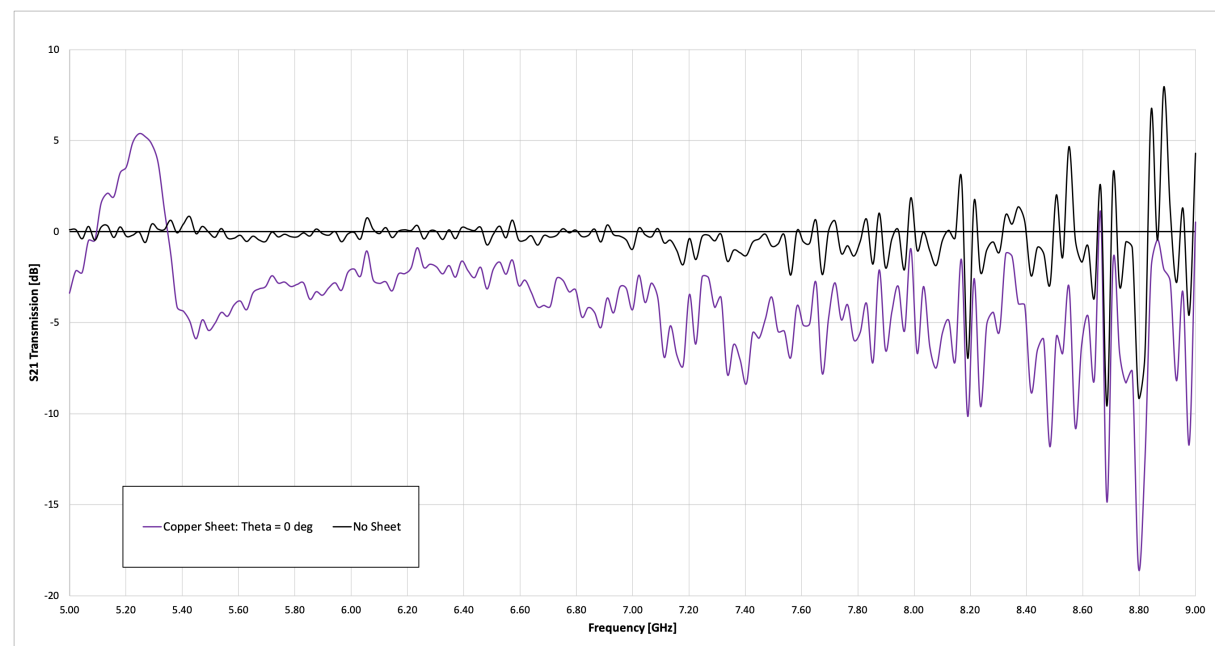

Figure 5.10: $10 \mathrm{~cm}$ x $10 \mathrm{~cm}$ Copper sheet, and No Sheet measured $S_{21}$ vs. frequency.

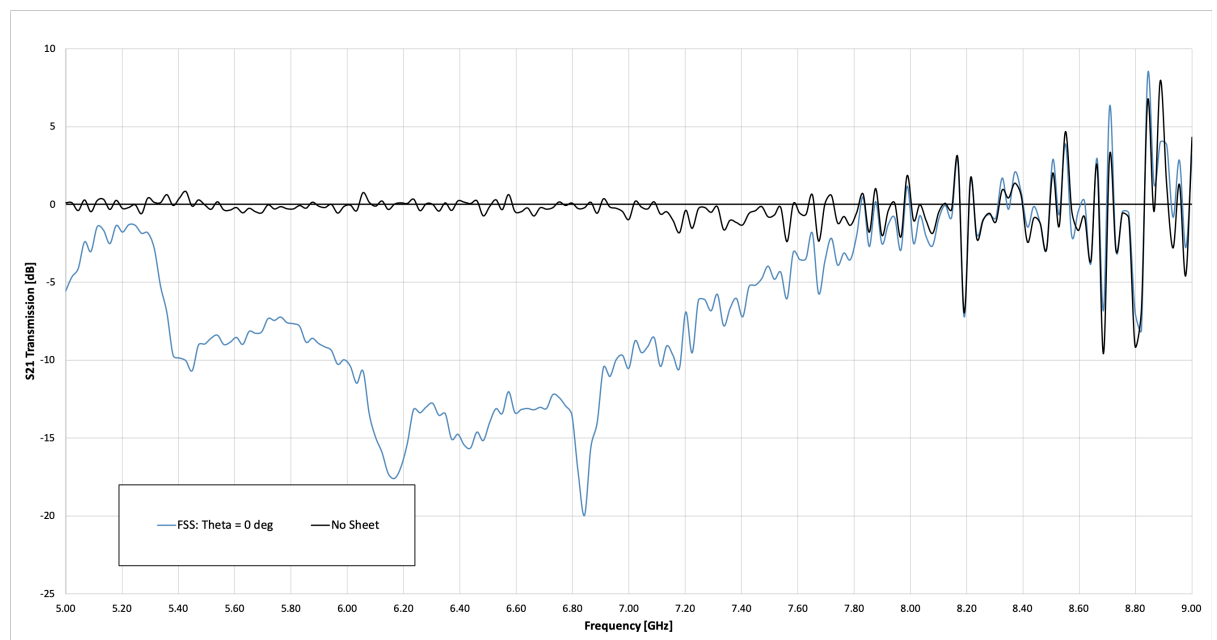

Figure 5.11: $8 \mathrm{GHz}$ FSS, and No Sheet measured $S_{21}$ vs. frequency.

The $8 \mathrm{GHz}$ FSS passband is depicted as $\tau_{1}$ in Figure 5.4. To isolate this measurement and show the calculated FSS passband transmission response, $\tau_{2}$ is calculated and factored out of the $S_{21}$ measurement. The $8 \mathrm{GHz}$ FSS passband is calculated for frequency range $f=7.60-9 \mathrm{GHz}$. Figure 5.12 below shows the calculated $8 \mathrm{GHz}$ 


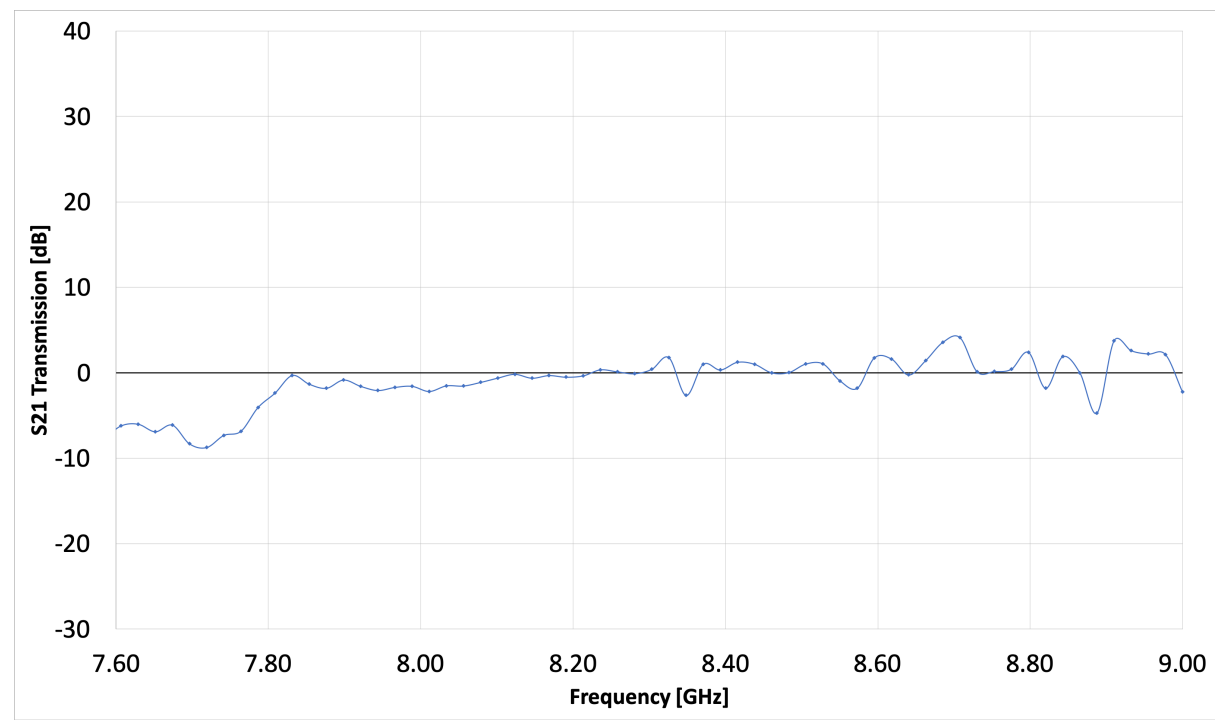

Figure 5.12: Calculated $S_{21}$ passband, 8 GHz FSS, $f=7.60-9 G H z$

passband. The response maximal transmission in this frequency range. Indicating the $8 \mathrm{GHz}$ FSS is transparent to the propagating EM waves in this frequency range.

Figure 5.13 shows the $8 \mathrm{GHz}$ FSS measured $S_{21}$ at various theta angles. Transmission characteristics show similar performance for each incident angle in the antenna operating range: $7.05 \mathrm{GHz}-10 \mathrm{GHz}$.

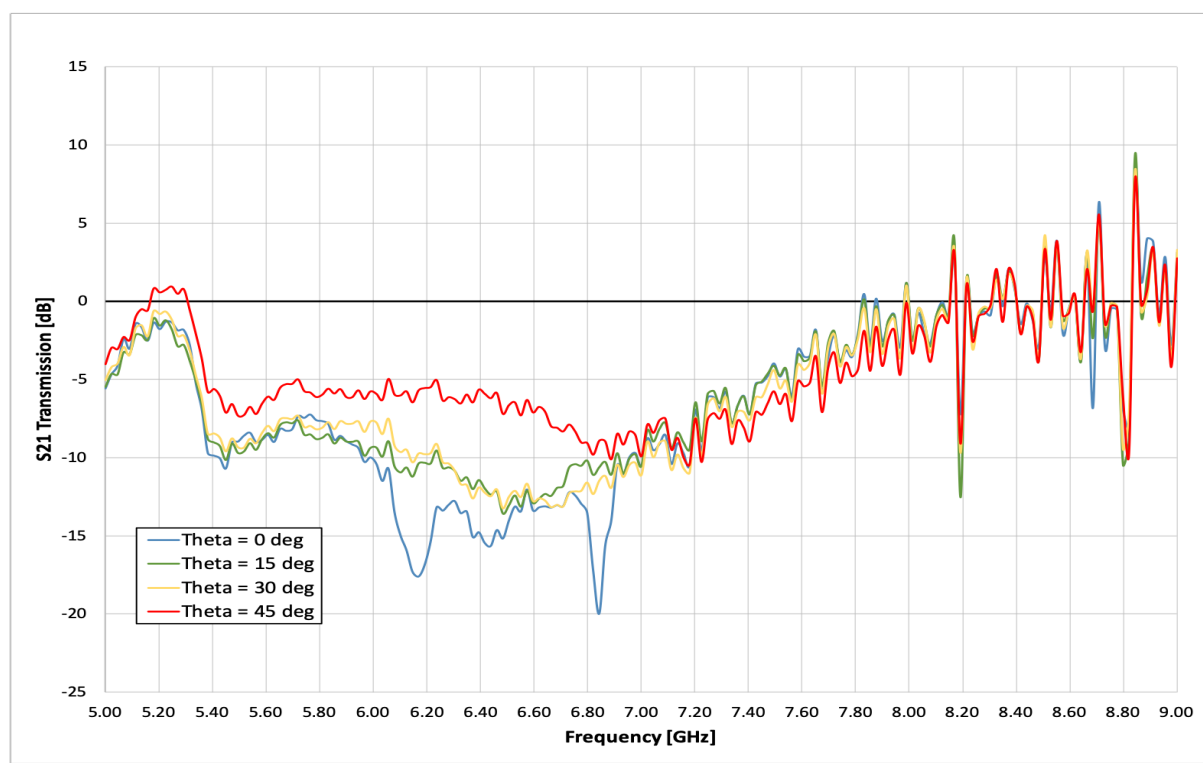

Figure 5.13: $8 \mathrm{GHz}$ FSS measured $S_{21}$ vs. frequency at various theta angles. 


\section{$5.279 \mathrm{GHz}$ FSS}

\subsection{1 mmWave Methodology}

The 79 GHz FSS's performance is tested using the IWR1642 radar and DCA1000EVM. TI provides a software user interface, mmWave Studio, hat enables IWR1642 ADC data analysis. A 1-D FFT is applied to the measured ADC data to determine received power peaks vs. distance from the radar. This data is used to determine the test object's reflective properties. Figure 5.14 below shows the IWR1642 and DCA1000 experimental setup.

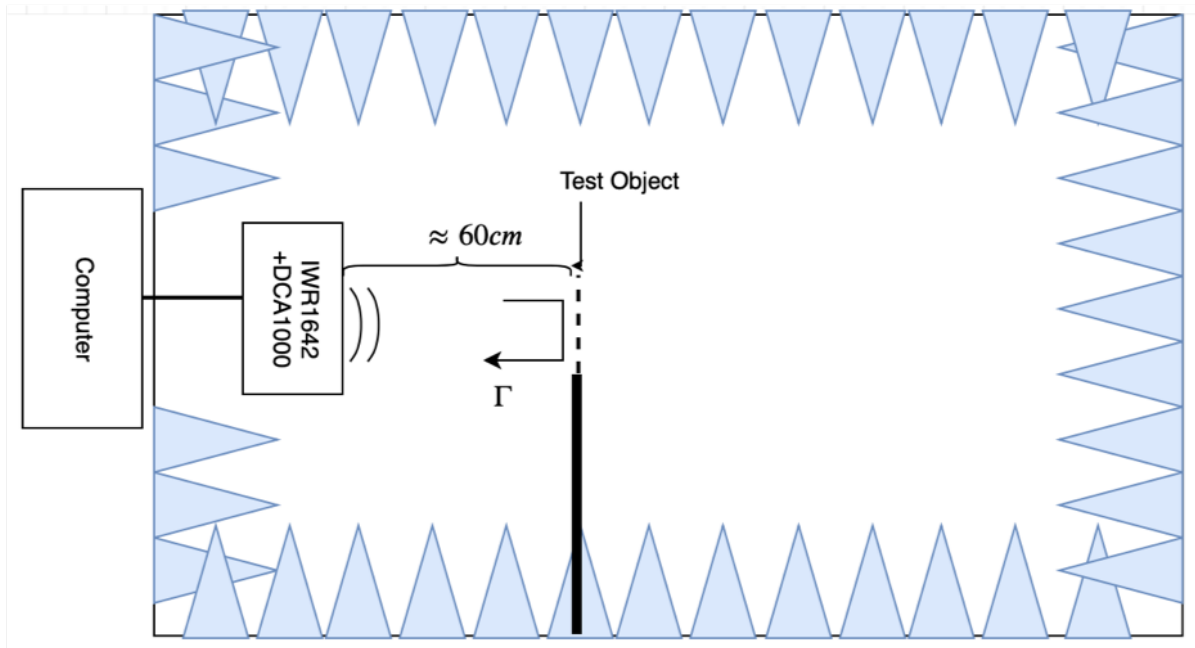

Figure 5.14: IWR1642 and DCA1000 experimental setup.

\subsubsection{Reflection Characteristics}

The amplitude measurements for the $79 \mathrm{GHz}$ FSS and the $6 \mathrm{~cm} \times 6 \mathrm{~cm}$ copper sheet are show in Figures 5.15 and 5.16, respectively. Table 5.1 hows the ADC measured amplitudes recorded by the IWR1642 and DCA1000. The peak amplitude is measured in decibels with respect to full scale (dBFS). The $6 \mathrm{~cm} \times 6 \mathrm{~cm}$ copper sheet is a blank copper PCB cut to the same physical cross section as the $79 \mathrm{GHz}$ FSS. The $10 \mathrm{~cm} \times 10 \mathrm{~cm}$ copper sheet is the same copper sheet used in the $8 \mathrm{GHz}$ testing. 


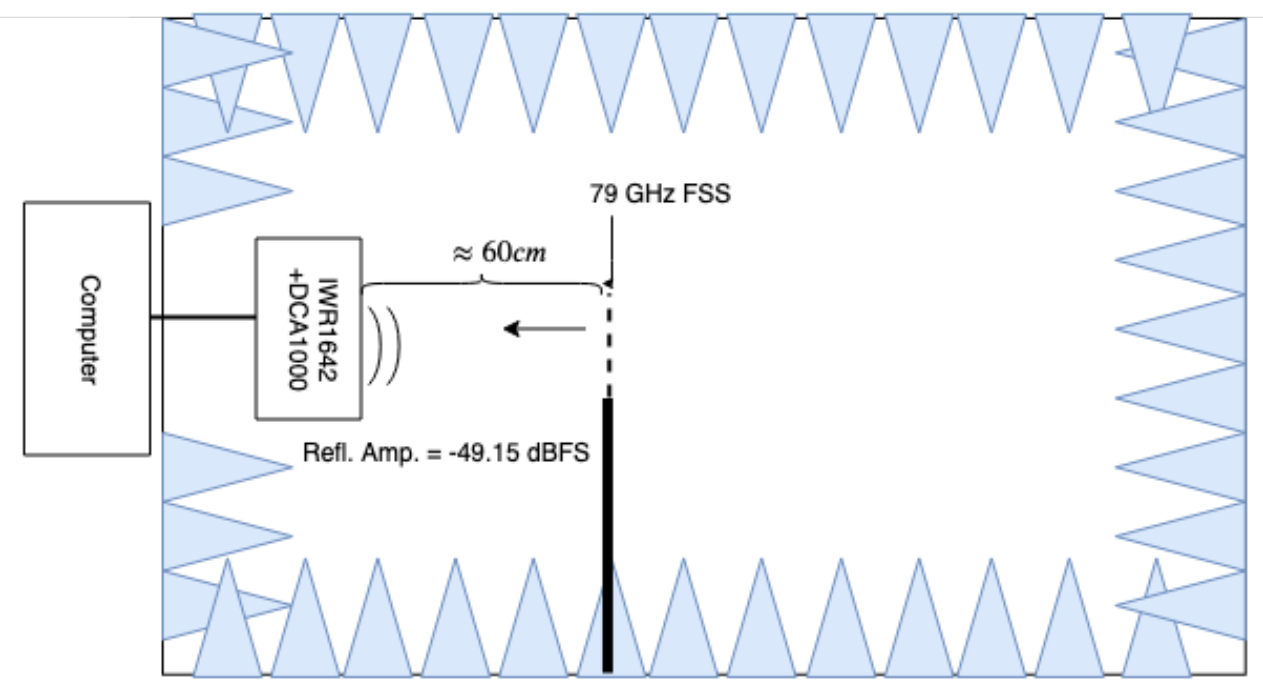

Figure 5.15: Measured Reflection diagram, $79 \mathrm{GHz}$ FSS.

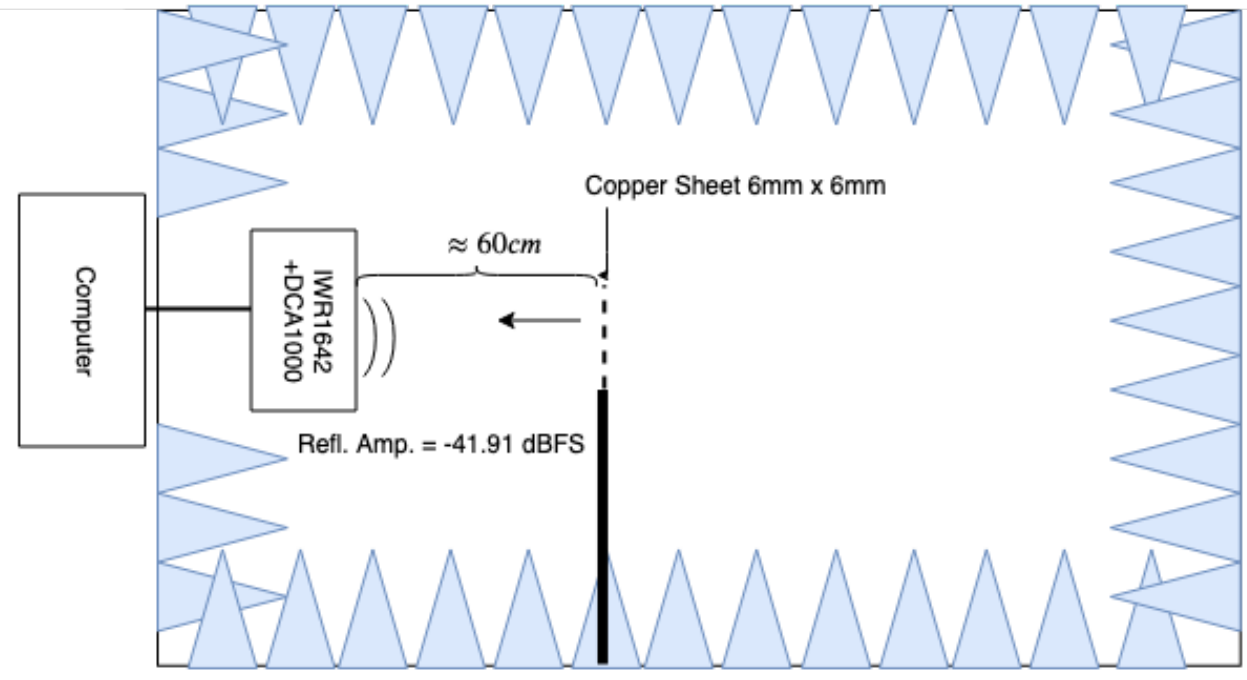

Figure 5.16: Measured Reflection diagram, $6 \mathrm{~cm}$ x $6 \mathrm{~cm}$ copper sheet.

The measured $79 \mathrm{GHz}$ FSS amplitude shows a $-7.24 \mathrm{~dB}$ reduction in amplitude compared to the $6 \mathrm{~cm} \times 6 \mathrm{~cm}$ copper sheet. This indicates an increase in transmitted power through the $79 \mathrm{GHz}$ FSS at the radar frequency range. This is compared to $\mathrm{a}+0.4 \mathrm{~dB}$ difference between the $8 \mathrm{GHz}$ FSS and the $10 \mathrm{~cm}$ x $10 \mathrm{~cm}$ copper sheet. 
Table 5.1: 1D FFT amplitude peaks for various surfaces at $58.59 \mathrm{~cm}$ from the radar

\begin{tabular}{|c|c|}
\hline Surface & Peak Amplitude [dBFS] \\
\hline $79 \mathrm{GHz}$ FSS & -49.15 \\
\hline $6 \mathrm{~cm} \times 6 \mathrm{~cm}$ Copper Sheet & -41.91 \\
\hline $8 \mathrm{GHz}$ FSS & -36.83 \\
\hline $10 \mathrm{~cm} \times 10 \mathrm{~cm}$ Copper Sheet & -37.23 \\
\hline No Surface & -65.82 \\
\hline
\end{tabular}

The similar response in the $8 \mathrm{GHz}$ FSS and $10 \mathrm{~cm}$ x $10 \mathrm{~cm}$ copper sheet indicates the $8 \mathrm{GHz}$ FSS does not resonate at the radar chirp frequency range, and it reflects the plane waves similar to the $10 \mathrm{~cm}$ x $10 \mathrm{~cm}$ copper sheet. 
Chapter 6

CONCLUSIONS

This work examines FSS applications to object visibility in mmWave radar. Loaded cross aperture FSS screens are designed and fabricated at $8 \mathrm{GHz}$ and $79 \mathrm{GHz}$. The $8 \mathrm{GHz}$ FSS reflection and transmission characteristics are examined and demonstrate filtering properties in agreement with HFSS simulations. Table 6.1 below shows the $8 \mathrm{GHz}$ FSS simulated and measured first resonance frequency. Minimal deviation from simulated results indicates this HFSS simulation method is appropriate for this design and the 2-D infinite sheet approximation is verified. The downward shift in resonance frequency at $15^{\circ}$ incident angle is demonstrated in simulations and measured data.

Table 6.1: Simulated and measured resonance frequency at various angles, $8 \mathrm{GHz}$ FSS

\begin{tabular}{|c|c|c|c|}
\hline Incident Angle & Simulated $f_{r}$ & Measured $f_{r}$ & Difference $\Delta f$ \\
\hline $0^{\circ}$ & $8.07 \mathrm{GHz}$ & $8.12 \mathrm{GHz}$ & $0.05 \mathrm{GHz}$ \\
\hline $15^{\circ}$ & $7.26 \mathrm{GHz}$ & $7.90 \mathrm{GHz}$ & $0.64 \mathrm{GHz}$ \\
\hline $30^{\circ}$ & $8.10 \mathrm{GHz}$ & $8.08 \mathrm{GHz}$ & $0.02 \mathrm{GHz}$ \\
\hline $45^{\circ}$ & $8.11 \mathrm{GHz}$ & $8.03 \mathrm{GHz}$ & $0.08 \mathrm{GHz}$ \\
\hline
\end{tabular}

This FSS design demonstrates minimal resonant frequency shift when incident angle is varied. Therefore, the loaded cross aperture configuration is desirable in scenarios where incident angle is highly variable. This is the case in autonomous vehicles where radars and the objects around them are in motion. The $79 \mathrm{GHz}$ FSS reflection measurements demonstrate minimal reflections in the IWR1642 radar's frequency range when compared to the $6 \mathrm{~cm}$ x $6 \mathrm{~cm}$ copper sheet. Indicating the $79 \mathrm{GHz}$ FSS is resonating in the radar's chirp frequency range $(77 \mathrm{GHz}-81 \mathrm{GHz})$. However, tolerance simulations show that mmWave frequency FSSs require precision manufacturing to the micron scale. Therefore, standard PCB fabrication methods 
may not be suitable to mass production of these devices. Enhanced precision copper etching manufacturing techniques is one possible method of fabricating FSSs. 


\section{Chapter 7}

\section{FUTURE WORK}

This research has further proven that HFSS is an effective design tool for modeling and simulating FSSs. Results demonstrate confidence that the 2D infinite plane approximation is appropriate in FSS design. Further research is necessary reduce the precision required to manufacture FSSs without compromising performance. The next steps are to refine the concept of creating highly visibly objects for $77 \mathrm{GHz}$ radar. Frequency selective optical reflectors have been produced [13]. Radar corner cubes are used in practice today to create highly reflective objects. Applying a frequency selective surface to this structure would impart frequency selective properties to the corner cube. Future research in construction of a low cost and discrete, frequency selective reflector in the mmWave frequency range is the next phase of this research. 


\section{BIBLIOGRAPHY}

[1] Ansys. Getting started with hfss: Floquet ports. http://www.1cae.com/ articleAccessory/2017/04/16/HFSS\%20Floquet\%20Ports.pdf, 72016.

[2] G. hui Yang, S. Ma, E. chao Wang, Y. Qin, and X. Gu. Design of active frequency selective surfaces for the rcs reduction. International Journal of Security and Its Applications, 7:407-414, 092013.

[3] T. Instruments. Ti mmwave sensors awr1x family device overview. https://training.ti.com/sites/default/files/docs/TI\%20mmWave\% 20device\%20overview_AWR1x.pdf.

[4] T. Instruments. Awr1642 evaluation module (awr1642boost) single-chip mmwave sensing solution. http://www.ti.com/lit/ug/swru508b/swru508b.pdf, 4 2018. User's Guide.

[5] A. Itou, O. Hashimoto, H. Yokokawa, and K. Sumi. A fundamental study of a thin $\frac{\lambda}{4}$ wave absorber using fss technology. Electronics and Communications in Japan (Part I: Communications), 87:77 - 86, 112004.

[6] C. Lovescu and S. Rao. The fundamentals of millimeter wave sensors. http://www.ti.com/lit/wp/spyy005/spyy005.pdf, 52017.

[7] M. Lynberg. Automated vehicles for safety. https://www.nhtsa.gov/technology-innovation/automatedvehicles-safety, 2018. Accessed: 29-April-2019.

[8] B. A. Munk. Frequency Selective Surfaces, Theory and Design. John Wiley, 2000. 
[9] S. Narayan, B. Sangeetha, and R. Mohan Jha. Frequency Selective Surfaces based High Performance Microstrip Antenna. Springer, 2016.

[10] F. Sakran, Y. Neve-Oz, A. Ron, M. Golosovsky, D. Davidov, and A. Frenkel. Absorbing frequency-selective-surface for the mm-wave range. IEEE Transactions on Antennas and Propagation, 56(8):2649-2655, Aug 2008.

[11] B. Schoenlinner, J. P. Ebling, L. C. Kempel, and G. M. Rebeiz. Compact multibeam dual-frequency (24 and $77 \mathrm{ghz}$ ) imaging antenna for automotive radars. In 2003 33rd European Microwave Conference, pages 785-788, Oct 2003.

[12] R. D. Seager, A. Chauraya, J. Bowman, M. Broughton, and N. Nimkulrat. Fabrication of fabric based frequency selective surfaces (fss). In The 8th European Conference on Antennas and Propagation (EuCAP 2014), pages 1978-1980, April 2014.

[13] R. J. Williams. FREQUENCY SELECTIVE TERAHERTZ RETROREFLECTORS. Master's thesis, Universsity of Massachusetts Lowell, 2014.

[14] T.-K. Wu. Frequency-selective surfaces. In Encyclopedia of RF and Microwave Engineering, pages 1017-1023. John Wiley, 042005. 